\title{
Variedades determinantais e singularidades de matrizes
}

\author{
Miriam da Silva Pereira ${ }^{1}$
}

Orientadora: Profa. Dra. Maria Aparecida Soares Ruas

Tese apresentada ao Instituto de Ciências Matemáticas e de Computação - ICMC-USP, como parte dos requisitos para obtenção do título de Doutor em Ciências - Matemática.

\section{USP - São Carlos}

Abril/2010

\footnotetext{
${ }^{1}$ Suporte FAPESP Processo: 05/58960-3
} 

Variedades determinantais e singularidades de matrizes

Miriam da Silva Pereira 

"É melhor tentar e falhar, que preocupar-se e ver a vida passar. É melhor tentar, ainda que em vão que sentar-se, fazendo nada até o final. Eu prefiro na chuva caminhar, que em dias frios em casa me esconder. Prefiro ser feliz embora louco, que em conformidade viver". 

À minha mãe,

Celia.

dedico. 



\section{Agradecimentos}

Ao término deste trabalho, gostaria de à agradecer Deus, pelo precioso dom da vida e por cumprir, a cada dia, a promessa: "Mas os que esperam no Senhor renovam as suas forças, sobem com asas como águias, correm e não se cansam, caminham e não se fadigam (Isaías 40:31).

A realização desse projeto se tornou mais agradável porque tive o privilégio de conhecer pessoas especiais para as quais preciso expressar minha gratidão.

À minha mãe Celia, por toda sua compreensão, apoio, dedicação e paciência durante esta longa caminhada.

Aos meus irmãos, Davi e Katia, pelo companheirismo de uma vida.

Á toda minha família, tios, primos e amigos pela confiança. A vida se torna muito melhor com todas as histórias, conversas e almoços nos fins de semana.

À Professora Maria Aparecida Soares Ruas por ter me orientado durante este período. Com ela, tive a oportunidade de aprender muita coisa, tanto do ponto de vista matemático, como do ponto de vista humano. Poucas pessoas conseguem unir qualidades tão especiais como ela.

Aos professores Jan Stevens, Osamu Saeki e Terence Gaffney pelas importantes sugestões que foram fundamentais para a realização deste deste trabalho.

As minhas companheiras de estudos, aventuras e viagens Grazielle, Marcela e Michelle. Amigas como vocês não se encontram em qualquer van, consulado, hospedaria antiga ou Ciudadela por aí! Meninas, lembrem-se: Vocês poderão ir onde quiserem!

Ao Nivaldo, por toda a paciência e precisão em responder minha "listinha"interminável de perguntas. Preciso deixar registrado que você é uma das pessoas mais educadas e inteligentes que já conheci!

Ao Marcus (Marcio!), por todo companheirismo durante as disciplinas do primeiro ano. Aprendi muito com seus desenhos!

Aos colegas de pós- graduação pelo convívio e aos professores e funcionários do ICMC- USP, por toda a disponibilidade em ajudar sempre que necessário.

À Capes e a FAPESP² pelo auxílio financeiro.

\footnotetext{
${ }^{2}$ Processo Fapesp: 05/58960-3
} 



\section{Resumo}

O teorema de Hilbert-Burch fornece uma boa descrição de variedades determinantais de codimensão dois e de suas deformações em termos da matriz de representação. Neste trabalho, usamos esta correspondência para estudar propriedades de tais variedades usando métodos da teoria de singularidades. Na primeira parte da tese, estabelecemos a teoria de singularidades de matrizes $n \times p$, generalizando os resultados obtidos por J. W. Bruce and F. Tari em [5], para matrizes quadradas, e por A. Frühbis-Krüger em [16], para matrizes $n \times(n+1)$. Na segunda parte, nos concentramos em variedades determinantais de codimensão 2 , com singularidade isolada na origem. Para estas variedades, podemos mostrar a existência e a unicidade de suavizações, o que possibilita definir seu número de Milnor como o número de Betti na dimensão média de sua fibra genérica. Para superfícies em $\mathbb{C}^{4}$, obtemos uma fórmula Lê-Greuel expressando o número de Milnor da superfície em termos da segunda multiplicidade polar e do número de Milnor de uma seção genérica. 


\begin{abstract}
The theorem of Hilbert- Burch provides a good description of codimension two determinantal varieties and their deformations in terms of their presentation matrices. In this work we use this correspondence to study properties of determinantal varieties, based on methods of singularity theory of their presentation matrices. In the first part of the thesis we establish the theory of singularities for $n \times p$ matrices extending previous results of J. W. Bruce and F. Tari in [5], for classes of square matrices, and A. Frühbis-Krüger for $n \times(n+1)$ matrices in [16]. In the second part we concentrate on codimension two determinantal varieties with isolated singularities. These singularities admit a unique smoothing, thus we can define their Milnor number as the middle Betti number of their generic fiber. For surfaces in $\mathbb{C}^{4}$, we obtain a Lê-Greuel formula expressing the Milnor number of the surface in terms of the second polar multiplicity and the Milnor number of the generic section.
\end{abstract}




\section{Sumário}

1 Definições e Resultados Básicos $\quad 1$

1.1 Variedades Analíticas . . . . . . . . . . . . . . . . . . . . 1

1.2 Teoria de Morse . . . . . . . . . . . . . . . . . . . . . . . 4

1.3 Multiplicidade Polar . . . . . . . . . . . . . . . . . . . . . . 5

1.4 Anéis Cohen-Macaulay . . . . . . . . . . . . . . . . . . . . 7

1.5 Ideais Determinantais . . . . . . . . . . . . . . . . . . . . 10

2 Teoria de Singularidades de Matrizes $\quad 14$

2.1 Notações . . . . . . . . . . . . . . . . . . . . . . . . . . . 14

2.2 O $\mathcal{G}$ - Espaço Tangente . . . . . . . . . . . . . . . . . . . . 15

2.3 Determinação Finita . . . . . . . . . . . . . . . . . . . . 16

2.4 Determinação Finita: Critério Geométrico . . . . . . . . . . . . . . . 23

2.5 Subgrupos Geométricos . . . . . . . . . . . . . . . . . 25

2.6 Propriedades da $\mathcal{G}$ - Equivalência . . . . . . . . . . . . . . . . . . 30

3 G- Equivalência Topológica de Matrizes $\quad 41$

3.1 Um Teorema de Trivialidade Topológica . . . . . . . . . . . . . . . . . . 41

3.2 Deformações de Germes Quase- Homogêneneos . . . . . . . . . . . . . . . . . . . 44

4 Deformações de germes Cohen-Macaulay de Codimensão $2 \quad 51$

4.1 Deformações Infinitesimais . . . . . . . . . . . . . . . . . . . . . . 51

4.2 Deformações de Germes Cohen-Macaulay de Codimensão 2 . . . . . . . . . . 53

$4.3 \mathcal{G}$-Estabilidade de Matrizes . . . . . . . . . . . . . . . . . . . 56

$\begin{array}{lll}5 & \text { A Fibra de Milnor } & 60\end{array}$

5.1 Suavizações e Bola de Milnor . . . . . . . . . . . . . . . . . . . 60

5.2 A Topologia de Variedades com Singularidade Isolada . . . . . . . . . . . . . . . 63 


\section{Invariantes de Variedades Determinantais}

6.1 Variedades Determinantais . . . . . . . . . . . . . . . 67

6.2 Índice de 1-Formas em Variedades Determinantais . . . . . . . . . . . . . . 69

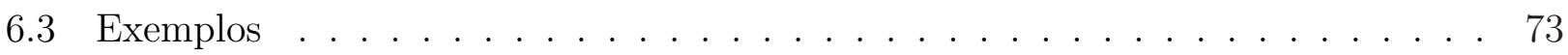

Referências Bibliográficas $\quad 83$ 


\section{Introdução}

Sejam $R$ um anel comutativo, $M$ uma matriz $n \times p$ com entradas em $R$ e $t \leq \min \{n, p\}$. Um ideal $I \subset R$ é chamado determinantal se é gerado pelos menores $t \times t$ da matriz $M$. Se $X$ é uma variedade dos zeros de um ideal determinantal, então dizemos que $X$ é uma variedade determinantal. Os ideais de definição de algumas variedades clássicas que aparecem em geometria algébrica, tais como as variedades determinadas pelos mergulhos de Segre e de Veronese, são determinantais. Intersecções completas também são variedades determinantais com $p=t=1$.

Uma classe importante de variedades determinantais é obtida quando as entradas da matriz $M$ são indeterminadas sobre um corpo.

As propriedades de variedades determinantais têm sido amplamente estudadas tanto pela álgebra comutativa quanto pela geometria algébrica (ver [7], [8]). Se $M$ é uma matriz com entradas em $R$ e $t=\min \{n, p\}$, J. Eagon mostra em [12] que o ideal $I$ gerado pelos menores $t \times t$ de $M$ é Cohen-Macaulay. Posteriormente, este resultado foi generalizado por J. Eagon e Hochester em [27] para matrizes cujas entradas são indeterminadas sobre um corpo e $t$ é arbitrário.

Em particular, no caso em que $X$ é uma variedade determinantal de codimensão dois, podemos usar o Teorema de Hilbert-Burch para obter uma boa descrição de $X$ e de suas deformações em termos de sua matriz de representação. Isto é, se $X$ é uma variedade CohenMacaulay de codimensão dois, então $X$ pode ser definida pelos menores maximais de uma matriz $n \times(n+1)$. Além disso, qualquer deformação de uma matriz $n \times(n+1)$ dá origem a uma deformação de $X$ e qualquer deformação de $X$ pode ser obtida através de uma perturbação da matriz de representação.

Neste trabalho, usamos esta correspondência para estudar propriedades de variedades que são Cohen-Macaulay de codimensão 2 através de sua matriz de representação. Podemos expressar a operação do grupo de contato em termos matriciais e definir um grupo $\mathcal{G}$ que age no espaço das matrizes, através de mudanças de coordenadas na fonte e multiplicação à esquerda e 
à direita por matrizes invertíveis. Desta forma, podemos usar esta equivalência para classificar germes de matrizes.

Em [16], A. Frühbis-Krüger considera o caso de matrizes que definem singularidades que são Cohen- Macaulay de codimensão 2 com o objetivo de estudar curvas espaciais. Neste caso, é possível obter o módulo normal e o espaço $T^{1}$ das deformações de primeira ordem em termos da matriz de representação. O número de Tjurina, $\tau(X)$, da variedade determinantal $X$ é a codimensão do espaço $T^{1}$ e coincide com a $\mathcal{G}_{e^{-}}$codimensão de $M$. Em [18], A. Frühbis-Krüger e A. Neumer obtêm uma classificação completa dos germes simples de singularidades que são Cohen-Macaulay de Codimensão 2.

Matrizes quadráticas já foram estudadas anteriormente por V. I. Arnold em [2]. Além disso, usando a $\mathcal{G}$-equivalência de matrizes, J. W. Bruce, F. Tari e G. J. Haslinger obtêm classificações de germes simples de famílias de matrizes simétricas, antisimétricas e quadradas usando a $\mathcal{G}$ equivalência ([3], [5], [28]).

Estabelecemos, na primeira parte do trabalho, a teoria de singularidades para matrizes $n \times p$, estendendo os resultados de [16], [3] e [4] referentes à caracterização infinitesimal e geométrica da determinação finita e o teorema de desdobramento versal.

Um resultado interessante do trabalho é a descrição de condições equivalentes à $\mathcal{G}$ - determinação finita da matriz de representação de variedades Cohen-Macaulay de codimensão 2 com singularidade isolada. O resultado correspondente para o grupo de contato $\mathcal{K}$ foi obtido por $\mathrm{T}$. Gaffney em [20].

Os principais resultados deste trabalho são obtidos para germes de matrizes que definem variedades determinantais $X$ de codimensão 2 com singularidade isolada. Nesta classe, podemos mostrar a existência e a unicidade de suavizações. Se $X$ tem dimensão $d$, é possível definir o número de Milnor de $X, \mu(X)$, como sendo o $d$ - ésimo número de Betti da fibra genérica de uma suavização de $X$. No caso em que $X$ é uma superfície determinantal em $\mathbb{C}^{4}$ com singularidade isolada na origem, obtemos uma fórmula do tipo Lê- Greuel que expressa o número de Milnor de $X$ em termos da segunda multiplicidade polar e do número de Milnor de uma seção genérica de $X$.

Relacionamos também o índice de 1- formas em variedades determinantais definido por W. Ebeling e S. M. Gusein-Zade em [13], com a multiplicidade polar e, consequentemente, com o número de Milnor de $X$. Como aplicação dos resultados obtidos, calculamos o número de Milnor para algumas formas normais da classificação de superfícies simples Cohen-Macaulay de codimensão 2 obtida em [18].

No Capítulo 1, relembramos algumas definições e resultados básicos tais como germes de 
espaços analíticos complexos e funções analíticas com singularidade isolada em relação a uma estratificação destes espaços. Além disso, apresentamos os conceitos e propriedades de anéis Cohen-Macaulay e de variedades determinantais.

No Capítulo 2, introduzimos a $\mathcal{G}$ - equivalência de matrizes, definimos o espaço tangente e estudamos a $\mathcal{G}$ - determinação finita. Além disso, mostramos que $\mathcal{G}$ é um subgrupo geométrico, conforme definição dada por J. Damon em [10]. Para esta classe de subgrupos do grupo de contato, os métodos usuais da teoria de singularidades se aplicam e os teoremas de caracterização de determinação finita e desdobramento versal se verificam. As singularidades CohenMacaulay de codimensão 2 isoladas são também estudadas e várias condições equivalentes à $\mathcal{G}$-determinação finita são obtidas na seção final do capítulo.

Como aplicação dos resultados do Capítulo 2, estudamos, no Capítulo 3, a $\mathcal{G}$ - trivialidade topológica de famílias de matrizes.

No capítulo 4, definimos a noção de deformação de um germe analítico complexo $X$ e estudamos, em particular, deformações de germes Cohen-Macaulay de codimensão 2.

O Capítulo 5, contém alguns resultados sobre a topologia de variedades analíticas complexas com singularidade isolada, tais como a definição de bola de Milnor e de suavização. Além disso, usamos a Teoria de Morse para descrever o tipo de homotopia da fibra genérica da suavização de variedades Cohen-Macaulay de codimensão 2.

No capítulo 6, definimos o conceito de número de Milnor para variedades Cohen-Macaulay de codimensão 2, com singularidade isolada na origem. O resultado principal deste capítulo é o teorema que estabelece uma fórmula do tipo Lê-Greuel para superfícies determinantais em $\mathbb{C}^{4}$. Além disso, relacionamos o índice de 1-forma em variedades determinantais de [13] com a 2multiplicidade polar de $X$. Utilizando os resultados obtidos nos capítulos anteriores, calculamos o número de Milnor para algumas das superfícies simples classificadas em [18]. 


\section{Capítulo 1}

\section{Definições e Resultados Básicos}

Neste capítulo vamos relembrar algumas definições e resultados clássicos necessários ao desenvolvimento desta tese. Iniciamos introduzindo germes de espaços analíticos complexos e de funções analíticas com singularidade isolada em relação a uma estratificação destes espaços. Posteriormente, estudamos os conceitos de anéis Cohen-Macaulay e de variedades determinantais.

As principais referências para este capítulo são [8], [15], [1], [49] e [30].

\subsection{Variedades Analíticas}

Introduzimos nesta seção alguns conceitos fundamentais da teoria de singularidades e de funções definidas em variedades.

Seja $\mathbb{K}$ o corpo dos números reais ou complexos. Indicamos por $\mathcal{O}_{r}$ o anel local dos germes de funções analíticas de $\mathbb{C}^{r}$ na origem e $\mathfrak{M}$ seu ideal maximal. Além disso, seja $\mathcal{E}_{r}$ o anel dos germes de funções $C^{\infty}$ na origem. Usaremos a notação $\mathcal{P}$ para representar qualquer um destes anéis. Seja $\mathbb{K}\left[x_{1}, \ldots, x_{r}\right]$ o anel dos polinômios em $r$ variáveis sobre $\mathbb{K}$.

Definição 1.1.1. Seja $Y \subset \mathbb{C}^{r}$. Se existe um subconjunto $T \subset \mathbb{C}\left[x_{1}, \ldots, x_{r}\right]$ tal que $Y$ é o conjunto de zeros comuns a todos os polinômios de $T$, dizemos que $Y$ é um conjunto algébrico.

É possível mostrar que a união e a intersecção de conjuntos algébricos é ainda um conjunto algébrico. Os conjuntos algébricos são os fechados da topologia de Zariski de $\mathbb{C}^{r}$ (ver [30]).

Definição 1.1.2. Dizemos que um subconjunto não vazio $Y \subset X$ é irredutível se o mesmo não pode ser escrito como uma união $Y=Y_{1} \cup Y_{2}$, onde $Y_{1}, Y_{2}$ são fechados em $Y$. 
Definição 1.1.3. Uma variedade algébrica afim é um subconjunto irredutível de $\mathbb{C}^{r}$ munido da topologia induzida.

Analogamente ao caso de polinômios, espaços analíticos complexos são definidos pelos zeros de funções analíticas complexas. Neste contexto, precisamos da seguinte definição:

Definição 1.1.4. Consideremos o conjunto dos pares $\left(V_{\alpha}, U_{\alpha}\right)$, onde $U_{\alpha}$ é uma vizinhança aberta da origem em $\mathbb{C}^{r}$ e os $V_{\alpha}$ são subconjuntos de $U_{\alpha}$. Dizemos que dois pares $\left(V_{1}, U_{1}\right) e$ $\left(V_{2}, U_{2}\right)$ são equivalentes se existir uma vizinhança da origem $W \subset U_{1} \cap U_{2}$ tal que $V_{1} \cap W=$ $V_{2} \cap W$. Uma classe de equivalência desta relação é chamada germe na origem em $\mathbb{C}^{r}$.

Definição 1.1.5. Um germe de espaço analítico $(V, 0)$ em torno da origem é o germe do subconjunto

$$
V=\left\{\left(x_{1}, \ldots, x_{r}\right) \in \mathbb{C}^{r} \mid f_{i}\left(x_{1}, \ldots, x_{r}\right)=0,1 \leq i \leq n\right\}
$$

onde $f_{i} \in \mathcal{O}_{r}$ para todo $i$.

Dizemos que um germe de espaço analítico $V$ é irredutivel se para quaisquer $V_{1}$ e $V_{2}$ tais que $V=V_{1} \cup V_{2}$, então $V=V_{1}$ ou $V=V_{2}$. Neste caso, dizemos que $V$ é uma variedade analítica.

Chamamos de germe de espaço analítico em $x$, um germe de conjunto $V$ em $x$ tal que para alguma vizinhança $U$ de $x$ o germe de $V \cap U$ pode ser escrito como o conjunto de zeros de $f_{i} \in \mathcal{O}_{r}, \operatorname{com} 1 \leq i \leq n$.

Dizemos que um ponto $z$ de um espaço analítico $V$ é regular ou suave, se para alguma vizinhança $U$ de $z$, o germe de $U \cap V$ pode ser descrito como o conjunto de zeros de um número finito de germes de funções analíticas que possuem $z$ como ponto regular. Um ponto de $V$ que não é regular é chamado ponto singular de $V$.

Na seqüência, apresentamos o conceito de estratificação de Whitney introduzido por H. Whitney em [53]. A idéia básica na teoria de estratificação é decompor uma variedade singular em variedades regulares, chamadas estratos. Para maiores detalhes sobre estratificações, ver [23].

Definição 1.1.6. Sejam $M$ uma variedade suave e $V \subset M$. Uma estratificação localmente finita de $V$ é uma partição de $V$ em subvariedades de $M$, chamadas estratos, tais que, para todo ponto de $V$ existe uma vizinhança de $M$ que encontra apenas um número finito de estratos.

Definição 1.1.7. Seja $M$ uma variedade suave, $V \subset M e\left\{V_{\alpha}\right\}$ estratificação de $V$. Dizemos que $\left\{V_{\alpha}\right\}$ satisfaz a condição de fronteira se para dois estratos $V_{\alpha}$ e $V_{\beta}$, tais que $V_{\alpha} \cap \bar{V}_{\beta} \neq \emptyset$, então $V_{\alpha} \subset \bar{V}_{\beta}$. 
Definição 1.1.8. Uma estratificação $\left\{V_{\alpha}\right\}$ satisfaz às condições de Whitney se para todo par $\left(V_{\alpha}, V_{\beta}\right)$ de estratos, tais que $V_{\beta}$ esteja no fecho de $V_{\alpha}$ e para todo $y \in V_{\beta}$ temos:

a) Para toda seqüência de pontos $x_{i}$ de $V_{\alpha}$ convergindo para $y$, tal que o limite

$$
\lim _{i \rightarrow \infty} T_{x_{i}}\left(V_{\alpha}\right)=T
$$

existe na Grassmanniana correspondente, então $T$ contém $T_{y}\left(V_{\beta}\right)$.

b) Se além disso, para toda seqüência $y_{i}$ de pontos de $V_{\beta}$ com limite y e tal que a seqüência de direções

$$
\lim _{i \rightarrow \infty} x_{i} y_{i}=\lambda
$$

existe no espaço projetivo, então $T$ contém $\lambda$.

Uma estratificação que satisfaz às condições de Whitney e à condição de fronteira é chamada de estratificação de Whitney ou estratificação Whitney regular.

Em [53], H. Whitney mostrou que toda variedade analítica complexa admite uma estratificação de Whitney.

Podemos agora introduzir o conceito de função analítica complexa com singularidade isolada, definida num germe de espaço analítico complexo $V$ munido de uma estratificação $\left\{V_{\alpha}\right\}$.

Definição 1.1.9. Seja $f: V \longrightarrow \mathbb{C}$ uma função analítica complexa, definida sobre um conjunto analítico estratificado $V$ com estratificação $\left\{V_{\alpha}\right\}$. Dizemos que $f$ tem singularidade isolada em $x \in V$, com respeito à estratificação $\left\{V_{\alpha}\right\}$ se existe $\epsilon>0$ tal que $\left.f\right|_{B_{\epsilon}(x) \cap\left(V_{\alpha} \backslash\{x\}\right)} \longrightarrow \mathbb{C} e ́$ submersão para todo $V_{\alpha} \in\left\{V_{\alpha}\right\}$.

Usando o conceito de função analítica complexa com singularidade isolada com respeito à estratificação $\left\{V_{\alpha}\right\}$, Lê D. T. mostra um teorema que estende o Teorema de Fibração de Milnor para funções definidas em variedades singulares.

Teorema 1.1.1. ([49], pág. 84) Sejam $V$ uma variedade analítica complexa e $f: V \longrightarrow \mathbb{C}$ uma função analítica complexa com singularidade isolada em $x$ relativa à estratificação de Whitney $\left\{V_{\alpha}\right\}$ de $V$. Então, existem números reais $\epsilon$ e $\eta$, suficientemente pequenos, tais que a aplicação

$$
\phi: B_{\epsilon}(x) \cap V \cap f^{-1}\left(D_{\eta}^{*}\right) \longrightarrow D_{\eta}^{*}
$$

induzida por $f$ é uma fibração localmente trivial sobre o disco menos um ponto

$$
D_{\eta}^{*}=\{y \in \mathbb{C}|0<| y \mid<1\} .
$$




\section{$1.2 \quad$ Teoria de Morse}

A Teoria clássica de Morse estabelece conexões entre a topologia de uma variedade suave $M$ e os pontos críticos de funções reais próprias definidas em $M$, chamadas funções de Morse. Em particular, em [36], J. Milnor mostra que informações próximas aos pontos críticos de uma função de Morse $f: M \longrightarrow \mathbb{R}$ podem ser usadas para construir $M$ através de uma seqüência de colagens. Este fato evidencia uma relação entre os pontos críticos de uma função de Morse definida em $M$ e a sua homologia.

Definição 1.2.1. Seja $f: M \longrightarrow N$ uma aplicação diferenciável entre duas variedades.

a) Um ponto $p \in M$ é chamado ponto crítico de $f$ se a derivada $d f(p): T_{p} M \longrightarrow T_{f(p)} N$ não é sobrejetiva. Um ponto que não é crítico é chamado regular.

b) Um ponto $c \in N$ é chamado valor crítico se $f^{-1}(c)$ contém um ponto crítico. Caso contrário c é chamado valor regular. Um valor crítico que tem somente uma imagem inversa é chamado valor crítico simples.

Definição 1.2.2. Seja $f: M \longrightarrow \mathbb{R}$ uma função diferenciável. Um ponto crítico $p \in M$ é chamado não degenerado, se a derivada segunda de $f$ é uma forma bilinear não degenerada.

Uma função cujos pontos críticos são não- degenerados é chamada função de Morse. Seja $p \in M$ um ponto crítico não- degenerado. O número de auto- valores negativos da matriz Hessiana de $f$ em $p$, em algum sistema de coordenadas locais, é chamado de índice do ponto crítico de $f$ em $p$.

Vamos enunciar dois teoremas clássicos da Teoria Clássica de Morse. Seja $f: M \longrightarrow \mathbb{R}$ uma função real suave definida em uma variedade $M$. Denotamos por

$$
M^{a}=f^{-1}(-\infty, a]=\{p \in M: f(p) \leq a\}
$$

Teorema 1.2.1. ([37], pág. 12) Sejam $f: M \longrightarrow \mathbb{R}$ uma função real suave definida em $M$ como acima e $a, b \in \mathbb{R}$ tais que $f^{-1}[a, b]$ é compacto e não contém pontos críticos de $f$. Então, $M^{a}$ é difeomorfo à $M^{b}$. Além disso, $M^{a}$ é retrato por deformação de $M^{b}$.

Quando o intervalo $[a, b]$ contém um valor crítico $f(p)$, o tipo topológico de $M^{b}$ pode ser descrito pelo seguinte teorema.

Teorema 1.2.2. Sejam $f: M \longrightarrow \mathbb{R}$ uma função real suave definida em $M, p$ um ponto crítico não degenerado de índice $\lambda$ e $f(p)=c$. Suponhamos que $f^{-1}[c-\epsilon, c+\epsilon]$ seja compacto 
e não contenha outros pontos críticos de $f$ além de $p$, para algum $\epsilon>0$. Então, para todo $\epsilon$ suficientemente pequeno o conjunto $M^{c+\epsilon}$ tem o mesmo tipo de homotopia do conjunto obtido de $M^{c-\epsilon}$ através da colagem de uma célula de dimensão $\lambda$.

Sejam $M \subset \mathbb{C}^{r}$ uma variedade analítica complexa e $f: M \longrightarrow \mathbb{C}$ uma função analítica definida em $M$. Como conseqüência das equações de Cauchy- Riemann segue que $p$ é ponto crítico de $f$ se, e somente se, $p$ é ponto crítico da parte real de $f$. Assim, podemos aplicar os teoremas acima para variedades analíticas complexas usando a parte real de $f$.

Definição 1.2.3. Um germe de função analítica $f:\left(\mathbb{C}^{r}, 0\right) \longrightarrow(\mathbb{C}, 0)$ é chamado de Morse em relação à $\left\{V_{\alpha}\right\}$, se as seguintes condições são válidas:

a) Se $V_{0} \neq\{0\}$ é o estrato contendo 0 , então a restrição de $f$ ao estrato $V_{0}$ deve ter ponto crítico não degenerado em 0 ;

b) Suponhamos que $\left\{x_{n}\right\}$ seja uma seqüência de pontos em $V_{\alpha}$ convergindo para 0 e que $T$ seja o limite de hiperplanos tangentes $\lim _{n \longrightarrow \infty} T_{x_{n}} V_{\alpha}$. Então, se $0 \notin V_{\alpha}$ a derivada $d f_{0}$ deve ter posto 1 em $T$.

Proposição 1.2.1. ([4], pág. 68) Seja $(X, 0) \subset\left(\mathbb{C}^{r}, 0\right)$ variedade analítica complexa munida de uma estratificação de Whitney $\left\{V_{\alpha}\right\}$ e $f:\left(\mathbb{C}^{r}, 0\right) \longrightarrow(\mathbb{C}, 0)$ uma função analítica tal que dentro de uma bola $B$, contendo a origem, a restrição de $f$ a qualquer estrato tem singularidade somente em 0 . Então, existe uma familia real de funções analíticas $f_{t}:\left(\mathbb{C}^{r}, 0\right) \longrightarrow(\mathbb{C}, 0)$, com $t \in[0, \epsilon)$, tal que $f_{0}=f$ e para $t \neq 0$ a função $f_{t}$ tem somente singularidades de Morse em $X$ dentro de $B$.

\subsection{Multiplicidade Polar}

A noção de variedade polar foi usada por B. Tessier e Lê D. T. nos anos 70, ([48], [47])com a finalidade de estudar singularidades de variedades analíticas. Nesta seção apresentaremos a definição de variedades polares dada por B. Tessier em [47].

Seja $f: X \longrightarrow S$ um morfismo de espaços analíticos reduzidos complexos tais que as fibras de $f$ são suaves e de dimensão $d=\operatorname{dim} X-\operatorname{dim} S$, fora de um conjunto fechado, não denso $F \subset X$. De maneira geral, podemos mergulhar $X \subset S \times \mathbb{C}^{r}$ como no diagrama

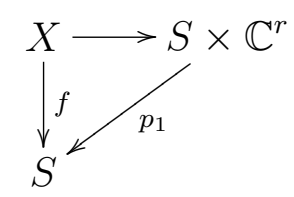


Definição 1.3.1. Seja $D_{d-k+1}$ um subespaço de $\mathbb{C}^{r}$ de codimensão $d-k+1$, com $0 \leq k \leq d$. Definimos

$$
C\left(D_{d-k+1}\right)=\left\{T \in G r\left(d, \mathbb{C}^{r}\right) \mid \operatorname{dim}\left(T \cap D_{d-k+1}\right) \geq k\right\}
$$

onde $\operatorname{Gr}\left(d, \mathbb{C}^{r}\right)$ é a grassmaniana de $d$-planos em $\mathbb{C}^{r}$.

Observemos que se $T$ é um plano de dimensão $d$, a dimensão esperada para $T \cap D_{d-k+1}$ é $d-(d-k+1)=k-1$. Logo, os pontos que estão em $C\left(D_{d-k+1}\right)$ são os pontos que têm contato excepcional com $D_{d-k+1}$.

Sejam $p: \mathbb{C}^{r} \longrightarrow \mathbb{C}^{d-k+1}$ uma projeção linear genérica cujo núcleo é $D_{d-k+1}$. De forma simplificada, uma projeção linear genérica é a projeção menos singular possível (ver [47], pág. 419). Para $s \in X-F$ a fibra $X_{s}$ de $f$ em $X$ é não singular contida em $\{f(s)\} \times \mathbb{C}^{r}$. Denotamos por $p_{s}: X_{s} \longrightarrow \mathbb{C}^{d-k+1}$ a restrição à $X_{s}$ da projeção $p$. Definimos

$$
P_{k}(f, p)^{0}=\left\{x \in X-F \mid x \in \Sigma\left(p_{s}\right)\right\}
$$

e denotamos por $P_{k}(f, p)$ seu fecho em $X$.

Proposição 1.3.1. ([47]], IV 1.3.2) Seja $f:(X, 0) \longrightarrow(S, 0)$ uma aplicação plana com fibras suaves em todo ponto de $X-F$. Então, $P_{k}(f, p)$ é um subconjunto analítico fechado, vazio ou de codimensão pura $k$ em $X$.

Podemos formular a seguinte definição:

Definição 1.3.2. Sejam $f:(X, 0) \longrightarrow(S, 0)$ um morfismo como acima, um $S$ - mergulho $(X, 0) \subset(S, 0) \times\left(\mathbb{C}^{r}, 0\right)$ e um subespaço linear $D_{d-k+1} \subset \mathbb{C}^{r}$ genérico. O subespaço analítico fechado $P_{k}(f, p)$ de $X$ é chamado a variedade polar relativa de $X$ com codimensão $k$ associada a $f$ e $D_{d-k+1}$. Estamos considerando aqui, $D_{d-k+1}$ o núcleo de $p$.

Quando $S$ é um ponto, denominamos $P_{k}(f, p)$ de variedade polar absoluta. Em geral, vamos omitir o adjetivo "relativo ou absoluto".

O invariante chave de $\left.P_{k}(f, p)\right)$ é sua multiplicidade, $m_{0}\left(P_{k}(f, p)\right)$, que é chamada a $k$-ésima multiplicidade polar relativa de $X$ e denotada por $m_{k}(X, f)$. Se $f$ é a aplicação constante, denotamos a multiplicidade por $m_{k}(X)$. Para uma projeção genérica a multiplicidade é independente de $D_{d-k+1}$ e, de fato, é um invariante analítico de $X$.

Seja $X \subset \mathbb{C}^{r}$ uma variedade analítica complexa de dimensão $d$. A variedade polar absoluta de $X$ de codimensão $d$ consiste de um número finito de pontos ou é vazia. Em ambos os casos, sua multiplicidade não está bem definida. Entretanto, T. Gaffney em [19] introduz a $d$ - ésima multiplicidade polar através da seguinte construção: 
Sejam $\mathfrak{X} \subset \mathbb{C}^{r} \times \mathbb{C}^{s}$ uma variedade analítica complexa de dimensão $d+s$ e $f: \mathfrak{X} \longrightarrow \mathbb{C}^{s}$ uma função analítica tal que $f^{-1}(0)=X$. Então, $m_{d}(X, p)=m_{0}\left(P_{d}(f, p)\right)$, onde $P_{d}(f, p)$ é a variedade polar de $\mathfrak{X}$ com relação à $(f, p)$. Se $p$ é genérica, denotamos $m_{d}(X, p)$ simplesmente por $m_{d}(X)$.

\subsection{Anéis Cohen-Macaulay}

Anéis Cohen-Macaulay fornecem um contexto que possibilita a generalização de muitos resultados sobre anéis regulares. Nesta seção revisaremos alguns conceitos básicos sobre estes anéis e maiores detalhes podem ser encontrados em [8].

Dado $R$ um anel comutativo Noetheriano, a definição de Cohen- Macaulay envolve duas constantes: a altura e a profundidade de um ideal arbitrário $I$ de $R$. A noção de altura é topológica, enquanto que a de profundidade está relacionada com o comprimento de uma seqüência regular em $I$. Em 1916, Macaulay provou que álgebras polinomiais sobre um corpo satisfazem propriedades que posteriormente serão chamadas Cohen-Macaulay. Posteriormente, em 1945, Cohen generalizou este resultado e provou que anéis regulares também satisfazem estas mesmas propriedades.

Os anéis Cohen-Macaulay possuem uma rica estrutura e incluem uma grande classe de anéis que aparecem naturalmente em Geometria Algébrica, tais como anéis regulares e intersecções completas.

Definição 1.4.1. A dimensão de Krull (ou simplesmente dimensão) de um anel $R$ é o supremo dos comprimentos das cadeias

$$
P_{r} \supsetneq P_{r-1} \supsetneq \ldots \supsetneq P_{0},
$$

onde cada $P_{i}$ é ideal primo $P_{i} \varsubsetneqq R$ para todo $i$.

Se $I$ é um ideal de $R$, definimos a dimensão de $I$ como sendo a dimensão do anel $R / I$.

Definição 1.4.2. Seja $R$ um anel e $P$ um ideal primo de $R$. Definimos a altura de $P$ pelo supremo das cadeias de ideais

$$
P_{0} \varsubsetneqq P_{1} \cdots \varsubsetneqq P_{m}
$$

tais que $P_{i}$ é ideal primo, para todo $i$ e $P_{m}=P$.

Se $I$ é um ideal arbitrário de $R$, definimos a altura $^{1}$ de $I$ como

$$
h t(I)=\inf \{h t(\mathcal{P}) \mid \mathcal{P} \text { é ideal primo de } R \text { e } \mathcal{P} \supset I\} .
$$

\footnotetext{
${ }^{1}$ Muitos autores usam o termo codimensão de $I$ para $h t(I)$.
} 
Do Teorema do ideal principal generalizado segue que se $I$ pode ser gerado por $n$ elementos, então $h t(I) \leq n$ (ver [8], pág. 412).

Definição 1.4.3. Sejam $R$ um anel e $M$ um $R$-módulo. Uma seqüência $\left(a_{1}, \cdots, a_{n}\right)$ de elementos de $R$ é regular em relação à $M$ ou uma $M$ - seqüência se

1) $a_{i}$ não é um divisor de zero em $M /\left\langle a_{1}, \cdots, a_{i-1}\right\rangle M$ para $i=1, \cdots, n$;

2) $M \neq\left\langle a_{1}, \cdots, a_{n}\right\rangle M$.

Se $R$ é um anel Noetheriano e $\mathbf{x}=\left(x_{1}, \ldots, x_{n}\right)$ é uma $M$-seqüência, então podemos estender a seqüência ascendente $\left(x_{1}\right) \subset\left(x_{1}, x_{2}\right) \subset \ldots \subset\left(x_{1}, \ldots, x_{n}\right)$ para uma $M$-seqüência maximal.

Teorema 1.4.1. ([8], pág. 10)(Rees) Seja $R$ um anel Noetheriano, $M$ um R-módulo finito e I um ideal tal que $I M \neq M$. Então, todas as $M$-seqüências em I têm o mesmo comprimento.

Usando o resultado do teorema anterior, podemos definir:

Definição 1.4.4. Sejam $R$ um anel Noetheriano, $M$ um $R$-módulo finito e I um ideal tal que $I M \neq M$. Então o comprimento comum de todas as $M$-seqüências maximais em I é chamado profundidade de $I$, denotado por depth $(I, M)$.

Quando $M=R$, escrevemos simplesmente $\operatorname{depth}(I)$. Se $M=I M$, então $\operatorname{depth}(I, M)$ é, por convenção, infinito. Quando $(R, \mathfrak{M})$ é um anel local, então o depth(M $M$, é simplesmente denotado por depth $(M)$.

Definição 1.4.5. Uma resolução projetiva de um $R$-módulo $M$ é um complexo

$$
\mathcal{F}: \ldots \longrightarrow F_{n} \stackrel{\phi_{n}}{\longrightarrow} F_{n-1} \stackrel{\phi_{n-1}}{\longrightarrow} \ldots \longrightarrow F_{1} \stackrel{\phi_{1}}{\longrightarrow} F_{0} \longrightarrow M \longrightarrow 0
$$

de $R$-módulos projetivos tal que coker $\phi_{1}=M$ e $\mathcal{F}$ tem homologia nula. ${ }^{2}$ Se cada módulo $F_{i}$ é livre, então $\mathcal{F}$ é chamada uma resolução projetiva livre.

Dizemos que $\mathcal{F}$ é uma resolução projetiva finita de comprimento $n$, se $F_{n+1}=0$ e $F_{i} \neq 0$ para $0 \leq i \leq n$. A dimensão projetiva de $M$, denotada por $p d(M)$, é o mínimo dos comprimentos das resoluções projetivas de $M$. Se $M$ não tem resolução projetiva finita, então definimos $p d(M)=\infty$.

Definição 1.4.6. Seja $R$ um anel Noetheriano local e $M$ um $R$-módulo não nulo. Dizemos que $M$ é perfeito se $p d(M)=\operatorname{depth}(M)$. Se I é um ideal de $R$, então I é perfeito se depth $(I)=$ $\operatorname{pd}(R / I)$.

\footnotetext{
${ }^{2} \mathrm{~A}$ homologia do complexo em $F_{n}$ é o módulo $\operatorname{ker}\left(\phi_{n}\right) / \operatorname{Im}\left(\phi_{n+1}\right)$.
} 
O seguinte teorema é a fórmula de Auslander-Buchsbaum. Ela é uma ferramenta importante no estudo de módulos de dimensão projetiva finita e na demonstração do Teorema de HilbertBurch que é fundamental no nosso estudo.

Teorema 1.4.2. (Fórmula de Auslander-Buschsbaum) ([15], pág 479) Seja (R, M) um anel local. Se $M$ é um R-módulo finitamente gerado de dimensão projetiva finita, então

$$
\operatorname{pd}(M)=\operatorname{depth}(\mathfrak{M}, R)-\operatorname{depth}(\mathfrak{M}, M) .
$$

Proposição 1.4.1. ([15], pág. 453) Seja $R$ um anel e $M$ um $R$-módulo finitamente gerado. Se $I$ é um ideal de $R$ contendo ann $(M)$, então depth $(I, M)$ é menor ou igual ao comprimento de qualquer cadeia descendente de ideais em $P$, onde $P$ é ideal primo contendo I e P é primo associado de $M$. Em particular, depth $(I, R) \leq h t(I)$.

Definição 1.4.7. Dizemos que um anel Noetheriano $R$ é um anel Cohen-Macaulay se depth $(\mathcal{M})=$ $\operatorname{codim}(\mathcal{M})$ para todo ideal maximal $\mathcal{M}$ de $R$.

Teorema 1.4.3. ([8], pág 59) Sejam $R$ um anel Cohen-Macaulay e $M$ um R-módulo finito de dimensão projetiva finita.

a) Se $M$ é perfeito, então ele é Cohen-Macaulay.

b) A recíproca é verdadeira quando $R$ é local.

Dizemos que um anel $R$ é uma intersecção completa se existe um anel regular $S$ e uma seqüência regular $x_{1}, \cdots, x_{n} \in S$ tais que $R \cong S /\left(x_{1}, \cdots, x_{n}\right)$. O anel $R$ é localmente uma intersecção completa se para cada ideal maximal $\mathcal{M} \subset R$ a localização de $R$ em $\mathcal{M}$ é uma intersecção completa. É possível mostrar que, localmente, uma intersecção completa é CohenMacaulay.

Definição 1.4.8. Uma variedade complexa analítica $X$ é chamada normal no ponto $x \in X$ se o anel local de $X$ em $x$ é integralmente fechado. Dizemos que $X$ é normal se ela é normal em todo ponto.

Notemos que se $X$ é não singular em $x$, então $X$ é normal em $x$, uma vez que anéis regulares são integralmente fechados. Em 1951, K. Oka provou que se $X$ é uma hipersuperfície complexa cujo conjunto singular tem codimensão maior ou igual a dois, então $X$ é uma variedade analítica 
normal. Posteriormente, este resultado foi generalizado por S. Abhyankar e W. Thimm para o caso de intersecções completas. Quando $X$ é uma variedade Cohen-Macaulay, temos o seguinte resultado.

Teorema 1.4.4. ([35], pág. 487) Sejam $X$ um espaço analítico complexo reduzido e $x \in X$. Suponhamos que $\mathcal{O}_{X, x}$ é um anel Cohen-Macaulay e que a codimensão do conjunto singular de $X$ em $x$ é maior ou igual a dois. Então, $X$ é normal em $x$.

\subsection{Ideais Determinantais}

Nesta seção, consideramos $M$ uma matriz com entradas no anel de indeterminadas sobre um corpo e definimos as variedades determinantais. Estudamos alguns resultados clássicos obtidos por J. Eagon e Hochster que mostram que tais variedades são Cohen- Macaulay. Em particular, no caso em que $X$ é Cohen-Macaulay, de codimensão 2, o Teorema de Hilbert- Burch fornece uma descrição eficiente deste tipo de singularidades.

Seja $\operatorname{Mat}_{(n, p)}(\mathbb{C})$ o conjuntos de todas as matrizes $n \times p$ com entradas complexas. Indiquemos por $\Delta_{t}$ o subconjunto de $\operatorname{Mat}_{(n, p)}(\mathbb{C})$ formado pelas matrizes que têm posto menor que $t$, com $1 \leq t \leq \min \{n, p\}$. O conjunto $\Delta_{t}$ é denominado variedade determinantal genérica.

Definição 1.5.1. Sejam $M=\left(m_{i j}(x)\right)$ uma matriz $n \times p$ cujas entradas são funções analíticas complexas em $U \subset \mathbb{C}^{r}$ e $f$ a função cujas coordenadas são os menores $t \times t$ da matriz $M$. Dizemos que $X$ é uma variedade determinantal de codimensão $(n-t+1)(p-t+1)$ se $X$ é definida pelas equações $f=0$.

Podemos olhar a matriz $M$ como uma aplicação $M: \mathbb{C}^{r} \longrightarrow \operatorname{Mat}_{(n, p)}(\mathbb{C}), \operatorname{com} M(0)=0$. Então, a variedade determinantal é o conjunto $X=M^{-1}\left(\Delta_{t}\right)$, com $1 \leq t \leq \min (n, p)$. O conjunto singular de $X$ é dado por $M^{-1}\left(\Delta_{t-1}\right)$. Denotamos $X_{r e g}=M^{-1}\left(\Delta_{t} \backslash \Delta_{t-1}\right)$ a parte regular de $X$. Notemos que se $X$ tem singularidade isolada na origem, então $r \leq(n-t+2)(p-$ $t+2)$.

Proposição 1.5.1. Se $1 \leq t \leq \min \{n, p\}-1$, então o conjunto $\Delta_{t+1}$ é uma subvariedade algébrica irredutivel de $\operatorname{Mat}_{(n, p)}(\mathbb{C})$ de codimensão $(n-t)(p-t)$.

\section{Demonstração.}

Sejam $\operatorname{Gr}\left(d, \mathbb{C}^{r}\right)$ a variedade grassmanniana dos $d$ - planos em $\mathbb{C}^{r}$,

$$
\widetilde{\Delta}_{t+1}=\left\{(A, W) \in \operatorname{Mat}_{(n, p)}(\mathbb{C}) \times G r\left(p-t, \mathbb{C}^{p}\right): A(W)=0\right\}
$$


e $\pi_{2}: \widetilde{\Delta}_{t+1} \longrightarrow G r(p-t, p)$ a projeção no segundo fator.

A projeção $\pi_{2}$ define em $\widetilde{\Delta}_{t+1}$ a estrutura de fibrado vetorial sobre $\operatorname{Gr}\left(p-t, \mathbb{C}^{p}\right)$ de posto $p t$. Logo, a variedade $\widetilde{\Delta}_{t+1}$ é suave, conexa e tem dimensão $t(n+p-t)$.

Por outro lado, se

$$
\pi_{1}: \operatorname{Mat}_{(n, p)}(\mathbb{C}) \times \operatorname{Gr}(p-t, p) \longrightarrow \operatorname{Mat}_{(n, p)}(\mathbb{C}),
$$

é a projeção no primeiro fator, então $\pi_{1}$ envia $\widetilde{\Delta}_{t+1}$ propriamente em $\Delta_{t}$. Portanto, $\Delta_{t}$ é uma variedade algébrica irredutível de $\operatorname{Mat}_{(n, p)}(\mathbb{C})$. Além disso, se $A \in \Delta_{t+1} \backslash \Delta_{t}$, então existe apenas um par na imagem inversa $\pi_{1}^{-1}(A) \in \Delta_{t+1}$, a saber, $(A, \operatorname{ker}(A))$.

Nosso próximo objetivo é determinar o espaço tangente de $\Delta_{t+1}$ nos seus pontos regulares. A demonstração segue da identificação entre o espaço tangente a $G r\left(p-t, \mathbb{C}^{p}\right)$ em $W$ e $\operatorname{Hom}\left(W, \mathbb{C}^{r} / W\right)$ e do cálculo do espaço tangente à $\widetilde{\Delta}_{t}$ em cada ponto.

Proposição 1.5.2. ([1], pág. 68) O conjunto singular de $\Delta_{t}$ é exatamente $\Delta_{t-1}$ e para $A \in$ $\Delta_{t} \backslash \Delta_{t-1}$, então

$$
T_{A} \Delta_{t}=\left\{B \in \operatorname{Mat}_{(n, p)}(\mathbb{C}) \mid B(k e r(A)) \subset A\left(\mathbb{C}^{r}\right)\right\}
$$

O seguinte resultado foi obtido por J. Eagon em [12]:

Teorema 1.5.1. ([7], pág. 13) Sejam $R$ um anel Noetheriano e $M$ uma matriz $n \times p$ com entradas em $R$. O ideal I, gerado pelos menores maximais de R, é perfeito.

No caso em que as entradas de $M$ são indeterminadas sobre um corpo e $I$ é o ideal gerado pelos menores maximais de $M$ é chamado ideal determinantal. Em [11], J. Eagon e D. G. Northcott constroem uma resolução para $I$ e provam a perfeição destes ideais. Neste caso, esta condição é equivalente a dizer que o anel local da variedade determinada por $I$ é CohenMacaulay. Posteriormente, em [27], J. Eagon e Hoschester generalizam este resultado para variedades definidas por menores $t \times t$ de matrizes $n \times p$ onde $t \leq \min \{n, p\}$.

Dentro da classe de variedades determinantais, estamos particularmente interessados em estudar aquelas que são Cohen- Macaulay de codimensão 2. O teorema de Hilbert-Burch fornece uma descrição conveniente desses germes e de suas perturbações.

Teorema 1.5.2. (Hilbert-Burch) ([15], pág. 506)

a. Se um complexo

$$
\mathcal{F}: 0 \longrightarrow F_{2} \stackrel{\varphi_{2}}{\longrightarrow} F_{1} \stackrel{\varphi_{1}}{\longrightarrow} R \longrightarrow R / I \longrightarrow 0 ;
$$


é exato e $F_{1} \cong R^{n}$, então $F_{2} \cong R^{n-1}$ e existe $\boldsymbol{a} \in I$ não divisor de zero tal que $I=$ $\boldsymbol{a} I_{n-1}\left(\varphi_{2}\right)$, onde $I_{n-1}\left(\varphi_{2}\right)$ é o ideal gerado pelos menores de tamanho $n-1$ da matriz de $\varphi_{2}$. De fato, a i-ésima entrada da matriz de $\varphi_{1}$ é $(-1)^{i} a$ vezes o menor obtido de $\varphi_{2}$ retirando-se a $i$-ésima linha. O ideal $I_{n-1}\left(\varphi_{2}\right)$ tem profundidade exatamente 2 , isto é, $\operatorname{depth}(I)=2$.

b. Reciprocamente, dada $\varphi_{2}$ uma matriz $(n-1) \times n$ tal que depth $I_{n-1}\left(\varphi_{2}\right) \geq 2$ e um elemento a que não é divisor de zero, a aplicação $\varphi_{1}$ obtida como na parte (a) torna $\mathcal{F}$ uma resolução livre de $R / I$, com $I=\boldsymbol{a} I_{n-1}\left(\varphi_{2}\right)$.

O teorema anterior é muitas vezes utilizado da seguinte forma: Seja um anel regular $S$, e $R=S / I$ um anel fatorial Cohen-Macaulay (usualmente tomado como sendo local ou graduado) tal que $\operatorname{cod}(I)=2$. Então, usando a Fórmula de Auslander- Buchsbaum temos

$$
\operatorname{pd}(R)=\operatorname{depth}(P, S)-\operatorname{depth}(P, R)=2
$$

e o teorema de Hilbert-Burch se aplica.

Reciprocamente, dada uma matriz $(n-1) \times n$ cujos menores $(n-1) \times(n-1)$ geram um ideal $I$ de codimensão 2 num anel regular $S$, então $S / I$ será Cohen-Macaulay de codimensão 2. (Esta recíproca pode ser generalizada para matrizes de outras dimensões e outros tipos de ideais associados).

Observação 1.5.1. Seja $M$ uma matriz $n \times p$ e $I$ um ideal de $\mathcal{P}$ gerado pelos menores $t \times t$ de $I$, com $t \leq \min \{n, p\}$. Chamamos de operações elementares entre colunas as seguintes operações:

i) Permutações entre colunas;

ii) A substituição de $C_{j}$ por $u C_{j}+v C_{i}$, onde $C_{i}$ e $C_{j}$ denotam colunas distintas de $M$, $1 \leq j, i \leq p, i \neq j$, v é unidades em $\mathcal{P}$ e $u \in \mathcal{P}$.

Operações elementares entre linhas são definidas da mesma forma. Então,

a) O ideal determinantal I é invariante sob operações elementares entre linhas e colunas.

b) Se uma das entradas da matriz $M$ é uma unidade em $\mathcal{P}$, podemos transformar $M$ por um número finito de operações entre linhas e colunas para a seguinte forma:

$$
M^{\prime}=\left(\begin{array}{c|ccc}
1 & 0 & \ldots & 0 \\
\hline 0 & & & \\
\vdots & & \widetilde{M} & \\
0 & & &
\end{array}\right)
$$


e, I é igual ao ideal gerado pelos menores $(t-1) \times(t-1)$ de $M^{\prime}$. Portanto, sem perda de generalidade, na maior parte do trabalho vamos considerar matrizes cujas entradas pertencem ao ideal maximal de $\mathcal{P}$.

c) Notemos que é equivalente considerar matrizes $(n+1) \times n$ ou $n \times(n+1)$. Na maior parte deste trabalho usamos a notação $n \times(n+1)$. 


\section{Capítulo 2}

\section{Teoria de Singularidades de Matrizes}

\subsection{Notações}

Os resultados deste capítulo, em sua maioria, se verificam para singularidades reais e complexas. Denotamos $G L_{i}(\mathcal{P})$, o grupo de matrizes invertíveis $i \times i$ com entradas em $\mathcal{P}=\mathcal{O}_{r}$ ou $\mathcal{E}_{r}$.

Seja $\mathcal{R}$ o grupo de mudanças de coordenadas em $\left(\mathbb{K}^{r}, 0\right)$, isto é, $\mathcal{R}$ é o grupo formado pelos germes de difeomorfismos que são analíticos ou $\mathcal{C}^{\infty}$ se $\mathbb{K}=\mathbb{C}$ ou $\mathbb{R}$, respectivamente.

Além disso, sejam $\mathcal{H}=\mathrm{GL}_{p}(\mathcal{P}) \times \mathrm{GL}_{n}(\mathcal{P})$ e denotamos por $\operatorname{Mat}_{(n, p)}(\mathcal{P})$ o conjunto de todas as matrizes $n \times p$ com entradas em $\mathcal{P}$.

Dados dois germes de singularidades de matrizes, estamos interessados em classificar estes germes segundo a equivalência que definiremos a seguir.

Definição 2.1.1. Seja $\mathcal{G}_{(n, p)}=\mathcal{R} \times G L_{p}(\mathcal{P}) \times G L_{n}(\mathcal{P})$. Dizemos que dois germes de singularidades $M_{1}, M_{2} \in \operatorname{Mat}_{(n, p)}(\mathcal{P})$ são $\mathcal{G}_{(n, p)}$-equivalentes se, e somente se, existe $(\phi, R, L) \in \mathcal{G}_{(n, p)}$ tal que $M_{1}=L^{-1}\left(\phi^{*} M_{2}\right) R$.

$\operatorname{Dados}(\psi, S, N), \quad(\phi, R, L) \in \mathcal{G}_{(n, p)}$ podemos definir a composição,

$$
(\psi, S, N) \circ(\phi, R, L)=\left(\phi \circ \psi,\left(\psi^{*} R\right) S,\left(\psi^{*} L\right) N\right)
$$

Com esta operação, o conjunto $\mathcal{G}_{(n, p)}$ tem estrutura de grupo. Logo, a aplicação

$$
\begin{gathered}
\mathcal{G}_{(n, p)} \times \operatorname{Mat}_{(n, p)}(\mathcal{P}) \longrightarrow \operatorname{Mat}_{(n, p)}(\mathcal{P}) \\
((\phi, R, L), M) \longmapsto L^{-1}\left(\phi^{*} M\right) R
\end{gathered}
$$

é uma ação do grupo $\mathcal{G}_{(n, p)}$ sobre o espaço das matrizes $n \times p$ com entradas em $\mathcal{P}$. Assim, dois germes de matrizes são $\mathcal{G}_{(n, p)}$-equivalentes se, e somente se, eles estão em uma mesma órbita 
sob esta ação. Quando não houver perigo de confusão denotaremos por simplicidade $\mathcal{G}_{(n, p)}$ por $\mathcal{G}$. Se $n=p$, esta equivalência coincide com a $\mathcal{G}$-equivalência definida em [3].

\section{$2.2 \mathrm{O} \mathcal{G}$ - Espaço Tangente}

Para resolver o problema de classificação, a primeira tarefa é determinar o espaço tangente sob a ação do grupo $\mathcal{G}_{(n, p)}$. Seja $\mathcal{P}^{n p}$ o conjunto dos germes $f: \mathbb{K}^{r} \longrightarrow \mathbb{K}^{n p}$ que são analíticos ou $C^{\infty}(\mathbb{K}=\mathbb{C}$ ou $\mathbb{R}$, respectivamentente).

Uma matriz $M \in \operatorname{Mat}_{(n, p)}(\mathcal{P})$ pode ser vista como uma aplicação $f: \mathbb{K}^{r} \longrightarrow \mathbb{K}^{n p}$. Podemos identificar $\operatorname{Mat}_{(n, p)}(\mathcal{P})$ com $\mathcal{P}^{n p}$ e o grupo $\mathcal{G}_{(n, p)}$ pode ser visto como um subgrupo do grupo de contato correspondente. Mais precisamente, vale o seguinte:

Lema 2.2.1. Seja $\mathbb{K}$ o corpo dos números reais ou complexos. O grupo $\mathcal{G}_{(n, p)}$ age no espaço das aplicações $\left(\mathbb{K}^{r}, 0\right) \longrightarrow \mathbb{K}^{n p}$ como subgrupo do grupo de contato correspondente.

O espaço tangente será visto como um $\mathcal{P}$-submódulo de $\mathcal{P}^{n p}$. Seja $C_{i j}(A)$ (respectivamente $\left.R_{l k}(A)\right)$ a matriz que tem $i$-ésima coluna (respectivamente $l$-ésima linha) igual a $j$-ésima coluna de $A$ (respectivamente $k$-ésima linha) com zeros em qualquer outro lugar.

Dada uma matriz $M \in \operatorname{Mat}_{(n, p)}(\mathcal{P})$, escrevemos $M_{x(i)}$ para a matriz $\frac{\partial M}{\partial x_{i}}$.

Proposição 2.2.1. $\quad$ 1. O $\mathcal{R}$-espaço tangente à órbita de um elemento de $M \in M_{(n, p)}(\mathcal{P})$ é o $\mathcal{P}$-módulo gerado por $x_{j} M_{x(i)}, 1 \leq i, j \leq r$.

2. O espaço tangente à órbita de $M$ sob a ação do subgrupo $\mathcal{H} \subset \mathcal{G}$ é o $\mathcal{P}$-módulo gerado por $C_{i j}$, e $R_{l k}$.

\section{Demonstração.}

Os vetores no espaço tangente da $\mathcal{R}$ - órbita são obtidos da forma usual (ver, [22]), isto é, $T \mathcal{R} M=\mathfrak{M}\left\{M_{x(i)} \mid 1 \leq i \leq r\right\}$. Para determinar os outros vetores que compõem o espaço tangente, consideremos a ação do grupo $\mathcal{H}=G L_{p}(\mathcal{P}) \times G L_{n}(\mathcal{P})$. Para cada matriz $M \in$ $\operatorname{Mat}_{(n, p)}(\mathcal{P})$ consideremos inicialmente somente a ação à direita, isto é,

$$
\begin{aligned}
h_{M}: G L_{p}(\mathcal{P}) & \longrightarrow \operatorname{Mat}_{(n, p)}(\mathcal{P}) \\
R & \longmapsto M R .
\end{aligned}
$$

Tomemos um caminho $\gamma(t)=I_{n}+t E_{i j}$, onde $I_{n}$ é a matriz identidade $n \times n$ e $E_{i j}$ é a matriz $n \times n$ com 1 na entrada $(i, j)$ e zero em qualquer outro lugar. Temos,

$$
\left(h_{M} \circ \gamma\right)(t)=h_{M}\left(I_{n}+t E_{i j}\right)=M+t M E_{i j},
$$


$\log \mathrm{O}$

$$
\left(h_{M} \circ \gamma\right)^{\prime}(t)=M E_{i j}=C_{j i} \text {, onde } 1 \leq i, j \leq p .
$$

De forma análoga, se considerarmos a ação à esquerda, obteremos as matrizes $R_{l k}$. Portanto, o $\mathcal{G}_{(n, p)}$-espaço tangente é dado por $(2.3)$.

Definição 2.2.1. O $\mathcal{G}$-espaço tangente a órbita de $M$ é definido por:

$$
T \mathcal{G} M=\mathfrak{M}\left\{M_{x(i)} \mid 1 \leq i \leq r\right\}+\mathcal{P}\left\{R_{l k}, C_{i j} \mid 1 \leq i, j \leq p \quad \text { e } 1 \leq l, k \leq n\right\}
$$

Segue da discussão acima que o $\mathcal{G}_{(n, p)}$-espaço tangente estendido a um germe $M$ é dado por

$$
\mathcal{T}_{e} \mathcal{G}_{(n, p)} \cdot M=\mathcal{P}\left\{M_{x_{(i)}}, R_{l k}, C_{i j}\right\}
$$

Podemos escrever a expressão do espaço tangente de uma outra forma. Dada uma matriz $M \in \operatorname{Mat}_{(n, p)}(\mathcal{P})$, seja $h_{M}$ a aplicação definida em 2.1. Consideremos o caminho pela identidade $\gamma(t)=\left(I_{p}+t A, I_{n}+t B\right)$, onde $t$ é pequeno e $A \in, B \in \operatorname{Mat}_{(n, p)}(\mathcal{P})$. Note que, $\gamma(0)=I d$, $\gamma^{\prime}(0)=(A, B)$ e $\left(d h_{M}\right)(A, B)=(\theta \circ \gamma)^{\prime}(0)$.

Mas,

$$
\left(h_{M} \circ \gamma\right)(t)=h_{M}\left(I d_{n}+t A, I d_{p}+t B\right)=M+t B M+t^{2} B M A+t M A,
$$

então

$$
\left(h_{M} \circ \gamma\right)^{\prime}(0)=B M+M A
$$

Dessa forma, o espaço tangente também pode ser dado por

$$
\mathcal{T} \mathcal{G}_{(n, p)} \cdot M=\mathfrak{M} M_{x_{(i)}}+\mathcal{P}\left\{B M+M A: A, B \in \operatorname{Mat}_{(n, p)}(\mathcal{P})\right\}
$$

Definição 2.2.2. Dizemos que um germe de matriz $M$ é $\mathcal{G}$-estável se $\mathcal{T}_{e} \mathcal{G}_{(n, p)} . M=M a t{ }_{(n, p)}(\mathcal{P})$, isto é,

$$
\mathcal{P}\left\{M_{x_{(i)}}, R_{l k}, C_{i j}\right\}=\operatorname{Mat}_{(n, p)}(\mathcal{P})
$$

\subsection{Determinação Finita}

Nosso próximo objetivo é escrever o teorema de determinação finita para família de matrizes. Nesta seção, vamos nos restringir ao caso analítico, isto é, $\mathcal{P}=\mathcal{O}_{r}$. 
Definição 2.3.1. Um germe de matriz $M \in \operatorname{Mat}_{(n+1, n)}(\mathcal{P})$ é $k$-determinado, ou $k$ - $\mathcal{G}$-determinado se para toda matriz $N$ tal que $j^{k} M(0)=j^{k} N(0), N$ é $\mathcal{G}$-equivalente a $M$. Se $M$ é $k-$ determinado para algum $k$, dizemos que $M$ é $\mathcal{G}$ - finitamente determinado.

Em [3] e [5], a caracterização dos germes finitamente determinados é feita seguindo as idéias dadas por Gaffney em [52]. Nossa demonstração é uma adaptação da demonstração dada em $[16]$.

Consideremos $M \in \operatorname{Mat}_{(n, p)}(\mathcal{P})$ e seja $g=g_{M}$ a aplicação definida por

$$
\begin{gathered}
g: \operatorname{Mat}_{(p, p)}(\mathcal{P}) \times \operatorname{Mat}_{(n, n)}(\mathcal{P}) \longrightarrow \operatorname{Mat}_{(n, p)}(\mathcal{P}) \\
(A, B) \longmapsto B M+M A .
\end{gathered}
$$

Notemos que $C_{j l}(M)=M E_{l j}$ e $R_{i j}(M)=E_{i j} M$, onde como antes $E_{i j}$ é a matriz que tem 1 na entrada $(i, j)$ e zero em qualquer outro lugar.

Para estabelecer os próximos resultados, denotamos por $\mathbb{K}\left\{x_{1}, \ldots, x_{r}\right\}$ o anel das séries de potências convergentes em $r$ variáveis sobre $\mathbb{K}$ e por $\mathbb{K}\left[\left[x_{1}, \ldots, x_{r}\right]\right]$ o anel das séries formais de polinômios em $r$ variáveis.

Lema 2.3.1 (Trivialidade Local). Sejam c $\geq 0$ um inteiro e $M \in \operatorname{Mat}_{(n, p)}\left(\mathbb{K}\left\{x_{1}, \ldots, x_{r}, y_{1}, \ldots, y_{k}\right\}\right)$ definindo uma família com singularidade isolada, onde os $y_{i}$ são vistos como parâmetros. Então as seguintes afirmações são equivalentes:

1. $\frac{\partial M}{\partial y_{j}} \in \mathfrak{M}^{c}\left(\frac{\partial M}{\partial x_{1}}, \ldots, \frac{\partial M}{\partial x_{r}}\right)+\operatorname{Im}(g)$, onde $1 \leq j \leq k$.

2. Existem $\phi_{1}, \ldots, \phi_{r} \in \mathbb{C}\left\{x_{1}, \ldots, x_{r}, y_{1}, \ldots, y_{k}\right\}, A \in \operatorname{Mat}_{(n, n)}\left(\mathbb{K}\left\{x_{1}, \ldots, x_{r}, y_{1}, \ldots, y_{k}\right\}\right)$ e $B \in \operatorname{Mat}_{(p, p)}\left(\mathbb{K}\left\{x_{1}, \ldots, x_{r}, y_{1}, \ldots, y_{k}\right\}\right)$ tais que

- $A\left(x_{1}, \ldots, x_{r}, 0, \ldots, 0\right)=I d_{n}$;

- $B\left(x_{1}, \ldots, x_{r}, 0, \ldots, 0\right)=I d_{p}$;

- $\phi_{i}\left(x_{1}, \ldots, x_{r}, 0, \ldots, 0\right)=x_{i}$;

- $\phi_{i}-x_{i} \in \mathfrak{M}^{c}$ para todo $1 \leq i \leq r$;

- $A M\left(x_{1}, \ldots, x_{r}, y_{1}, \ldots, y_{k}\right) B=M\left(\phi_{1}, \ldots, \phi_{r}, 0, \ldots, 0\right)$.

\section{Demonstração.}

Vamos inicialmente tomar $\mathbb{K}=\mathbb{C}$ e provar o teorema para germes de matrizes com entradas em $\mathbb{C}\left\{x_{1}, \ldots, x_{r}, y_{1}, \ldots, y_{k}\right\}$. É suficiente provar o resultado no anel das séries de potências formais, e utilizar o Teorema de Aproximação de Artin para obter o resultado em $\mathbb{K}\left\{x_{1}, \ldots, x_{r}\right\}$. 
2) $\Longrightarrow 1$ ) Vamos provar esta implicação pelo cálculo direto, porém tomando cuidado de lembrar que estamos trabalhando com matrizes.

Por hipótese, $\phi_{i}\left(x_{1}, \ldots, x_{r}, 0, \ldots, 0\right)=x_{i}$, para qualquer $1 \leq i \leq r$, então $\phi:=\left(\phi_{1}, \ldots, \phi_{r}, y_{1}, \ldots, y_{k}\right)$ é um automorfismo de $\mathbb{K}\left[\left[x_{1}, \ldots, x_{r}, y_{1}, \ldots, y_{k}\right]\right]$.

Denotando o inverso de $\phi$ por $\underline{\psi}=\left(\psi_{1}, \ldots, \psi_{r}, y_{1}, \ldots, y_{k}\right)$, obtemos que $\psi_{i}-x_{i} \in \mathfrak{M}^{c}$, para todo $1 \leq i \leq r \mathrm{e}$

$$
A\left(\psi_{1}, \ldots, \psi_{r}, y_{1}, \ldots, y_{k}\right) M\left(\psi_{1}, \ldots, \psi_{r}, y_{1}, \ldots, y_{k}\right) B\left(\psi_{1}, \ldots, \psi_{r}, y_{1}, \ldots, y_{k}\right)=M\left(x_{1}, \ldots, x_{r}, 0 \ldots, 0\right)
$$

Como o lado direito da última equação independe de $y_{1}, \ldots, y_{k}$ tomando a derivada parcial em relação à $y_{i}$, com $1 \leq i \leq k$ arbitrário, chegamos ao seguinte sistema de $n p$ equações:

$$
\begin{aligned}
& \left(\frac{\partial A}{\partial y_{i}}(\underline{\psi})+\sum_{j=1}^{r} \frac{\partial A}{\partial x_{j}}(\underline{\psi}) \frac{\partial \psi_{j}}{\partial y_{i}}\right) M(\underline{\psi}) B(\underline{\psi})+A(\underline{\psi})\left(\frac{\partial M}{\partial y_{j}}(\underline{\psi})+\sum_{j=1}^{r} \frac{\partial M}{\partial x_{j}}(\underline{\psi}) \frac{\partial \psi_{j}}{\partial i}\right) B(\underline{\psi})+ \\
& +A(\underline{\psi}) M(\underline{\psi})\left(\frac{\partial B}{\partial y_{i}}(\underline{\psi})+\sum_{j=1}^{r} \frac{\partial B}{\partial x_{j}}(\underline{\psi}) \frac{\partial \psi_{j}}{\partial y_{i}}\right)=0
\end{aligned}
$$

onde, por abuso de notação, $\frac{\partial \psi_{j}}{\partial y_{i}}$ denota a multiplicação de todas as entradas da matriz pela série de potências $\frac{\partial \psi_{j}}{\partial y_{i}}$.

Compondo (2.8) com o automorfismo $\phi$ e multiplicando à esquerda por $A^{-1}$ e por $B^{-1}$ à direita, obtemos:

$$
A^{-1}\left(\frac{\partial A}{\partial y_{i}}+\sum_{j=1}^{r} \frac{\partial A}{\partial x_{j}} \frac{\partial \psi_{j}}{\partial y_{i}}(\phi)\right) M+\left(\frac{\partial M}{\partial y_{j}}+\sum_{j=1}^{r} \frac{\partial M}{\partial x_{j}} \frac{\partial \psi_{j}}{\partial_{i}}(\phi)\right)+M\left(\frac{\partial B}{\partial y_{i}}+\sum_{j=1}^{r} \frac{\partial B}{\partial x_{j}} \frac{\partial \psi_{j}}{\partial y_{i}}(\phi)\right) B^{-1}=0
$$

Portanto,

$$
\frac{\partial M}{\partial y_{i}} \in \mathfrak{M}^{c}\left(\frac{\partial M}{\partial x_{1}}, \ldots, \frac{\partial M}{\partial x_{N}}\right)+\operatorname{Im}(g) .
$$

1) $\Longrightarrow$ 2) Provaremos esta implicação usando a construção direta de $\phi, A$ e $B$. Vamos construí-las usando indução sobre o número de parâmetros. Seja,

$$
\begin{aligned}
& A^{(0)}=I d_{n}, \\
& B^{(0)}=i d_{p}, \\
& \phi^{(0)}=\left(x_{1}, \ldots, x_{r}, y_{1}, \ldots, y_{k}\right) .
\end{aligned}
$$


Suponha que $A^{(l)}, B^{(l)}$ e $\phi^{(l)}$ já estejam construídas e tais que

$$
\begin{aligned}
& A^{(l)}(\underline{x}, \underline{0})=I d_{n}, \\
& B^{(l)}(\underline{x}, \underline{0})=I d_{p}, \\
& \phi_{i}^{(l)}(\underline{x}, \underline{0})=x_{i}, \\
& \phi_{i}^{(l)}-x_{i} \in \mathfrak{M}^{c}, \\
& A^{(l)} M B^{(l)}=M\left(\phi_{1}^{l}, \ldots, \phi_{r}^{l}, y_{1}, \ldots, y_{k-l}, 0, \ldots, 0\right) .
\end{aligned}
$$

Por hipótese, sabemos que existem $\xi_{1}, \ldots, \xi_{r} \in \mathfrak{M}^{c} \mathbb{K}[[\underline{x}, \underline{y}]], L \in \operatorname{Mat}_{(n, n)}(\mathbb{K}[[\underline{x}, \underline{y}]])$ e $R \in$ $\operatorname{Mat}_{(p, p)}(\mathbb{K}[[\underline{x}, \underline{y}]])$ tais que

$$
\frac{\partial M}{\partial y_{k-l}}=L M+M R+\sum_{i=1}^{r} \xi_{i} \frac{\partial M}{\partial x_{i}} .
$$

Agora, como primeira parte do próximo passo construímos o $(l+1)$ - jato de $\phi$. Consideremos o campo de vetores

$$
\delta=-\frac{\partial}{\partial y_{y_{k-l}}}+\sum_{i=1}^{N} \xi_{i} \frac{\partial}{\partial x_{i}}
$$

e introduzimos uma nova variável $t$. Definimos um automorfismo $\Phi$ de $\mathbb{K}[[\underline{x}, \underline{y}, t]]$ dado por

$$
\begin{aligned}
& a \longmapsto \sum_{\nu=1}^{\infty} \frac{1}{\nu !} \delta^{\nu}(a) t^{\nu} \text { para todo } a \in \mathbb{K}[[\underline{x}, \underline{y}]] \\
& t \longmapsto t .
\end{aligned}
$$

Em particular, temos

- $\Phi_{i}:=\Phi\left(x_{i}\right)=\sum_{\nu=1}^{\infty} \frac{1}{\nu !} \delta^{\nu}\left(x_{i}\right)(a) t^{\nu}=x_{i}+\xi_{i} t+$ termos de ordem alta em $t, 1 \leq i \leq r$

- $\Phi\left(y_{i}\right)=y_{i}$ para $1 \leq i \leq$ e $i \neq k-l$,

- $\Phi\left(y_{k-l}\right)=y_{k-l}-t$

Além disso, a derivada parcial em $t$ é dada por

$$
\frac{\partial \Phi}{\partial t}=\sum_{\nu=0}^{\infty} \frac{1}{\nu !} \delta^{\nu+1}\left(x_{i}\right) \nu t^{\nu}=\Phi\left(\delta\left(x_{i}\right)\right)=\xi_{i}\left(\underline{\Phi}, \ldots, y_{1}, \ldots, y_{k-l}, y_{k-l+1}, \ldots, y_{k}\right)
$$

onde $\underline{\Phi}=\left(\Phi_{1}, \ldots, \Phi_{r}\right)$. 
Usando esta igualdade, podemos analisar melhor a derivada de uma das entradas de $M$ em $t$, isto é, se $\underline{Y}=\left(y_{1}, \ldots, y_{k-l}, 0, \ldots, 0\right)$, então

$$
\begin{aligned}
\frac{\partial M_{i j}}{\partial t}(\underline{\Phi}, \underline{Y}) & =\left(\sum_{\nu=1}^{r} \frac{\partial M}{\partial x_{\nu}}(\underline{\Phi}, \underline{Y}) \frac{\partial \Phi_{\nu}}{\partial t}(\underline{x}, \underline{Y})\right)-\frac{\partial M_{i j}}{\partial y_{k-l}}(\underline{\Phi}, \underline{Y}) \\
& =\delta(M)_{i j}(\underline{\Phi}, \underline{Y})=-(L M+M R)_{i j}(\underline{\Phi}, \underline{Y}) .
\end{aligned}
$$

Finalmente, vamos construir $A^{(l+1)}$ e $B^{(l+1)}$. Sejam $K=K\left(x_{1}, \ldots, x_{r}, y_{1}, \ldots, y_{k-l}, t\right)$ $\in G L_{n}(\mathbb{K}[[\underline{x}, \underline{y}, t]])$ e $S=S\left(x_{1}, \ldots, x_{r}, y_{1}, \ldots, y_{k-l}, t\right) \in G L_{p}(\mathbb{K}[[\underline{x}, \underline{y}, t]])$ matrizes tais que

$$
\begin{aligned}
& K(\underline{x}, \underline{y}, 0)=I d_{n}, \\
& S(\underline{x}, \underline{y}, 0)=I d_{n+1}
\end{aligned}
$$

e o seguinte sistema de equações diferenciais

$$
\frac{(\partial K \widetilde{M} S)_{i j}}{\partial t}=-(L(\underline{\Phi}, \underline{Y}) K \widetilde{M} S+K \widetilde{M} S R(\underline{\Phi}, \underline{Y}))_{i j}, \forall 1 \leq i \leq k, 1 \leq j \leq p .
$$

seja satisfeito por

$$
\widetilde{M}=M\left(x_{1}, \ldots, x_{r}, y_{1}, \ldots, y_{k-l}, \underline{0}\right)
$$

Mas neste caso, basta resolver os dois seguintes sistemas de equações diferenciais

$$
\begin{gathered}
\frac{\partial K_{i j}}{\partial t}=-(L(\underline{\Phi}, \underline{Y}) K)_{i j}, \forall 1 \leq i \leq n, 1 \leq j \leq p \\
\frac{\partial S_{i j}}{\partial t}=-(S R(\underline{\Phi}, \underline{Y}))_{i j}, \forall 1 \leq i \leq n, 1 \leq j \leq p .
\end{gathered}
$$

com as mesmas condições iniciais e já resolvemos o sistema original. De fato, $\widetilde{M}$ independe de $t$ e o sistema original aparece multiplicando (2.9) à direita por $\widetilde{M} S,(2.10)$ à esquerda por $K \widetilde{M}$ e somando os sistemas resultantes.

A existência e unicidade das soluções $K$ e $S$ seguem diretamente escrevendo as entradas de $K$ e $S$ como séries de potências em $t$, e comparando os coeficientes.

\section{Teorema 2.3.1 (Critério Infinitesimal da Determinação Finita). Seja} $M \in \operatorname{Mat}_{(n, p)}\left(\mathbb{K}\left\{x_{1}, \ldots, x_{r}\right\}\right)$ um germe de matriz. Seja $k$ um inteiro positivo tal que

$$
\mathfrak{M}^{k+1} \operatorname{Mat}_{(n, p)}\left(\mathbb{K}\left\{x_{1}, \ldots, x_{r}\right\}\right) \subseteq \mathfrak{M}^{2}\left(\frac{\partial M}{\partial x_{1}}, \ldots, \frac{\partial M}{\partial x_{r}}\right)+\mathfrak{M} \operatorname{Im}(g) .
$$

Então, $M$ é $k$ - finitamente determinado. 


\section{Demonstração.}

Para provar o teorema, basta mostrar que qualquer perturbação $N$ de $M$ cujas entradas estão em $\mathfrak{M}^{k+1}$ é $\mathcal{G}$ - equivalente à $M$.

Consideremos $K \in \mathfrak{M}^{k+1} \operatorname{Mat}_{(n, p)}\left(\mathbb{K}\left\{x_{1}, \ldots, x_{r}\right\}\right)$. Seja

$$
M_{a}:=M+a K, 0 \leq a \leq 1
$$

Primeiro Passo: Vamos mostrar que para cada $M_{a}$, vale

$$
\mathfrak{M}^{k+1} M a t_{(n, p)}\left(\mathbb{K}\left\{x_{1}, \ldots, x_{r}\right\}\right) \subseteq \mathfrak{M}^{2}\left(\frac{\partial M_{a}}{\partial x_{1}}, \ldots, \frac{\partial M_{a}}{\partial x_{r}}\right)+\mathfrak{M} \operatorname{Im}(g) .
$$

Seja $\widetilde{K} \in \operatorname{Mat}_{(n, p)}\left(\mathbb{K}\left\{x_{1}, \ldots, x_{r}\right\}\right)$. Devemos encontrar $\xi_{i} \in \mathfrak{M}^{2}$ e matrizes quadradas $A$ e $B$, com entradas em $\mathfrak{M}$, de dimensões apropriadas tais que

$$
\widetilde{K}=\sum_{i=1}^{r} \xi_{i} \frac{\partial M_{a}}{\partial x_{i}}+A M_{a}+M_{a} B
$$

Uma vez que todas as entradas de $\widetilde{K}$ estão em $\mathfrak{M}^{k+1}$, sabemos que

$$
\widetilde{K}=\sum_{i=1}^{r} \widetilde{\xi}_{i} \frac{\partial M}{\partial x_{i}}+\widetilde{A} M+M \widetilde{B}
$$

onde $\widetilde{\xi}_{i} \in \mathfrak{M}^{2}$ e $\widetilde{A}_{i j}, \widetilde{B}_{i j} \in \widetilde{\mathfrak{M}}$ para todo $i, j$.

Portanto,

$$
\widetilde{K}=\sum_{i=1}^{r} \xi_{i} \frac{\partial M_{a}}{\partial x_{i}}+\widetilde{A} M_{a}+M_{a} \widetilde{B}-\left(\sum_{i=1}^{r} a \widetilde{\xi}_{i} \frac{\partial K}{\partial x_{i}}+a \widetilde{A} K+a K \widetilde{B}\right)
$$

Seja

$$
K^{(1)}=\left(\sum_{i=1}^{r} a \widetilde{\xi}_{i} \frac{\partial K}{\partial x_{i}}+a \widetilde{A} K+a K \widetilde{B}\right),
$$

então $K^{(1)} \in \mathfrak{M}^{k+2}$. Conseqüentemente, podemos reescrever $K^{(1)}$ como

$$
K^{(1)}=\sum_{i=1}^{r} \xi_{i}^{(1)} \frac{\partial K}{\partial x_{i}}+A^{(1)} M+M B^{(1)}
$$

onde $\xi_{i}^{(1)} \in \mathfrak{M}^{3}$ e $A_{i j}^{(1)}, B_{i j}^{(1)} \in \mathfrak{M}^{2}$. Substituindo em (2.11), obtemos

$$
\widetilde{K}=\sum_{i=1}^{r}\left(\widetilde{\xi}_{i}+\xi_{i}^{(1)}\right) \frac{\partial M_{a}}{\partial x_{i}}+\left(\widetilde{A}+A^{(1)}\right) M_{a}+M_{a}\left(\widetilde{B}+B^{(1)}\right)-\left(\sum_{i=1}^{r} a \widetilde{\xi}_{i}^{(1)} \frac{\partial K}{\partial x_{i}}+a A^{(1)} K+a K B^{(1)}\right) .
$$

Logo, tomando

$$
K^{(2)}=\left(\sum_{i=1}^{r} a \widetilde{\xi}_{i}^{(1)} \frac{\partial K}{\partial x_{i}}+a A^{(1)} K+a K B^{(1)}\right)
$$


segue que as entradas de $K^{(2)}$ estão em $\mathfrak{M}^{k+3}$.

Continuando o processo, podemos construir uma série formal $\xi_{i}$ e matrizes $A$ e $B$ com entradas no anel das séries de potências formais. Quando $\mathbb{K}=\mathbb{C}$, aplicando o Teorema de Aproximação de Artin, a afirmação se verifica no anel das séries de potências convergentes.

Segundo Passo: Mostremos que $M_{a}+t K \sim M_{a}$, para $t$ suficientemente pequeno.

Pelo primeiro passo já sabemos que

$$
K=\left(\sum_{i=1}^{r} \xi_{i}^{(1)} \frac{\partial M_{a}}{\partial x_{i}}+A M_{a}+M_{a} B\right) .
$$

Mas usando o Lemma (2.3.1), mostramos que a mesma afirmação ainda é válida se substituímos $M_{a}$ por $M_{a}+t K$. Para finalizar, basta repetir o argumento do primeiro passo, com a diferença que aparece uma potência alta na variável $t$ também em cada etapa. Aplicando (2.3.1) obtemos a exigência deste passo.

Terceiro Passo: Provemos que $M \sim M+K$.

Nos passos anteriores, vimos que para todo $a \in[0,1]$ existe uma vizinhança $U$ de $a$ tal que para todo $t \in U$, temos $M_{a}+t K \sim M_{a}$. Como o intervalo [0,1] é compacto, podemos cobri-lo por finitos $U^{\prime}$ s. Usando a transitividade da relação de equivalência $\sim$ obtemos

$$
M=M_{0} \sim M_{1}=M+K
$$

Exemplo 2.3.1. Consideremos a seqüência

$$
\left(\mathbb{C}^{4}, 0\right) \stackrel{A}{\longrightarrow} \operatorname{Mat}_{(2,3)}\left(\mathcal{O}_{4}\right) \stackrel{\Delta}{\longrightarrow}\left(\mathbb{C}^{3}, 0\right),
$$

onde $\Delta=\left(\Delta_{1}, \Delta_{2}, \Delta_{3}\right), \Delta_{i}, i=1,2,3$, é o menor $2 \times 2$ obtido da matriz $A$ retirando a $i$-ésima coluna de $A$ e $\mathcal{O}_{4}=\mathbb{C}\{x, y, z, w\}$. O ideal $I=\left\langle f_{1}, f_{2}, f_{3}\right\rangle$, onde $f_{i}=\Delta_{i} \circ A$, define uma variedade $\mathcal{V}(I)$ que é uma superfície em $\mathbb{C}^{4}$ que, em geral, não é uma interseção completa.

Por exemplo, consideremos o germe de matriz A, dada por

$$
A=\left(\begin{array}{ccc}
x & y & z \\
w & z^{k} & x
\end{array}\right), \forall k \geq 1
$$

Esta é a primeira forma normal da classificação de germes Cohen-Macalay de codimensão 2 obtida em [18]. 
Os geradores do espaço tangente são dados por

$$
\begin{aligned}
& \left(\begin{array}{lll}
1 & 0 & 0 \\
0 & 0 & 1
\end{array}\right),\left(\begin{array}{lll}
0 & 0 & 0 \\
1 & 0 & 0
\end{array}\right),\left(\begin{array}{ccc}
0 & 1 & 0 \\
0 & 0 & 0
\end{array}\right),\left(\begin{array}{ccc}
0 & 0 & 1 \\
0 & k z^{k-1} & 0
\end{array}\right),\left(\begin{array}{ccc}
x & 0 & 0 \\
0 & 0 & 0
\end{array}\right),\left(\begin{array}{lll}
0 & 0 & 0 \\
0 & w & 0
\end{array}\right), \\
& \left(\begin{array}{lll}
0 & 0 & x \\
0 & 0 & w
\end{array}\right),\left(\begin{array}{ccc}
y & 0 & 0 \\
0 & 0 & 0
\end{array}\right),\left(\begin{array}{ccc}
0 & 0 & 0 \\
0 & z^{k} & 0
\end{array}\right),\left(\begin{array}{ccc}
0 & 0 & y \\
0 & 0 & z^{k}
\end{array}\right),\left(\begin{array}{lll}
z & 0 & 0 \\
0 & 0 & 0
\end{array}\right),\left(\begin{array}{lll}
0 & 0 & 0 \\
0 & x & 0
\end{array}\right), \\
& \left(\begin{array}{lll}
0 & 0 & z \\
0 & 0 & x
\end{array}\right),\left(\begin{array}{ccc}
x & 0 & z \\
0 & 0 & 0
\end{array}\right),\left(\begin{array}{ccc}
w & 0 & x \\
0 & 0 & 0
\end{array}\right),\left(\begin{array}{lll}
0 & 0 & 0 \\
0 & y & z
\end{array}\right),\left(\begin{array}{lll}
0 & 0 & 0 \\
0 & z^{k} & x
\end{array}\right) .
\end{aligned}
$$

Aqui os quatro primeiros geradores vêm das derivadas $\frac{\partial M}{\partial x_{i}}$, com $x_{i}=x, y, z, w$. Neste caso, $\mathfrak{M}^{k+1} \operatorname{Mat}_{(2,3)}(\mathcal{O})$ está contido em $\mathfrak{M} \mathcal{T} \mathcal{G}_{(2,3)}$. A. Portanto, A é k-determinado.

Podemos relacionar a $\mathcal{G}$ - equivalência de matrizes com a noção de $\mathcal{K}_{\Delta}$-equivalência, onde $\Delta$ consiste da subvariedade formada pelas matrizes que não têm posto máximo ( [10]). É possível mostrar que sobre $\mathbb{C}$, as noções de $\mathcal{K}_{\Delta}$-equivalência e $\mathcal{G}$ - equivalência coincidem para germes finitamente determinados (ver [3]).

Observação: Os resultados desta seção também são verdadeiros para $\mathcal{P}=\mathcal{E}_{r}$. As provas são análogas, mas substituímos o Teorema de Artin pelo Lema de Nakayama.

\subsection{Determinação Finita: Critério Geométrico}

Vamos agora, caracterizar geometricamente os germes finitamente determinados no caso complexo. Inicialmente, observemos que a ação de $G L_{n}(\mathbb{C}) \times G L_{p}(\mathbb{C})$ em $\operatorname{Mat}_{(n, p)}(\mathbb{C})$ tem $n$ órbitas, se $n \leq p$, que determinam uma estratificação de $\operatorname{Mat}_{(n, p)}(\mathbb{C})$.

Para demonstrar a próxima proposição usamos argumentos análogos aos de [3]. Dessa forma, precisamos fixar algumas notações usando alguns elementos da teoria de feixes.

Consideremos $M$ um germe de matriz $n \times p$ com entradas em $\mathcal{O}_{r}$. Sejam $U$ e $V$ vizinhanças da origem em $\mathbb{C}^{r}$ e de $M(0)$ respectivamente. Denotamos por $\mathcal{O}(U)$ o feixe de funções analíticas em $U$ e por $\mathcal{O}(U, V)$ o feixe de aplicações de $U$ em $V$.

Teorema 2.4.1. (Critério Geométrico) Um elemento $M: \mathbb{C}^{r}, 0 \longrightarrow \operatorname{Mat}_{(n, p)}\left(\mathcal{O}_{r}\right)$ é $\mathcal{G}$-finitamente determinado se, e somente se, $M$ é transversal às órbitas da estratificação de $M a t_{(n, p)}(\mathbb{C})$ fora da origem. 


\section{Demonstração.}

Temos que um germe de matriz $M$ é $\mathcal{G}$-estável se

$$
\mathcal{O}_{r}\left\{M_{x_{(i)}}, R_{l k}, C_{i j}\right\}=\operatorname{Mat}_{(n, p)}\left(\mathcal{O}_{r}\right)
$$

$\underline{\text { Afirmação 1: }} M$ é $\mathcal{G}$-estável se, e somente se, as aplicações $\frac{\partial M}{\partial x_{i}}(0)$ são transversais à $G L_{p}(\mathbb{C}) \times G L_{n}(\mathbb{C})$-órbita de $M(0)$.

De fato, suponhamos que $M$ é $\mathcal{G}$-estável, então

$$
\operatorname{Mat}_{(n, p)}(\mathbb{C}) \subset \mathcal{T}_{e} \mathcal{G} \cdot M
$$

Assim,

$$
\mathcal{O}_{r}\left\{\frac{\partial M}{\partial x_{i}}(0), R_{l k}(0), C_{i j}(0)\right\}=\operatorname{Mat}_{(n, p)}(\mathbb{C}) .
$$

Observemos que (2.12) é uma condição de transversalidade entre a aplicação $M$ em 0 e a $G L_{n}(\mathbb{C}) \times G L_{p}(\mathbb{C})$-órbita de $M(0)$.

Reciprocamente, se $\frac{\partial M}{\partial x_{i}}(0), i=1, \ldots, r$, são transversais à $G L_{p}(\mathbb{C}) \times G L_{n}(\mathbb{C})$-órbita de $M(0)$, temos

$$
\mathcal{T}_{e} \mathcal{G} \cdot M+\mathfrak{M}_{m} \operatorname{Mat}_{(n, p)}\left(\mathcal{O}_{r}\right)=\operatorname{Mat}_{(n, p)}\left(\mathcal{O}_{r}\right) .
$$

Segue do Lema de Nakayama que $\operatorname{Mat}_{(n, p)}\left(\mathcal{O}_{r}\right)=\mathcal{T}_{e} \mathcal{G} \cdot M$, o que prova a afirmação 1.

Agora, consideremos o $\mathcal{O}(U)$-homomorfismo $t M: \operatorname{Mat}_{(n, p)}(U) \longrightarrow \mathcal{O}_{r}(U, V)$ e o $\mathcal{O}_{r}(U)$ submódulo $\mathcal{N}$ gerado por $R_{l k}$ e $C_{i j}$. Assim,

$$
N_{\mathcal{G}}(M)=\frac{\mathcal{O}_{r}(U, V)}{\left\{t M\left(\operatorname{Mat}_{(n, p)}(U)\right)+\mathcal{N}\right\}}
$$

é um feixe coerente. Então, usando o Teorema dos zeros de Hilbert (Nullstellensatz) para feixes coerentes, (ver [20]), a fibra de $N_{\mathcal{G}}(M)$ em 0 tem dimensão finita sobre $\mathbb{C}$ se, e somente se, existe uma vizinhança $U^{\prime} \subset U$ tal que $N_{\mathcal{G}}(M)$ se anule em $U^{\prime}-\{0\}$.

Logo,

$$
\left(N_{\mathcal{G}}(M)\right)_{y}=\frac{(\mathcal{O}(U, V))_{y}}{\left(\left\{t M\left(M a t_{(n, p)}(U)+N\right\}\right)_{y}\right.}=0
$$

para $y \in U^{\prime}$ e $y \neq 0$.

Mas a fibra em qualquer ponto pode ser identificada com o $\mathcal{G}$-espaço normal à órbita. Então, o germe $M$ é finitamente determinado se, e somente se, a condição de transversalidade é satisfeita fora da origem.

Como conseqüência do Critério Geométrico, Teorema 2.4.1, temos o seguinte resultado: 
Corolário 2.4.1. Seja $M$ uma matriz $n \times p$ com entradas no ideal maximal de $\mathcal{O}_{r}$ definindo uma singularidade isolada. Então, $M$ é finitamente determinada.

\section{Demonstração.}

Sejam $M: U \longrightarrow \operatorname{Mat}_{(n, p)}(\mathbb{C})$ um representante de $M$ definido em uma vizinhança da origem em $\mathbb{C}^{r}$ e $\left\{\Delta_{i}\right\}$ a estratificação de $M a t_{n, p}(\mathbb{C})$ determinada pela ação do grupo $G L_{p}(\mathbb{C}) \times G L_{n}(\mathbb{C})$. Observemos que como $M$ define uma singularidade isolada, então $M(U) \cap \Delta_{i}=\emptyset$ se $\Delta_{i} \neq$ $\{0\}$. Logo, $M$ é transversal a órbita da estratificação fora da origem. Portanto, pelo critério geométrico, $M$ é $\mathcal{G}$ - finitamente determinada.

Este resultado pode ser encontrado em [16], pág. 3998, porém a demonstração é feita usando argumentos diferentes.

\subsection{Subgrupos Geométricos}

Em [10], Damon definiu subgrupos especiais de $\mathcal{A}$ e $\mathcal{K}$ que podem ser vistos, de forma intuitiva, como subgrupos definidos por propriedades geométricas e que herdam propriedades naturais de $\mathcal{A}$ e $\mathcal{K}$. Estes grupos foram denominados subgrupos geométricos. Como conseqüência dos resultados obtidos por Damon em [10], podemos usar todas as técnicas usuais de teoria de singularidades, tais como o teorema de determinação finita e a teoria de desdobramentos, para estudar estes grupos.

O objetivo desta seção é mostrar que o grupo $\mathcal{G}_{(n, p)}$ é um subgrupo geométrico do grupo $\mathcal{K}$. Inicialmente, vamos introduzir a definição geral de subgrupos geométricos e posteriormente provaremos o resultado.

Seja $f_{0}:\left(\mathbb{K}^{r}, 0\right) \longrightarrow\left(\mathbb{K}^{p}, 0\right)$ um germe $\mathcal{C}^{\infty}$ ou real analítico se $\mathbb{K}=\mathbb{R}$ ou holomorfo quando $\mathbb{K}=\mathbb{C}$. Denotamos o conjunto destes germes por $\mathcal{C}_{r, p}$.

Um desdobramento a $q$ parâmetros de $f_{0}$, é um germe $f:\left(\mathbb{K}^{r+q}, 0\right) \longrightarrow\left(\mathbb{K}^{p+q}, 0\right)$, da forma $f(x, u)=(\bar{f}(x, u), u)$ tal que $\bar{f}(x, 0)=f_{0}(x)$. Para indicar o número de parâmetros do desdobramento, usamos a notação $(f, q)$. Denotamos por $\operatorname{Unf}(q)$ o conjunto de tais desdobramentos.

Seja $G$ um subgrupo de $\mathcal{K}$ que age em $\mathcal{F}$, um subespaço linear de $\mathcal{C}_{r, p}$. O grupo dos desdobramentos, $G_{u n}(q)$ de $G$, será um subgrupo de $\mathcal{K}_{u n}(q)$ agindo no espaço de desdobramentos $\mathcal{F}_{\text {un }}(q) \subseteq \operatorname{Unf}(q)$. Para $q=0$, temos exatamente $G$ e $\mathcal{F}$.

Definição 2.5.1. a) Sejam $(f, q)$ e $(g, m) \in \mathcal{F}_{\text {un }}$ desdobramentos de $f_{0}$, então uma $\mathcal{K}$-aplicação entre desdobramentos $\phi:(g, m) \longrightarrow(f, q)$ consiste em um desdobramento da identidade $H \in$ 
$\mathcal{K}_{\text {un }}(m)$ e uma mudança de parâmetros $\lambda:\left(\mathbb{K}^{m}, 0\right) \longrightarrow\left(\mathbb{K}^{q}, 0\right)$ tais que se

$$
H(x, y, v)=\left(\bar{h}(x, v), H_{1}(x, y, v), v\right)
$$

então

$$
H_{1}(x, \bar{g}(x, v), v)=\bar{f}(\bar{h}(x, v), \lambda(v)) .
$$

b) Um desdobramento $(f, q)$ de $f_{0}$ é $\mathcal{K}$-versal se para qualquer outro desdobramento $(g, m)$ de $f_{0}$ existe uma $\mathcal{K}$-aplicação de desdobramentos de $\phi:(g, m) \longrightarrow(f, q)$.

c) Dois desdobramentos $(f, m)$ e $(g, m)$ de $f_{0}$ são $\mathcal{K}$-equivalentes, se existem $\mathcal{K}$-aplicações de desdobramentos $\phi:(f, m) \longrightarrow(g, m)$ e $\psi:(g, m) \longrightarrow(f, m)$ tais que $\phi \circ \psi=I d$ e $\psi \circ \phi=I d$. Para estas equivalências, as aplicações entre os parâmetros $\lambda$ são germes de difeomorfismos. $O$ conjunto de tais equivalências será denotado por $\mathcal{K}_{e q}(m)$.

Como $G$ é um subgrupo de $\mathcal{K}$ podemos escrever a seguinte definição.

Definição 2.5.2. Sejam $(f, q)$ e $(g, r) \in \mathcal{F}_{\text {un }}$ desdobramentos de $f_{0}$, então uma G-aplicação entre desdobramentos $\phi:(g, r) \longrightarrow(f, q)$ é uma $\mathcal{K}$-aplicação entre desdobramentos.

Dessa forma, podemos definir $G$-desdobramentos versais e $G$-equivalência entre desdobramentos exatamente como na Definição (2.5.1), substituindo $\mathcal{K}$ por $G$. Desta forma, obtemos o grupo das equivalências $G_{e q}(r)$.

Em [10], J. Damon está interessado em descrever o espaço tangente de diversos grupos e germes de espaços como soma direta de módulos sobre germes de anéis. Muitos desses anéis, não têm a forma $\mathcal{P}$ para algum $\left(\mathbb{K}^{r}, 0\right)$. Contudo, existe uma classe extremamente útil de anéis, introduzida por Malgrange para a Teoria de Singularidades. Como nossa intenção é definir os subgrupo geométricos, faremos aqui apenas uma breve introdução desta classe. Para maiores detalhes ver [10].

Na descrição desta classe especial, além das categorias de germes suaves, reais analíticos e holomorfos, incluimos também a categoria dos germes de séries de potências formais. Nesta categoria, $\mathcal{C}_{x}$ denota $\mathbb{K}[[x]]$ o anel das séries de potências formais em $x=\left(x_{1}, \ldots, x_{r}\right)$.

Definição 2.5.3. Dizemos que uma álgebra A, diferenciável, analítica ou formal, consiste de uma $\mathbb{K}$-álgebra $A$ e um homomorfismo sobrejetivo de álgebras $\phi: \mathcal{C}_{x} \longrightarrow A$, onde $\mathcal{C}_{x}$ está na categoria apropriada. Denominamos tal álgebra de DA-álgebra.

Podemos, agora, definir os subgrupos geométricos. 
Definição 2.5.4. Seja $G$ um grupo agindo em $\mathcal{F}$, junto com o grupo de desdobramentos associados $G_{u n}$ agindo em $\mathcal{F}_{\text {un }}$. Dizemos que $G$ é um subgrupo geométrico de $\mathcal{K}$ se satisfaz as seguintes propriedades:

a) Naturalidade: Os grupos e os espaços de desdobramentos são naturais em relação ao pull-back. Isto é, se $f:\left(\mathbb{K}^{r} \times \mathbb{K}^{m}, 0\right) \longrightarrow\left(\mathbb{K}^{p+q}, 0\right)$ é um desdobramento de $f_{0}$ e $\lambda:\left(\mathbb{K}^{r}, 0\right) \longrightarrow\left(\mathbb{K}^{q}, 0\right)$, então o pull-back de $A$ via $\lambda, \lambda^{*} A$, é definido por $\lambda^{*} A(x, u)=$ $A(x, \lambda(u))$, para todo $x \in \mathbb{K}^{r}$ e $u \in \mathbb{K}^{m}$.

Dessa forma, a naturalidade significa que

$$
(\Phi, R, L) \in \mathcal{G}_{\text {un }}(q) \text { e } \lambda \in \mathcal{C}_{r, q} \Longrightarrow\left(\lambda^{*} \Phi, \lambda^{*} R, \lambda^{*} L\right) \in \mathcal{G}_{\text {un }}(q)
$$

\section{b) Estrutura do Espaço Tangente:}

i) Existe uma coleção de DA-subálgebras $\left\{R_{\alpha}\right\}$ de $\mathcal{C}_{x, y}$ tal que para cada $\mathbb{K}^{q}$, com coordenadas locais $u, \mathcal{T}_{e} G_{u n}$ e $\mathcal{T}_{e} \mathcal{F}_{\text {un }}$ são finitamente gerados como $R_{\alpha, u}$-módulos, com $\mathcal{T} G_{\text {un }}$ e $\mathcal{T} \mathcal{F}_{\text {un }}$ finitamente gerados como $R_{\alpha, u}$-submódulos e contendo, via naturalidade, $\mathcal{T}_{e} G$ e $\mathcal{T} \mathcal{F}$ como $\left\{R_{\alpha}\right\}$-submódulos. Além disso, para cada $f \in \mathcal{F}_{\text {un }}, R_{\alpha, u}$ torna-se um sistema de DA-álgebras sobre $\mathcal{C}_{u}$ tal que

$$
d \alpha_{f}: \mathcal{T}_{e} G_{\text {un }} \longrightarrow \mathcal{T}_{e} \mathcal{F}_{\text {un }}
$$

é um homomorfismo de $\left\{R_{\alpha, u}\right\}$-módulos.

ii) As seguintes aplicações naturais

$$
\begin{aligned}
& \frac{\mathcal{T}_{e} G_{u n}}{\mathfrak{M}_{u} \mathcal{T}_{e} G} \longrightarrow \mathcal{T}_{e} G \\
& \frac{\mathcal{T}_{e} \mathcal{F}_{u n}}{\mathfrak{M}_{u} \mathcal{T}_{e} \mathcal{F}_{u n}} \longrightarrow \mathcal{T}_{e} \mathcal{F}
\end{aligned}
$$

são isomorfismos de $\left\{R_{\alpha}\right\}$-módulos.

iii) Valem as inclusões

$$
\left\{\mathfrak{M}_{\alpha}\right\} \mathcal{T}_{e} \mathcal{F} \subset \mathcal{T} \mathcal{F} \quad e \quad\left\{\mathfrak{M}_{\alpha}\right\} \mathcal{T}_{e} G \subset \mathcal{T} G
$$

c) Aplicação Exponencial: A restrição da aplicação exponencial para $\mathcal{K}$, induz uma aplicação

$$
\exp : T_{e} G_{e q}(r) \longrightarrow G_{e q}(r+1)
$$

que envia elementos de $(T G)_{u}$ para germes de famílias a u-parâmetros em $G$. 
d) Condição de Filtração: $G_{u n}$ preserva a filtração $\left\{\left\{\mathfrak{M}^{l}\right\} \mathcal{F}_{u n}\right\}$ em $\mathcal{F}_{\text {un }}$ e induz uma ação no quociente $\frac{\mathcal{F}_{\text {un }}}{\left\{\mathfrak{M}^{l}\right\} \mathcal{F}_{\text {un }}}$ para todo $l \geq 0$.

Nosso próximo objetivo é mostrar que $\mathcal{G}_{(n, p)}$ é um subgrupo geométrico de $\mathcal{K}$. Para tanto, precisamos de algumas definições. Consideremos um germe de matriz $A:\left(\mathbb{C}^{r}, 0\right) \longrightarrow \operatorname{Mat}_{(n, p)}(\mathcal{P})$ e denotemos por $\mathcal{S}$ o conjunto das matrizes desta forma.

Definição 2.5.5. Um desdobramento a q parâmetros de um germe de matriz $A_{0} \in S$ é um germe

$$
A:\left(\mathbb{C}^{r} \times \mathbb{C}^{q}, 0\right) \longrightarrow \operatorname{Mat}_{(n, p)}(\mathcal{P}),
$$

onde $x=\left(x_{1}, \ldots x_{r}\right), y=\left(y_{1}, \ldots y_{q}\right), \mathcal{P}=\mathcal{O}_{r+q}$ ou $\mathcal{E}_{r+q}$, e $A(x, 0)=A_{0}(x)$.

Denotaremos por $\mathcal{S}_{\text {un }}$ o conjunto dos desdobramentos dos elementos de $\mathcal{S}$. O grupo $\mathcal{G}_{(n, p)}=$ $\mathcal{R} \times G L_{n}(\mathcal{P}) \times G L_{p}(\mathcal{P})$ agindo no espaço das matrizes da seguinte forma

$$
\begin{aligned}
& \mathcal{G}_{(n, p)} \times \operatorname{Mat}_{(n, p)}(\mathcal{P}) \longrightarrow \operatorname{Mat}_{(n, p)}(\mathcal{P}) \\
& \left(\left(\Phi_{0}, R_{0}, L_{0}\right), A\right) \longmapsto L_{0}^{-1}\left(M_{0} \circ \Phi_{0}\right) R_{0}
\end{aligned}
$$

Correspondente ao grupo $\mathcal{G}_{(n, p)}$, podemos construir o grupo dos desdobramentos, denotado por

$\mathcal{G}_{\text {un }}(q)=\left\{(\Phi, R, L) \in\left(\mathcal{R} \times G L_{n}(\mathcal{P}) \times G L_{p}(\mathcal{P})\right)_{r+q}:(\Phi, R, L)\right.$ são deformações de $\left.\left(\Phi_{0}, R_{0}, L_{0}\right)\right\}$.

Podemos definir a ação de $\mathcal{G}_{\text {un }}(q)$ em $\mathcal{S}_{\text {un }}$ de maneira análoga a que $\mathcal{G}_{(p, n)}$ age em $\mathcal{S}$, isto é,

$$
\begin{aligned}
& \mathcal{G}_{\text {un }}(q) \times \mathcal{S}_{\text {un }} \longrightarrow \mathcal{S}_{\text {un }} \\
& ((\Phi, R, L), M) \longmapsto L^{-1}(M \circ \Phi) R .
\end{aligned}
$$

Conseqüentemente, $L^{-1}(M \circ \Phi) R$ é um desdobramento de $L_{0}^{-1}\left(M_{0} \circ \Phi_{0}\right) R$.

Proposição 2.5.1. O grupo $\mathcal{G}_{(n, p)}$ é um subgrupo geométrico de $\mathcal{K}$.

\section{Demonstração.}

Mostremos que $\mathcal{G}_{(n, p)}$ satisfaz às quatro propriedades que aparecem na definição (2.5.5).

a) Naturalidade.

Seja $A:\left(\mathbb{K}^{r} \times \mathbb{K}^{m}, 0\right) \longrightarrow \operatorname{Mat}_{(n, p)}(\mathcal{P})$ um desdobramento de $A_{0}$ e $\lambda:\left(\mathbb{K}^{m}, 0\right) \longrightarrow\left(\mathbb{K}^{q}, 0\right)$, então o pull-back de $A$ via $\lambda, \lambda^{*} A$, é definido por $\lambda^{*} A(x, u)=A(x, \lambda(u))$, para todo $x \in \mathbb{K}^{r} \mathrm{e}$ $u \in \mathbb{K}^{m}$. 
Dessa forma, a naturalidade significa que dado um germe $\lambda \in \mathcal{C}_{m, q}$ como acima, temos

$$
\left(\lambda^{*} \Phi, \lambda^{*} R, \lambda^{*} L\right) \in \mathcal{G}_{u n}(q)
$$

Sabemos que

$$
\lambda^{*} \Phi(x, v)=\Phi(x, \lambda(v))
$$

com

$$
\lambda^{*} \Phi(x, 0)=\Phi(x, \lambda(0))=\Phi(x, 0)=\Phi_{0}(x) \in \mathcal{R}
$$

Segue do Teorema da Função Inversa que existe uma vizinhança da origem tal que $\lambda^{*} \Phi$ é difeomorfismo. Portanto, $\lambda^{*} \Phi \in \mathcal{R}_{r+m}$.

Da mesma forma, temos

$$
\lambda^{*} R(x, v)=R(x, \lambda(v))
$$

com

$$
\lambda^{*} R(x, 0)=R(x, \lambda(0))=R(x, 0)=R_{0}(x) \in G L_{n}(\mathcal{P}) .
$$

Logo, na origem $\lambda^{*} R$ é uma matriz invertível. Como o determinante é uma função contínua segue que existe uma vizinhança da origem tal que $\lambda^{*} R$ é invertível, isto é, $\lambda^{*} R \in G L_{n}(\mathcal{P})$.

Analogamente, mostramos que $\lambda^{*} L \in G L_{p}(\mathcal{P})$. Portanto, $\mathcal{G}$ satisfaz à propriedade de naturalidade.

b) Espaço Tangente.

As expressões do espaço tangente e do espaço tangente estendido são

$$
\begin{aligned}
& T \mathcal{G} M_{0}=\mathfrak{M}\left\{\frac{\partial M_{0}}{\partial x_{i}}\right\}+\mathcal{P}\left\{A M_{0}+M_{0} B: A \in G L_{n}(\mathcal{P}), B \in G L_{p}(\mathcal{P})\right\} \\
& T \mathcal{G}_{e} M_{0}=\mathcal{P}\left\{\frac{\partial M_{0}}{\partial x_{i}}\right\}+\mathcal{P}\left\{A M_{0}+M_{0} B: A \in G L_{n}(\mathcal{P}), B \in G L_{p}(\mathcal{P})\right\}
\end{aligned}
$$

O espaço tangente estendido é um $\mathcal{P}$-módulo finitamente gerado e o espaço tangente é finitamente gerado sobre o sistema de módulos $\{\mathfrak{M}, \mathcal{P}\}$.

Além disso, se definimos

$$
\begin{gathered}
\alpha: \mathcal{G}_{(n, p)} \times \operatorname{Mat}_{(n, p)}(\mathcal{P}) \longrightarrow \operatorname{Mat}_{(n, p)}(\mathcal{P}) \\
\left(\left(\Phi_{0}, R_{0}, L_{0}\right), M_{0}\right) \longmapsto L_{0}^{-1}\left(M_{0} \circ \Phi\right) R_{0},
\end{gathered}
$$

fixado $M_{0} \in S$, temos a aplicação órbita

$$
\begin{aligned}
& \alpha_{M_{0}}: \mathcal{G}_{(n, p)} \longrightarrow \operatorname{Mat}_{(n, p)}(\mathcal{P}) \\
& \left(\Phi_{0}, R_{0}, L_{0}\right) \longmapsto L_{0}^{-1}\left(M_{0} \circ \Phi\right) R_{0} .
\end{aligned}
$$


Portanto,

$$
\begin{aligned}
d \alpha_{M_{0}}: T \mathcal{G} M_{0} & \longrightarrow \operatorname{TMat}_{(n, p)}(\mathcal{P}) \\
(\xi, A, B) & \longmapsto \xi\left(M_{0}\right)+A M_{0}+M_{0} B
\end{aligned}
$$

onde $\xi(M)$ indica as derivadas de $M$ em relação à $x_{i}$. Note que $d \alpha_{M_{0}}$ é homomorfismo de módulos.

A restrição de $d \alpha_{M_{0}}$ ao somando $\mathcal{P} \frac{\partial}{\partial x_{i}}$, é um $\mathcal{P}$-homomorfismo tal que

$$
\frac{\partial}{\partial x_{i}} \longmapsto \frac{\partial M_{0}}{\partial x_{i}}
$$

Da mesma forma, a restrição de $d \alpha_{M_{0}}$ à $\operatorname{Mat}_{n}(\mathcal{P}) \times M a t_{p}(\mathcal{P})$ é um homomorfismo de módulos tal que

$$
(A, B) \longmapsto A M_{0}+M_{0} B
$$

Para finalizar, observe que

$$
\frac{T_{e} \mathcal{G}_{\text {un }}}{\mathfrak{M}_{u} T_{e} \mathcal{G}_{\text {un }}} \cong \mathfrak{M} \theta_{M_{0}} \times \operatorname{Mat}_{n}(\mathcal{P}) \times \operatorname{Mat}_{p}(\mathcal{P})=T_{e} \mathcal{G} M
$$

$\mathrm{e}$

$$
\frac{T_{e} \mathcal{S}_{u n}}{\mathfrak{M} T_{e} \mathcal{S}_{u n}} \cong T_{e} \mathcal{S}_{u n}
$$

Portanto, $\mathcal{G}$ satisfaz à propriedade do espaço tangente.

c) Exponencial

Consideremos a restrição da aplicação exponencial em $\mathcal{K}$

$$
\exp : T_{e} \mathcal{G}_{\text {un }}(q) \longrightarrow T_{e} \mathcal{G}_{\text {un }}(q+1)
$$

onde $\exp (\tau)=\psi, \psi=(\Phi, R, L)$ e $\frac{\partial \Phi_{t}}{\partial t}=\Phi^{\prime} \circ \Phi_{t}$ e $\Phi_{0}=I d \in \mathcal{G}_{e q}(e)$ assim, esta propriedade segue diretamente para o subgrupo $\mathcal{G}$.

Analogamente, a propriedade da filtração segue diretamente do fato que $\mathcal{G}_{(n, p)}$ é subgrupo de $\mathcal{K}$.

\subsection{Propriedades da $\mathcal{G}$ - Equivalência}

O objetivo desta seção é demonstrar o Teorema 2.6.1 que estabelece equivalências entre a $\mathcal{G}$ determinação finita de matrizes $n \times(n+1)$ com entradas em $\mathcal{O}_{r}$ e a condição geométrica que a 
variedade $X=f^{-1}(0)$ tem singularidade isolada na origem. O resultado análogo para o grupo $\mathcal{K}$ foi demonstrado por T. Gaffney em [20].

Seja $M=\left(m_{s u}\right)$ uma matriz $n \times(n+1)$, com entradas no ideal maximal de $\mathcal{P}$ com $n>1 \mathrm{e}$ $r>1$. Denotamos por $f=\left(f_{1}, \ldots, f_{n+1}\right)$ o ideal gerado pelos menores $n \times n$ da matriz $M$. O índice de cada $f_{i}$ indica a coluna retirada de $M$ para calcular o menor.

Seja

$$
J M(X)=\left(\begin{array}{cccc}
\frac{\partial f_{1}}{\partial x_{1}} & \frac{\partial f_{1}}{\partial x_{2}} & \cdots & \frac{\partial f_{1}}{\partial x_{r}} \\
\frac{\partial f_{2}}{\partial x_{1}} & \frac{\partial f_{2}}{\partial x_{2}} & \cdots & \frac{\partial f_{2}}{\partial x_{r}} \\
\vdots & \vdots & \ddots & \vdots \\
\frac{\partial f_{n+1}}{\partial x_{1}} & \frac{\partial f_{n+1}}{\partial x_{2}} & \cdots & \frac{\partial f_{n+1}}{\partial x_{r}}
\end{array}\right)
$$

a matriz jacobiana de $f$. Como $X$ não é uma intersecção completa, existem relações entre as funções componentes de $f$. Assim, o posto maximal de $J M(X)$ é dado por $d \leq \min \{n, r\}$.

Consideremos os seguintes conjuntos

$$
\begin{aligned}
& L=\left\{\left\{i_{1}, \ldots, i_{d}\right\} \in \mathbb{N}^{d} \mid 1 \leq i_{\tau} \leq n+1 \text { e } i_{\alpha} \neq i_{\beta} \text { se } \alpha \neq \beta\right\} \\
& V=\left\{\left\{j_{1}, \ldots, j_{d}\right\} \in \mathbb{N}^{d} \mid 1 \leq j_{\tau} \leq r \text { e } j_{\alpha} \neq j_{\beta} \text { se } \alpha \neq \beta\right\}
\end{aligned}
$$

Fixemos algumas notações:

a) $E_{i j}$ representa a matriz $n \times(n+1)$ que tem 1 na posição $(i, j)$ e zero em qualquer outro lugar;

b) Sejam $i=\left(i_{1}, \ldots, i_{d}\right) \in L$ e $j=\left(j_{1}, \ldots, j_{d}\right) \in V$. Denotamos por $\Delta_{i}^{j}$ o determinante da matriz obtida com as linhas $i_{1}, \ldots, i_{d}$ e as colunas $j_{1}, \ldots, j_{d}$ de $J M(X)$.

c) No caso particular dos menores $2 \times 2$ da matriz $J M(X)$, usaremos a seguinte notação:

$$
\Delta_{(\gamma, \nu)}^{(j, t)}=\frac{\partial f_{j}}{\partial x_{\gamma}} \frac{\partial f_{t}}{\partial x_{\nu}}-\frac{\partial f_{j}}{\partial x_{\nu}} \frac{\partial f_{t}}{\partial x_{\gamma}}
$$

$\operatorname{com} 1 \leq j, t \leq n+1,1 \leq \gamma, \nu \leq r, j \neq t$ e $\gamma \neq \nu$

d) Denotamos por $J_{f}$ o ideal gerado pelos menores $d \times d$ da matriz jacobiana de $f$, isto é, $J_{f}=\left\langle\Delta_{i}^{j}: i \in L\right.$ e $\left.j \in V\right\rangle$.

e) Seja $M^{j}$ a matriz $n \times n$, obtida retirando-se a $j$-ésima coluna de $M$. Indicamos por $\operatorname{cof}^{j}\left(m_{s u}\right)$ o cofator do elemento $m_{s u}$ em $M^{j}$. 
f) Analogamente, $M_{k i}^{j}$ é a matriz $(n-1) \times(n-1)$ obtida retirando-se de $M^{j}$ a $k$ - ésima linha e a $i$ - ésima coluna. Denotamos por $\operatorname{cof}_{k i}^{j}\left(m_{s u}\right)$ o cofator do elemento $m_{s u}$ em $M_{k i}^{j}$.

Na próxima proposição, mostramos que as matrizes $f_{j} E_{k l}$ pertencem ao espaço tangente à $M$ para todo $1 \leq l, j \leq n+1$ e $1 \leq k \leq n$.

Proposição 2.6.1. Seja $M$ uma matriz, $n \times(n+1)$, com entradas no ideal maximal de $\mathbb{C}\left\{x_{1}, \ldots, x_{r}\right\}$. Então, $f_{j} E_{k l} \in T \mathcal{G} M$, para $1 \leq l, j \leq n+1$ e $1 \leq k \leq n$.

\section{Demonstração.}

Se $M=\left(m_{u s}\right)$, podemos escrever cada $f_{j}$ da seguinte forma:

$$
f_{j}=\sum_{s \neq j} m_{k s} \operatorname{cof}^{j}\left(m_{k s}\right) \text {. }
$$

Consideremos a matriz, $n \times(n+1), A=\left(a_{u s}\right)$ definida por

$$
A=\sum_{s \neq j} \operatorname{cof}^{j}\left(m_{k s}\right) C_{l s} \in T \mathcal{G} M
$$

Então, temos:

i) $a_{u s}=0$, se $s \neq l$;

ii) $a_{k l}=\sum_{s \neq l} m_{k s} \operatorname{cof}^{k}\left(m_{k s}\right)=f_{j}$;

iii) $a_{u l}=\sum_{s \neq j} m_{u s} \operatorname{cof}^{j}\left(m_{k s}\right)$, para $u \neq k$.

Observemos que $\sum_{s \neq j} m_{u s} \operatorname{cof}^{j}\left(m_{k s}\right)=0$, para $u \neq k$, pois é a expressão do determinante de uma matriz que tem duas colunas iguais a $s$ - ésima coluna de $M$. Logo, $A=f_{j} E_{k l}$ e obtemos o resultado.

Nosso próximo objetivo é mostrar que os menores $d \times d$ da matriz jacobiana de $f$ estão no espaço tangente a $M$. Para isso, precisamos provar alguns resultados preliminares:

Proposição 2.6.2. Seja $M=\left(m_{u s}\right)$ uma matriz $n \times(n+1)$ com entradas no ideal maximal de $\mathcal{P}$. Então,

$$
\operatorname{cof}^{j}\left(m_{i l}\right)=(-1)^{\alpha} \operatorname{cof}^{l}\left(m_{i j}\right)
$$

onde $l \neq j$ e

$$
\alpha=\left\{\begin{array}{l}
l-j+1, \text { se } l<j \\
l-j-1, \text { se } l>j
\end{array}\right.
$$

$1 \leq l, j \leq n+1$ e $1 \leq i \leq n$. 


\section{Demonstração.}

Inicialmente, seja $l<j$. Então,

$$
\operatorname{cof}^{j}\left(m_{i l}\right)=(-1)^{i+l} \operatorname{det}\left(M_{i l}^{j}\right)
$$

onde $M_{i l}^{j}$ é a matriz obtida retirando-se a linha $i$ e as colunas $j$ e $l$ da matriz $M$. Por outro lado,

$$
\operatorname{cof}^{l}\left(m_{i j}\right)=(-1)^{i+j-1} \operatorname{det}\left(M_{i j}^{l}\right)=(-1)^{i+j-1} \operatorname{det}\left(M_{i l}^{j}\right) .
$$

$\operatorname{Logo}, \operatorname{cof}^{j}\left(m_{i l}\right)=(-1)^{l-j+1} \operatorname{cof}^{l}\left(m_{i j}\right)$.

Para $l>j$, procedemos analogamente e obtemos $\operatorname{cof}^{j}\left(m_{i l}\right)=(-1)^{l-j-1} \operatorname{cof}^{l}\left(m_{i j}\right)$.

Proposição 2.6.3. As matrizes

$$
\frac{\partial f_{j}}{\partial x_{\gamma}} E_{k l}+(-1)^{l-j+1} \frac{\partial f_{l}}{\partial x_{\gamma}} E_{k j} \in T \mathcal{G} M
$$

para $j \neq l, 1 \leq j, l \leq n+1,1 \leq \gamma \leq r$ e $1 \leq k \leq n$.

\section{Demonstração.}

Suponhamos, sem perda de generalidade, que $l<j$. Vamos mostrar este resultado em cinco passos:

Primeiro Passo: Consideremos a matriz $A=\left(a_{q v}\right)$ definida por

$$
A=\frac{\partial M}{\partial x_{\gamma}} \operatorname{cof}^{j}\left(m_{k l}\right)+\sum_{i \neq j} \frac{\partial \operatorname{cof}^{j}\left(m_{k i}\right)}{\partial x_{\gamma}} C_{l i}+\sum_{u \neq j, l}(-1)^{\alpha} \frac{\partial m_{k i}}{\partial x_{\gamma}}\left(\sum_{i \neq k} R_{k s} \operatorname{cof}_{k i}^{j}\left(m_{s l}\right)\right)
$$

onde

$$
\alpha=\left\{\begin{array}{l}
k+i, \text { se } i<j \\
k+i-1, \text { se } i>j .
\end{array}\right.
$$

Vamos olhar para cada entrada da matriz $A$.

i) Na entrada $(k, j)$, temos

$$
\begin{aligned}
a_{k j} & =\frac{\partial m_{k j}}{\partial x_{\gamma}} \operatorname{cof}^{j}\left(m_{k l}\right)+\sum_{i \neq j}(-1)^{\alpha} \frac{\partial m_{k i}}{\partial x_{\gamma}}\left(\sum_{u \neq j, k} m_{u j} \operatorname{cof}_{k i}^{j}\left(m_{u l}\right)\right) \\
& =(-1)^{l-j+1}\left(\frac{\partial m_{k j}}{\partial x_{\gamma}} \operatorname{cof}^{l}\left(m_{k j}\right)+\sum_{i \neq j}(-1)^{\alpha} \frac{\partial m_{k i}}{\partial x_{\gamma}}\left(\sum_{u \neq j, k} m_{u j} \operatorname{cof}_{k i}^{l}\left(m_{u j}\right)\right)\right) \\
& =(-1)^{l-j+1}\left(\frac{\partial f_{l}}{\partial x_{\gamma}}-\sum_{i \neq l} \frac{\partial \operatorname{cof}^{l}\left(m_{k i}\right)}{\partial x_{\gamma}} m_{k i}\right) .
\end{aligned}
$$


ii) Na entrada $(k, l)$, temos

$$
\begin{aligned}
a_{k l} & =\frac{\partial m_{k l}}{\partial x_{\gamma}} \operatorname{cof}^{j}\left(m_{k l}\right)+\sum_{i \neq j} \frac{\partial \operatorname{cof}^{j}\left(m_{k i}\right)}{\partial x_{\gamma}} m_{k i}+\sum_{i \neq j, l}(-1)^{\alpha} \frac{\partial m_{k i}}{\partial x_{\gamma}}\left(\sum_{u \neq j, k} m_{u l} \operatorname{cof}_{k i}^{j}\left(m_{u l}\right)\right) \\
& =\sum_{i \neq j} \frac{\partial \operatorname{cof}^{j}\left(m_{k i}\right)}{\partial x_{\gamma}} m_{k i}+\sum_{i \neq j} \frac{\partial m_{k i}}{\partial x_{\gamma}} \operatorname{cof}^{j}\left(m_{k i}\right)=\frac{\partial f_{j}}{\partial x_{\gamma}} .
\end{aligned}
$$

iii) Se $t \neq l, j$, temos na entrada $(k, t)$,

$$
a_{k t}=\frac{\partial m_{k t}}{\partial x_{\gamma}} \operatorname{cof}^{j}\left(m_{k l}\right)+\sum_{i \neq j}(-1)^{\alpha} \frac{\partial m_{k i}}{\partial x_{\gamma}}\left(\sum_{u \neq j, k} m_{u t} \operatorname{cof}_{k i}^{j}\left(m_{u l}\right)\right) .
$$

Notemos que

$$
\sum_{u \neq k} m_{u t} \operatorname{cof}_{k i}^{j}\left(m_{u l}\right)=0
$$

para $i \neq t$, pois esta é a expressão do determinante de uma matriz $(n-1) \times(n-1)$ que tem duas colunas iguais a $l$-ésima coluna da matriz $M$. Assim,

$$
a_{k t}=\frac{\partial m_{k t}}{\partial x_{\gamma}} \operatorname{cof}^{j}\left(m_{k l}\right)+(-1)^{k+t} \frac{\partial m_{k t}}{\partial x_{\gamma}}\left(\sum_{u \neq j, k} m_{u t} \operatorname{cof}_{k t}^{j}\left(m_{u l}\right)\right) .
$$

Então,

a) Se $l<t$, então

$$
\frac{\partial m_{k t}}{\partial x_{\gamma}}\left(\operatorname{cof}^{j}\left(m_{k l}\right)+(-1)^{k+l+1} \sum_{u \neq j, k} m_{u t} \operatorname{cof}_{k l}^{j}\left(m_{u t}\right)\right)=\frac{\partial m_{k t}}{\partial x_{\gamma}}\left(\operatorname{cof}^{j}\left(m_{k l}\right)-\operatorname{cof}^{j}\left(m_{k l}\right)\right)=0 .
$$

b) Se $l>t$, então

$$
\frac{\partial m_{k t}}{\partial x_{\gamma}}\left(\operatorname{cof}^{j}\left(m_{k l}\right)+(-1)^{k+l-1} \sum_{u \neq j, k} m_{u t} \operatorname{cof}_{k l}^{j}\left(m_{u t}\right)\right)=\frac{\partial m_{k t}}{\partial x_{\gamma}}\left(\operatorname{cof}^{j}\left(m_{k l}\right)-\operatorname{cof}^{j}\left(m_{k l}\right)\right)=0 .
$$

Portanto, $a_{k t}=0$, para todo $t \neq l, j$.

iv) Seja $q \neq k$, então na entrada $(q, l)$ temos:

$$
a_{q l}=\frac{\partial m_{q l}}{\partial x_{\gamma}} \operatorname{cof}^{j}\left(m_{k l}\right)+\sum_{i \neq j} \frac{\partial \operatorname{cof}^{j}\left(m_{k i}\right)}{\partial x_{\gamma}} m_{q i}
$$

Podemos escrever $\operatorname{cof}^{j}\left(m_{k i}\right)=(-1)^{\beta} \sum_{t \neq i, j} m_{q t} \operatorname{cof}_{k i}^{j}\left(m_{q t}\right)$, onde $\beta=\left\{\begin{array}{l}k+i, \text { se } i<j \\ k+i-1, \text { se } i>j\end{array}\right.$ 
Então,

$$
a_{q l}=\frac{\partial m_{q l}}{\partial x_{\gamma}} \operatorname{cof}^{j}\left(m_{k l}\right)+\sum_{i \neq j}(-1)^{\beta}\left(\sum_{t \neq i, j} \frac{\partial m_{q t}}{\partial x_{\gamma}} \operatorname{cof}_{k i}^{j}\left(m_{q t}\right)+m_{q t} \frac{\partial \operatorname{cof}_{k i}^{j}\left(m_{q t}\right)}{\partial x_{\gamma}}\right) m_{q i} .
$$

Para cada $t$ fixo, a segunda parcela da soma anterior se torna:

$$
\sum_{i \neq j, t}(-1)^{\beta}\left(\frac{\partial m_{q t}}{\partial x_{\gamma}} \operatorname{cof}_{k i}^{j}\left(m_{q t}\right)+m_{q t} \frac{\partial \operatorname{cof}_{k i}^{j}\left(m_{q t}\right)}{\partial x_{\gamma}}\right) m_{q i}
$$

Sem perda de generalidade, vamos supor que $t<j$. Tomando a primeira parcela de 2.15, temos

$$
\begin{aligned}
\sum_{i \neq j, t}(-1)^{\beta} m_{q i} \operatorname{cof}_{k i}^{j}\left(m_{q t}\right)=\sum_{i<j}(-1)^{k+i} m_{q i} \operatorname{cof}_{k i}^{j}\left(m_{q t}\right)+\sum_{i>j}(-1)^{k+i-1} m_{q i} \operatorname{cof}_{k i}^{j}\left(m_{q t}\right)= \\
=(-1)^{k+t}\left(\sum_{t<i<j}(-1)^{q+i} m_{q i} \operatorname{det}\left(M_{k t, q i}^{j}\right)+\sum_{i<t<j}(-1)^{q+i-1} m_{q i} \operatorname{det}\left(M_{k t, q i}^{j}\right)\right. \\
\left.+\sum_{t<j<i}(-1)^{q+i} m_{s i} \operatorname{det}\left(M_{k t, q i}^{j}\right)\right)=(-1)^{k+t+1}\left(\sum_{i \neq j, t}(-1)^{\varsigma} m_{q i} \operatorname{det}\left(M_{k t, q i}^{j}\right)\right),
\end{aligned}
$$

onde $\varsigma$ é o valor de $s+i$ na matriz $M_{k l, q i}^{j}$. Logo,

$$
\frac{\partial m_{q t}}{\partial x_{\gamma}} \sum_{i \neq j, t}(-1)^{\beta} \operatorname{cof}_{k i}^{j}\left(m_{q t}\right)=-\frac{\partial m_{q t}}{\partial x_{\gamma}} \operatorname{cof}^{j}\left(m_{k t}\right) .
$$

Em particular, se $t=l$, temos $\frac{\partial m_{q t}}{\partial x_{\gamma}} \sum_{i \neq j, l}(-1)^{\beta} \operatorname{cof}_{k i}^{j}\left(m_{q l}\right)=-\frac{\partial m_{q l}}{\partial x_{\gamma}} \operatorname{cof} f^{j}\left(m_{k l}\right)$.

Substituindo na expressão 2.14, temos

$$
a_{q l}=\sum_{t \neq j, l}-\frac{\partial m_{q t}}{\partial x_{\gamma}} \operatorname{cof}^{j}\left(m_{k t}\right)+\sum_{i \neq j}(-1)^{\beta}\left(\sum_{t \neq j, i} m_{q t} \frac{\partial \operatorname{cof}_{k i}^{j}\left(m_{q t}\right)}{\partial x_{\gamma}}\right) m_{q i} .
$$

\section{Afirmação:}

$$
\sum_{i \neq j}(-1)^{\beta}\left(\sum_{t \neq j, i} m_{q t} \frac{\partial \operatorname{cof}_{k i}^{j}\left(m_{q t}\right)}{\partial x_{\gamma}}\right) m_{q i}=0 .
$$

De fato, fixando $i=t_{0}$ e supondo, sem perda de generalidade, $t_{0}<j$, temos

$$
(-1)^{k+t_{0}} m_{q t_{0}} \sum_{t \neq j, t_{0}} \frac{\partial \operatorname{cof}_{k t_{0}}^{j}\left(m_{q t}\right)}{\partial x_{\gamma}} m_{q t}
$$

Agora, fazendo $t=t_{0}$ segue que $i \neq t_{0}$, então

$$
\sum_{i \neq j, t}(-1)^{\beta} m_{q t_{0}} \frac{\partial \operatorname{cof}_{k i}^{j}\left(m_{q t_{0}}\right)}{\partial x_{\gamma}} m_{q i}=(-1)^{k+t_{0}+1} m_{q t_{0}}\left(\sum_{i \neq j, t_{0}} \frac{\partial \operatorname{coj}_{k t_{0}}^{j}\left(m_{q i}\right)}{\partial x_{\gamma}} m_{q i}\right)
$$


Assim, parcelas diferentes da soma 2.16 são iguais, mas com sinais contrários, o que prova a afirmação.

Logo,

$$
a_{q l}=\sum_{t \neq j, l}-\frac{\partial m_{q t}}{\partial x_{\gamma}} \operatorname{cof}^{j}\left(m_{k t}\right)
$$

v) Na entrada $(q, v)$, para $v \neq l$ e $q \neq k$ :

$$
a_{q v}=\frac{\partial m_{q v}}{\partial x_{\gamma}} \operatorname{cof}^{j}\left(m_{k l}\right) .
$$

Segundo Passo: Consideremos a matriz dada por:

$$
B=(-1)^{j-l-1} \sum_{i \neq l} \frac{\partial \operatorname{cof}^{j}\left(m_{k i}\right)}{\partial x_{r}} C_{j l}
$$

Então,

i) $b_{u s}=0$, se $s \neq j$;

ii) $b_{u j}=(-1)^{j-l-1} \sum_{i \neq l} \frac{\partial \operatorname{cof}^{j}\left(m_{k i}\right)}{\partial x_{r}} m_{u i}$

Terceiro Passo: Consideremos a matriz, $n \times n+1, C=\left(c_{q v}\right)$ dada por $C=A+B$. Então,

i) $c_{k j}=(-1)^{l-j+1} \frac{\partial f_{l}}{\partial x_{\gamma}}$;

ii) $c_{k l}=\frac{\partial f_{j}}{\partial x_{\gamma}}$;

iii) $c_{k t}=0$, para $t \neq l, j$;

iv) $c_{q l}=-\sum_{t \neq j, l} \frac{\partial m_{q t}}{\partial x_{\gamma}} \operatorname{cof}^{j}\left(m_{k t}\right)$, para $q \neq k$.

v) $c_{q j}=\frac{\partial m_{q j}}{\partial x_{\gamma}} \operatorname{cof}^{j}\left(m_{k l}\right)+(-1)^{j-l-1} \sum_{i \neq l} \frac{\partial \operatorname{cof}^{j}\left(m_{k i}\right)}{\partial x_{r}} m_{u i}$, para $q \neq k$. Procedendo de forma análoga ao feito no item iv) do primeiro passo, é possível mostrar que

$$
c_{q j}=(-1)^{j-l+1} \sum_{t \neq j, l} \frac{\partial m_{q t}}{\partial x_{\gamma}} \operatorname{cof}^{l}\left(m_{k j}\right)
$$

vi) $c_{q v}=\frac{\partial m_{q v}}{\partial x_{\gamma}} \operatorname{cof}^{j}\left(m_{k l}\right)$ para $v \neq l, j$ e $q \neq k$. 
Quarto Passo: Consideremos as matrizes

$$
D_{q}=\sum_{i \neq i, j} \frac{\partial m_{q i}}{\partial x_{\gamma}}\left(\sum_{u \neq k}(-1)^{\gamma} R_{q u} \operatorname{cof}_{u i}^{j}\left(m_{k l}\right)\right)
$$

onde $q \neq k$ e

$$
\gamma=\left\{\begin{array}{l}
u+i-1, \text { se } j<i \text { e } u<k \text { ou } j>i \text { e } u>k \\
u+i, \text { se } j<i \text { e } u>k \text { ou } j>i \text { e } u<k .
\end{array}\right.
$$

Vamos olhar para cada entrada da matriz $D$ :

i) $d_{q l}=\sum_{i \neq i, j} \frac{\partial m_{q i}}{\partial x_{\gamma}}\left(\sum_{u \neq k}(-1)^{\gamma} m_{u l} \operatorname{cof}_{u i}^{j}\left(m_{k l}\right)\right)$.

Notemos que,

$$
\begin{aligned}
\sum_{u \neq k}(-1)^{\gamma} m_{u l} \operatorname{cof}_{u i}^{j}\left(m_{k l}\right)= & (-1)^{k+i} \sum_{l<i<j}(-1)^{u+i} m_{u l} \operatorname{det}\left(M_{k i, u l}^{j}\right)+\sum_{i<l<j}(-1)^{u+i-1} m_{u l} \operatorname{det}\left(M_{k i, u l}^{j}\right) \\
& +\sum_{l<j<i}(-1)^{u+i} m_{u l} \operatorname{det}\left(M_{k i, u l}^{j}\right)=(-1)^{k+i} \sum_{u \neq k}(-1)^{\sigma} m_{u l} \operatorname{det}\left(M_{k i, u l}^{j}\right),
\end{aligned}
$$

onde $\sigma$ é o valor de $u+i$ na matriz $M_{k i, u l}^{j}$.

Logo,

$$
d_{q l}=\sum_{i \neq i, j} \frac{\partial m_{q i}}{\partial x_{\gamma}}\left((-1)^{\beta} \sum_{u \neq k}(-1)^{\sigma} m_{u l} \operatorname{det}\left(M_{k i, u l}^{j}\right)\right)=\sum_{i \neq i, j} \frac{\partial m_{q i}}{\partial x_{\gamma}}(-1)^{\beta} \operatorname{det}\left(M_{k i}^{j}\right),
$$

onde

$$
\beta=\left\{\begin{array}{l}
k+i, \text { se } j>i \\
k+i-1, \text { se } j<i u<k
\end{array}\right.
$$

Assim,

$$
d_{q l}=\sum_{i \neq j, l} \frac{\partial m_{q i}}{\partial x_{\gamma}} \operatorname{cof}^{j}\left(m_{k i}\right)
$$

ii) $\quad d_{q j}=\sum_{i \neq i, j} \frac{\partial m_{q i}}{\partial x_{\gamma}}\left(\sum_{u \neq k}(-1)^{\gamma} m_{u j} \operatorname{cof}_{u i}^{j}\left(m_{k l}\right)\right)$. Procedendo de forma análoga ao feito no item anterior, obtemos temos que

$$
d_{q j}=(-1)^{l-j-1} \sum_{i \neq i, j} \frac{\partial m_{q i}}{\partial x_{\gamma}} \operatorname{cof}^{l}\left(m_{k j}\right)
$$

iii) Para $t \neq j, l$, temos

$$
d_{q t}=\sum_{i \neq i, j} \frac{\partial m_{q i}}{\partial x_{\gamma}}\left(\sum_{u \neq k}(-1)^{\gamma} m_{u t} \operatorname{cof}_{u i}^{j}\left(m_{k l}\right)\right)
$$


Notemos que para $i \neq t$, temos

$$
\sum_{u \neq k}(-1)^{\gamma} m_{u t} \operatorname{cof}_{u i}^{j}\left(m_{k l}\right)=0
$$

pois esta é a expressão do determinante de uma matriz que que tem duas colunas iguais a t-ésima coluna de $M$.

Logo,

$$
d_{q t}=\frac{\partial m_{q t}}{\partial x_{\gamma}}\left(\sum_{u \neq k}(-1)^{\gamma} m_{u t} \operatorname{cof}_{u t}^{j}\left(m_{k l}\right)\right) .
$$

Suponhamos, sem perda de generalidade que $t<j$. Então,

$$
\begin{aligned}
\sum_{u \neq k}(-1)^{\gamma} m_{u t} \operatorname{cof}_{u t}^{j}\left(m_{k l}\right) & =\sum_{u \neq k}(-1)^{u+t}(-1)^{k+l-1} m_{u t} \operatorname{det}\left(M_{k l, u t}^{j}\right) \\
& =(-1)^{k+l} \sum_{u \neq k}(-1)^{u+t-1} m_{u t} \operatorname{det}\left(M_{k l, u t}^{j}\right)=-\operatorname{cof}^{j}\left(m_{k l}\right)
\end{aligned}
$$

Portanto,

$$
d_{q t}=-\frac{\partial m_{q t}}{\partial x_{\gamma}} \operatorname{cof}^{j}\left(m_{k l}\right)
$$

i) $d_{s v}=0$, para $s \neq q$;

Quinto Passo: Para obter o resultado da proposição, basta considerar a matriz $E=\left(e_{q v}\right)$ dada por $E=C+\sum_{q \neq i} D i$.

Proposição 2.6.4. As matrizes $\Delta_{(\gamma, \nu)}^{(j, t)} E_{k l}$ pertencem ao $\mathcal{G}$-espaço tangente da matriz $M$, para $1 \leq k \leq n$ e $1 \leq j, t \leq n+1$.

\section{Demonstração.}

Vamos mostrar que $\Delta_{(\gamma, \nu)}^{(j, t)} E_{k l}$ são obtidas usando as matrizes da proposição 2.6.3. Para simplificar a notação, vamos omitir os índices ${ }_{(\gamma, \nu)} E_{k l}$ de $\Delta_{(\gamma, \nu)}^{(j, t)} E_{k l}$ quando o contexto for claro.

Vamos dividir a demonstração em dois casos:

i) Se $l=t$ e $i \neq t$, então

$$
\frac{\partial f_{j}}{\partial x_{\gamma}}\left(\frac{\partial f_{t}}{\partial x_{\nu}} E_{k t}+(-1)^{t-i+1} \frac{\partial f_{t}}{\partial x_{\nu}} E_{k j}\right)-\frac{\partial f_{j}}{\partial x_{\nu}}\left(\frac{\partial f_{t}}{\partial x_{\gamma}} E_{k t}+(-1)^{t-i+1} \frac{\partial f_{t}}{\partial x_{\gamma}} E_{k i}\right)=\Delta^{(j, t)} E_{k t}
$$


ii) Se $l \neq t$ e $i \neq l$. Consideremos as matrizes

$$
A=\frac{\partial f_{j}}{\partial x_{\gamma}}\left(\frac{\partial f_{t}}{\partial x_{\nu}} E_{k l}+(-1)^{l-t+1} \frac{\partial f_{l}}{\partial x_{\nu}} E_{k t}\right)-\frac{\partial f_{j}}{\partial x_{\nu}}\left(\frac{\partial f_{t}}{\partial x_{\gamma}} E_{k l}+(-1)^{l-t+1} \frac{\partial f_{l}}{\partial x_{\gamma}} E_{k t}\right)
$$

e

$$
B=\frac{\partial f_{j}}{\partial x_{\gamma}}\left(\frac{\partial f_{l}}{\partial x_{\nu}} E_{k t}+(-1)^{t-l+1} \frac{\partial f_{t}}{\partial x_{\nu}} E_{k l}\right)-\frac{\partial f_{j}}{\partial x_{\nu}}\left(\frac{\partial f_{l}}{\partial x_{\gamma}} E_{k t}+(-1)^{t-l+1} \frac{\partial f_{t}}{\partial x_{\gamma}} E_{k l}\right) .
$$

Notemos que

$$
\begin{aligned}
& A=\Delta^{(j, t)} E_{k l}+(-1)^{l-t+1} \Delta^{(j, l)} E_{k t} \in T \mathcal{G} M \\
& B=(-1)^{t-l+1} \Delta^{(j, t)} E_{k l}+\Delta^{(j, l)} E_{k t} \in T \mathcal{G} M .
\end{aligned}
$$

Então,

$$
A-(-1)^{l-t+1} B=\left(\Delta^{(j, t)}+\Delta^{(j, t)}\right) E_{k l}+(-1)^{l-t+1}\left(\Delta^{(j, l)}-\Delta^{(j, l)}\right) E_{k t}=\Delta^{(j, t)} E_{k l} \in T \mathcal{G} M .
$$

Proposição 2.6.5. As matrizes $\Delta_{i}^{j} E_{k l}$ pertencem ao $\mathcal{G}$-espaço tangente da matriz $M$ para todo $j \in V$ e $i \in L$, onde $\Delta_{i}^{j}$ denota menores maximais da matriz jacobiana de $f$ como definimos acima.

\section{Demonstração.}

Seja $d$ o posto maximal de $J M(X)$. Vamos provar o resultado usando indução sobre $d$. O caso $d=2$ é exatamente a proposição anterior.

Suponhamos que se posto $(J M(X))=d-1$, então $\Delta_{i}^{j} E_{k l}$ pertencem ao $\mathcal{G}$ - espaço tangente da matriz $M$ para todo $j \in V$ e $i \in L$.

Vamos provar o resultado para o caso em que o posto maximal de $J M(X)$ é $d$. Notemos que para cada $i \in L$ e $j \in V$, temos

$$
\Delta_{i}^{j}=\sum_{\substack{\alpha \notin i \\ \nu \notin j}} \frac{\partial f_{\alpha}}{\partial x_{\nu}} \operatorname{cof}\left(\frac{\partial f_{\alpha}}{\partial x_{\nu}}\right)
$$

Como cof $\left(\frac{\partial f_{\alpha}}{\partial x_{\nu}}\right)$ é dado em função do determinante de uma matriz $(d-1) \times(d-1)$, segue da hipótese de indução que

$$
\operatorname{cof}\left(\frac{\partial f_{\alpha}}{\partial x_{\nu}}\right) E_{k l} \in T \mathcal{G} M
$$

para todo $k, l$.

O resultado segue, pois $T \mathcal{G} M$ é um módulo sobre $\mathcal{P}$. 
Teorema 2.6.1. Sejam $M$ um germe de matriz $n \times(n+1)$ com entradas no ideal maximal de $\mathcal{O}_{r}$, definindo uma singularidade isolada Cohen-Macaulay de codimensão dois e $X=(f \circ M)^{-1}(0)$. Então, as seguintes condições são equivalentes:

(a) Existe um inteiro positivo $k$ tal que $\mathfrak{M}^{k} M a t_{(n, n+1)}(\mathcal{P}) \subset T \mathcal{G} M$;

(b) $M$ é $\mathcal{G}$-finitamente determinada;

(c) $M$ é transversal aos estratos da estratificação de $\operatorname{Mat}_{(n, n+1)}(\mathbb{C})$ fora da origem;

(d) $X \cap V\left(J_{f}\right)=\{0\}$;

(e) $I_{\mathcal{G}} M=J_{f}+<f_{1}, \ldots, f_{n+1}>\supseteq \mathfrak{M}^{k}$, para algum inteiro positivo $k$.

\section{Demonstração.}

Notemos que as implicações $(a) \Longrightarrow(b) \Longleftrightarrow(c)$, já foram demonstradas anteriormente. $(c) \Longrightarrow(d)$

Seja $\Delta$, como anteriormente a subvariedade irredutível de $\operatorname{Mat}_{(n, n+1)}(\mathbb{C})$ formada pelas matrizes singulares. Se $M$ é transversal à órbita da estratificação de $\operatorname{Mat}_{(n, n+1)}(\mathbb{C})$ fora da origem e, como por hipótese, $M$ define uma variedade com singularidade isolada na origem segue que $M \cap \Delta=\emptyset$ em $U-\{0\}$, onde $U$ é uma vizinhança da origem em $\mathbb{C}^{r}$. Isto é, $X \cap \mathcal{V}\left(J_{f}\right)=\{0\}$.

Usando o Teorema dos zeros de Hilbert (Nullstellensatz) no anel $\mathcal{O}_{r}$, (ver [20]), é imediato que $(d) \Longrightarrow(e)$.

Para mostrar, finalmente, que $(e) \Longrightarrow(a)$, usamos a Proposição 2.6 .1 para mostrar que $\left\langle f_{1}, \ldots, f_{n+1}\right\rangle E_{i j} \in T \mathcal{G} M$ e, a Proposição 2.6.4, para mostrar que $J_{f} E_{i j} \in T \mathcal{G} M$. Logo, se $I_{\mathcal{G}} M \supseteq \mathfrak{M}^{k}$, então existe $k_{1} \in \mathbb{N}$ tal que $\mathfrak{M}^{k_{1}} M a t_{(n, n+1)}(\mathcal{P}) \subseteq T_{\mathcal{G}} M$. 


\section{Capítulo 3}

\section{$\mathcal{G}$ - Equivalência Topológica de Matrizes}

Como aplicação dos resultados obtidos na seção anterior vamos estudar neste capítulo a $\mathcal{G}$ trivialidade topológica de família de matrizes. Os principais resultados são os de trivialidade topológica de deformações de germes quase- homogêneos de matrizes.

Concentraremos nosso estudo em matrizes $M, n \times n+1$, com entradas no ideal maximal de $\mathbb{K}\left\{x_{1}, \ldots, x_{r}\right\}$, onde $\mathbb{K}=\mathbb{R}$ ou $\mathbb{C}$. Denotamos por $f=\left(f_{1}, \ldots, f_{n+1}\right)$ o ideal gerado por seus menores maximais, $X$ a variedade definida por $f$ e por $\mathcal{P}^{0}$ o anel dos germes de funções contínuas de $\mathbb{K}^{r} \longrightarrow \mathbb{K}$.

\subsection{Um Teorema de Trivialidade Topológica}

Definição 3.1.1. Dizemos que dois germes de matrizes $M, N:\left(\mathbb{K}^{r}, 0\right) \longrightarrow M_{(n, n+1)}(\mathcal{P})$ são topologicamente equivalentes (ou $C^{0}-\mathcal{G}$-equivalentes), se existem um germe de homeomorfismo $\phi:\left(\mathbb{K}^{r}, 0\right) \longrightarrow\left(\mathbb{K}^{r}, 0\right)$ e matrizes invertiveis $A \in G L_{n}\left(\mathcal{P}^{0}\right)$ e $B \in G L_{n+1}\left(\mathcal{P}^{0}\right)$ tais que $M=A(N \circ \phi) B^{-1}$.

Notemos que esta é uma versão topológica da $\mathcal{G}$-equivalência definida anteriormente.

Uma função controle $\rho: \mathbb{K}^{r} \longrightarrow \mathbb{K}$ é uma função não negativa que satisfaz à seguinte condição:

i) $\rho(0)=0$ e existem constantes $c>0$ e $\alpha>0$ tais que $\rho(x) \geq c|x|^{\alpha}$ (isto é, $\rho$ satisfaz à condição de Lojasiewicz).

Observemos que a condição (i) implica que 0 é um zero isolado da função $\rho$. No caso analítico, a recíproca também se verifica (Ver [52], pág. 518). 
Definição 3.1.2. Um germe de matriz $M \in \operatorname{Mat}_{(n, n+1)}(\mathcal{P})$ é $k-C^{0}-\mathcal{G}$-determinado, se para toda matriz $N$ tal que $j^{k} M(0)=j^{k} N(0), N$ é $C^{0}-\mathcal{G}$-equivalente a $M$.

Definição 3.1.3. Uma deformação a um parâmetro $M:\left(\mathbb{K}^{r} \times \mathbb{K}, 0\right) \longrightarrow \operatorname{Mat}_{(n, n+1)}(\mathcal{P})$ de $M_{0}:\left(\mathbb{K}^{r}, 0\right) \longrightarrow \operatorname{Mat}_{(n, n+1)}(\mathcal{P})$ é $C^{0}-\mathcal{G}$-trivial se satisfaz às seguintes condições:

a) Existe um homeomorfismo

$$
\begin{aligned}
\Phi:\left(\mathbb{K}^{r} \times \mathbb{K}, 0\right) & \longrightarrow\left(\mathbb{K}^{r} \times \mathbb{K}, 0\right) \\
(x, t) & \longmapsto(\phi(x, t), t)
\end{aligned}
$$

tal que $\Phi(x, 0)=(x, 0)$;

b) Existem familias de matrizes $A \in G L_{n}\left(\mathcal{P}^{0}\right)$ e $B \in G L_{n+1}\left(\mathcal{P}^{0}\right)$ tais que $A(x, 0)=I d_{n+1}$, $B(x, 0)=I d_{n}$ e $M_{0}=A(M \circ \phi) B$.

Proposição 3.1.1. Sejam $M_{0}$ um germe de matriz $n \times n+1$, definindo uma singularidade Cohen-Macaulay de codimensão 2 e $M$ uma deformação de $M_{0}$. Suponha que existe uma função de controle $\rho$ tal que

$$
\rho(x, t)^{2} \frac{\partial M}{\partial t}=\sum_{i=1}^{r} \xi_{i}(x, t) \frac{\partial M}{\partial t}+L M+M R,
$$

$\operatorname{com}_{i}(x, t) \in \mathbb{K}\{x, t\}, L \in \operatorname{Mat}_{(n, n)}(\mathbb{K}\{x, t\})$ e $R \in \operatorname{Mat}_{(n+1, n+1)}(\mathbb{K}\{x, t\})$ tais que

$$
\frac{\left|\xi_{i}(x, t)\right|}{\rho(x, t)^{2}} \leq C_{1}|x|, \quad \frac{\left|L_{i j}(x, t)\right|}{\rho(x, t)^{2}} \leq C_{2}|x|, \quad e \frac{\left|R_{i j}(x, t)\right|}{\rho(x, t)^{2}} \leq C_{3}|x| .
$$

Então, a família $M$ é $C^{0}-\mathcal{G}$-trivial.

\section{Demonstração.}

A demonstração é análoga ao que foi feito para o Teorema de Determinação Finita. A diferença é que aqui, vamos construir campos de vetores contínuos e a condição (3.1) garante a unicidade do fluxo correspondente.

Seja $V(x, t)$, o campo de vetores em $\mathbb{K}^{r} \times \mathbb{K}, 0$ definido por

$$
\left\{\begin{array}{l}
\frac{\partial}{\partial t}-\sum_{i=1}^{K} \frac{\xi_{i}(x, t)}{\rho^{2}(x, t)} \frac{\partial}{\partial x_{i}}, \text { se } x \neq 0 \\
\frac{\partial}{\partial t}, \text { se } x=0
\end{array}\right.
$$

Para cada $j, V_{j}$ denota a $j$-ésima componente de $V$. O campo $V$ é real analítico ao longo de $\left(\mathbb{K}^{r} \times \mathbb{K}\right) \backslash(\{0\} \times \mathbb{K})$. Além disso,

$$
\left|V_{j}(x, t)\right|=\frac{\left|\xi_{i}(x, t)\right|}{\rho^{2}(x, t)} \leq C|x|,
$$


para $1 \leq j \leq r$, isto é, $V_{j}(x, t)$ satisfaz uma condição do tipo Lipschitz ao longo de $0 \times \mathbb{K}$. Segue de [33] que o campo $V$ é localmente integrável. Para maiores detalhes no caso complexo, ver [40]. Vamos indicar por $\Phi(x, t)$ o fluxo correspondente.

Para construir matrizes $A$ e $B$ como acima, podemos usar argumentos análogos aos que foram usados no Lema (2.3.1), isto é, precisamos encontrar matrizes $N=N(x, t) \in G L_{n}(\mathbb{K}\{x, t\})$ e $S=S(x, t) \in G L_{p}(\mathbb{K}\{x, t\})$ tais que

$$
\begin{aligned}
& N(x, 0)=I d_{n}, \\
& S(x, 0)=I d_{n+1}
\end{aligned}
$$

e satisfaçam o seguinte sistema de equações diferenciais

$$
\rho^{2} \frac{\left(\partial N M_{0} S\right)_{i j}}{\partial t}=-\left(L N M_{0} S+N M_{0} S R\right)_{i j}, \forall 1 \leq i \leq k, 1 \leq j \leq p .
$$

Neste caso, basta resolver os dois seguintes sistemas de equações diferenciais

$$
\begin{aligned}
& \rho^{2} \frac{\partial N_{i j}}{\partial t}=-\left(L(N)_{i j}, \forall 1 \leq i \leq n+1,1 \leq j \leq n\right. \\
& \rho^{2} \frac{\partial S_{i j}}{\partial t}=-(S R)_{i j}, \forall 1 \leq i \leq n+1,1 \leq j \leq n .
\end{aligned}
$$

com as mesmas condições iniciais e já resolvemos o sistema original.

Considere o seguinte campo de vetores $W=\left(w_{i j}\right)$ em $\mathbb{K}^{(n+1)(n+1)} \times \mathbb{K}$

$$
W(x, y, t)=\frac{\partial}{\partial t}+\sum_{k=1}^{n+1} \frac{L_{i k}}{\rho^{2}} y_{j k} E_{i j}
$$

Em (3.2), usamos $y_{j k}$ para denotar as variáveis de $\operatorname{Mat}_{(n, n+1)}(\mathbb{C})$, onde $1 \leq j \leq n$ e $1 \leq k \leq$ $n+1$. Por hipótese,

$$
\frac{L_{i j}(x, t)}{\rho(x, t)^{2}} \leq C_{2}|x|,
$$

segue de [40] que o campo é integrável.

De maneira análoga, acontece se consideramos o campo de vetores em $\mathbb{K}^{n^{2}} \times \mathbb{K}$

$$
Z(x, y, t)=\frac{\partial}{\partial t}+\sum_{l=1}^{n} \frac{S_{j l}}{\rho^{2}(\phi(x), t)} y_{i l} .
$$




\subsection{Deformações de Germes Quase- Homogêneneos}

Nosso próximo objetivo é demonstrar o Teorema da Trivialidade Topológica para deformações de germes quase-homogêneos. O interesse é mostrar que para deformações de grau maior ou igual ao máximo dos graus de quase- homogeneidade das entradas da matriz, a hipótese da Proposição (3.1.1) se verifica.

Definição 3.2.1. Dados $\left(a_{1}, \ldots, a_{r}\right) \in \mathbb{N}^{r}$, para qualquer monômio $x^{\alpha}=x_{1}^{\alpha_{1}} x_{2}^{\alpha_{2}} \ldots x_{r}^{\alpha_{r}}$, definimos

$$
f i l\left(x^{\alpha}\right)=\sum_{i=1}^{r} a_{i} \alpha_{i} .
$$

Podemos definir uma filtração no anel $\mathcal{P}$, via a filtração definida anteriormente da seguinte maneira

$$
f i l(f)=\inf _{\alpha}\left\{f i l\left(x^{\alpha}\right) \mid \frac{\partial^{\alpha} f}{\partial x^{\alpha}}(0) \neq 0\right\},
$$

para qualquer germe $f \in \mathcal{P}$. Podemos estender esta definição para o anel de famílias a $s$ parâmetros de germes em $r$ variáveis, colocando

$$
f i l\left(x^{\alpha} t^{\beta}\right)=f i l\left(x^{\alpha}\right)
$$

Dada uma matriz $M \in \operatorname{Mat}_{(n+1, n)}(\mathcal{P}), M=\left(m_{i j}\right)$, definimos $f i l(M)=D=\left(d_{i j}\right)$, onde $d_{i j}=f i l\left(m_{i j}\right)$ para $1 \leq i \leq n, 1 \leq j \leq n+1$.

Definição 3.2.2. Dizemos que um germe de matriz $M \in \operatorname{Mat}_{(n, n+1)}(\mathcal{P})$ é quase- homogêneo do tipo $(D ; a) \in \operatorname{Mat}_{(n, n+1)}(\mathbb{N}) \times \mathbb{N}^{r}$, se

i) $\operatorname{fil}\left(m_{i j}\right)=d_{i j}$ com relação $\grave{a} \quad a=\left(a_{1}, \ldots, a_{r}\right)$;

ii) As seguintes relações são verificadas

$$
d_{i j}-d_{i k}=d_{l j}-d_{l k} \text { para todo } 1 \leq i, l \leq n, 1 \leq j, k \leq n+1
$$

Seja $M$ uma matriz $n \times(n+1)$ quase-homogênea de tipo $(D ; a)$. Seja $f_{u}$ um menor maximal de $M$, obtido eliminando a coluna $u \operatorname{com} u=1, \ldots, n+1$. Então, é imediato que $f_{u}$ é quasehomogêneo de tipo $\left(a ; D_{1}, \ldots, D_{n}\right)$, onde $f i l\left(f_{u}\right)=D_{u}=\sum_{j \neq u} d_{i j}$. Em [16], encontramos uma demonstração que a recíproca também é verdadeira, isto é, se $f \subset \mathbb{C}\left\{x_{1}, \cdots, x_{r}\right\}$ é um ideal Cohen-Macaulay de codimensão 2 gerado por polinômios quase-homogêneos com relação à algum peso $a$, então é possível encontrar uma matriz de representação $M$ de $f$ que é quasehomogênea do tipo $(D, a)$ para algum $D \in \operatorname{Mat}_{(n, n+1)}(\mathbb{N})$. 
Seja $k_{1}=m . m . c\left\{D_{u} \mid 1 \leq u \leq n+1\right\}$ e $\beta_{u}=k_{1} / D_{u}$. Definimos

$$
\mathcal{N}_{\mathcal{H}} M=\sum_{j=1}^{n} f_{j}^{2 \beta_{j}}
$$

Observemos que esta é uma função quase-homogênea de tipo $\left(2 k_{1} ; a\right)$.

Além disso, cada $\frac{\partial f_{j}}{\partial x_{s}}$ é quase-homogênea de tipo $\left(a, D_{j}-a_{s}\right)$ e para cada menor da matriz jacobiana de $f$, existe um inteiro $d_{i}, i \in I$, tal que $\Delta_{i}$ é quase-homogêneo de tipo $\left(d_{i} ; a\right)$. Seja $k_{2}=m . m . c .\left(d_{i}\right)$ e $\alpha_{i}=k_{2} / d_{i}$. Definimos

$$
\mathcal{N}_{\mathcal{R}} M=\sum_{i \in I} \Delta_{i}^{2 \alpha_{i}}
$$

Logo, esta é uma função quase-homogênea de tipo $\left(2 k_{2} ; a\right)$.

Seja $K=m . m . c\left\{k_{1}, k_{2}\right\}$ e $\alpha_{i}=K / k_{i}$. Definimos

$$
\mathcal{N}_{\mathcal{G}} M=\mathcal{N}_{\mathcal{R}}^{\alpha_{1}} M+\mathcal{N}_{\mathcal{H}}^{\alpha_{2}} M
$$

Dessa maneira, $\mathcal{N}_{\mathcal{G}} M$ é quase- homogênea de tipo $(a ; 2 K)$.

Seja $M_{t}$ uma deformação de $M$. Definimos a função $\mathcal{N}_{\mathcal{R}} M_{t}=\sum_{i \in I}\left(\Delta_{t}\right)_{i}^{2 \alpha_{i}}$, onde $\left(\Delta_{t}\right)_{i}$ são os menores $d \times d$ da matriz jacobiana de $f_{t}$ e os $\alpha_{i}$ são os mesmos definidos acima. Se $M_{t}$ é quase-homogênea de mesmo tipo que $M$, então $\mathcal{N}_{\mathcal{R}} M_{t}$ é quase-homogênea de tipo $2 k_{2}$.

Podemos definir de maneira análoga, o controle $\mathcal{N}_{\mathcal{H}} M_{t}=\sum_{i=1}^{n+1} f_{t i}^{2 \beta_{i}}$, onde cada $\beta_{i}$ é obtido como acima e $\left(f_{t}\right)_{i}$ são os menores $n \times n$ da matriz $M_{t}$.

Vamos demonstrar o Teorema da Trivialidade Topológica em duas partes. Primeiro, mostramos o resultado para o caso em que estamos trabalhando apenas com o grupo $\mathcal{H}$ e $\mathcal{N}_{\mathcal{H}}$ é uma função de controle. Em seguida, generalizamos a demonstração para o caso do grupo $\mathcal{G}$ com a condição que $\mathcal{N}_{\mathcal{G}}$ é uma função de controle.

Proposição 3.2.1. Seja $M_{0}:\left(\mathbb{K}^{r}, 0\right) \longrightarrow \operatorname{Mat}_{(n, n+1)}(\mathcal{P})$ um germe de matriz quase-homogêneo de tipo $(D, a) \in \operatorname{Mat}_{(n, n+1)}(\mathbb{N}) \times \mathbb{N}^{r}$, satisfazendo à condição $\mathcal{N}_{\mathcal{H}}\left(M_{0}(x)\right) \geq c|x|^{\alpha}$ para constantes c e $\alpha$. Então,

i) Deformações $M(x, t)=M_{0}(x)+t \Theta(x)$ de $M_{0} \operatorname{com} f i l\left(\Theta_{i j}\right) \geq d_{\max }+1, \forall i, j, t \in[0,1]$, e $d_{\text {max }}=\max _{i j}\left\{d_{i j}\right\}$, são $\mathcal{C}^{0}-\mathcal{H}$-triviais.

ii) Deformações $M(x, t)=M_{0}(x)+t \Theta(x)$ de $M_{0} \operatorname{com} f i l\left(\Theta_{i j}\right) \geq d_{\max }, \forall i, j$, são $\mathcal{C}^{0}-$ $\mathcal{H}$-triviais para t suficientemente pequeno. 


\section{Demonstração.}

Mostremos inicialmente o item $i)$. Seja $M(x, t)=M_{0}(x)+t \Theta(x)$ uma deformação de $M_{0}$ $\operatorname{com} \operatorname{fil}\left(\Theta_{i j}\right) \geq d_{\max }+1, \forall i, j, t \in[0,1]$. Obtemos a $\mathcal{C}^{0}-\mathcal{H}$-trivialidade da família construindo germes de aplicações $\xi_{i}, \delta_{j}$ de classe $\mathcal{C}^{0}, \xi_{i}, \delta_{j}: \mathbb{K}^{r} \times \mathbb{K} \longrightarrow \mathbb{K}$ tais que

$$
\left(\frac{\partial M}{\partial t}\right)_{i j} E_{i j}=\sum_{l=1}^{n+1} \sum_{r=1}^{n+1} \xi(x, t) C_{r l}(M)+\sum_{l=1}^{n} \sum_{i=1}^{n} \delta(x, t) R_{i l}(M),
$$

onde $C_{j l}\left(R_{j l}\right)$ é a matriz que tem na $j$-ésima coluna (linha) a $l$-ésima coluna (linha) de $M$ e zero em qualquer outro lugar.

Uma vez que, usando a Proposição 2.6.1, podemos escrever

$$
f_{k}\left(\frac{\partial M}{\partial t}\right) E_{i j}=\left(\frac{\partial M}{\partial t}\right) \sum_{i j}^{n+1}(-1)^{i+l} M_{k ; l i} C_{j l}
$$

então, multiplicando por $f_{k}^{2 \alpha_{i}-1}$ e somando em $k$, obtemos

$$
\left(\frac{\partial M}{\partial t}\right) \mathcal{N}_{\mathcal{H}} M E_{i j}=\sum_{k=1}^{n+1}\left(\frac{\partial M}{\partial t}\right)_{i j}\left(\sum_{l=1}^{n+1}(-1)^{i+l} f_{k}^{2 \alpha_{k}-1} M_{k ; l i}\right) C_{j l}(M),
$$

definimos:

$$
Q_{l k}(x, t)=(-1)^{i+l} f_{t k}^{2 \alpha_{k}-1} M_{k ; l i}\left(\frac{\partial M}{\partial t}\right)_{i j}
$$

Então,

$$
\left(\frac{\partial M}{\partial t}\right) E_{i j}=M\left(\sum_{l=1}^{n+1} \sum_{k=1}^{n+1} \frac{Q_{l k}(x, t)}{\mathcal{N}_{\mathcal{H}} M} E_{l j}\right)
$$

Agora,

i) $f i l\left(M_{k ; i}\right) \geq d_{z s}+1$, com $z \neq i, s \neq l, k$.

ii) $f i l\left(f_{t k}^{2 \alpha_{k}-1}\right) \geq 2 k_{1}-d_{k}$, onde $d_{k}=d_{z s}+d_{b c}$;

iii) $f i l\left(Q_{l k}\right) \geq 2 k_{1}-d_{k}+d_{t s}+d_{\max } \geq 2 k_{1}+1$, para todo $k$, com $d_{\max }=\max _{i j}\left\{d_{i j}\right\}$.

Então, para cada $l, k, \frac{Q_{l k}(x, t)}{\mathcal{N}_{\mathcal{H}} M_{t}} \leq C|x|$ e, portanto, como na Proposição (3.1.1) o campo

$$
W(x, y, t)=\frac{\partial}{\partial t}+\sum_{k=1}^{n+1} \frac{Q_{l k}}{\mathcal{N}_{\mathcal{H}} M_{t}} y_{j k} E_{i j} .
$$

é integrável, o que implica a $\mathcal{C}^{0}-\mathcal{H}$-trivialidade de $M$.

Para demonstrar o item $i i)$ seja $M(x, t)=M_{0}(x)+t \Theta(x)$ uma deformação de $M_{0}$ com $f i l\left(\Theta_{i j}\right) \geq d_{\max }, \forall i, j$ e $t$ suficientemente pequeno. Analogamente ao feito no item $\left.i\right)$, conside- 
ramos o campo de vetores $p(x, y, t) W(x, y, t)$, onde $W(x, y, t)$ é o campo definido na Proposição (3.1.1) e $p: \mathbb{K}^{r} \times \operatorname{Mat}_{(n, n+1)}(\mathcal{P}) \times \mathbb{K} \longrightarrow \mathbb{K}$ é uma função bacia cônica, ( ver [38], Lema 4), tal que $\left.p\right|_{\mathbb{K}^{r} \times \operatorname{Mat}_{(n+1), n}(\mathcal{P}) \times \mathbb{K}-\{0,0, t\}}$ é suave e satisfaz

$$
\left\{\begin{array}{l}
p(x, y, t)=1, \text { para todo }(x, y, t) \in \bar{U} \\
p(x, y, t)=0 \text { fora de } V \\
0 \leq p(x, y, t) \leq 1 \text { em } V-\bar{U} \\
p(0,0, t)=0, \text { para todo } t
\end{array}\right.
$$

$V$ e $U$ são vizinhanças da região $|Y|<c \rho(x)^{1 / 2}$ em $\mathbb{K}^{r} \times \operatorname{Mat}_{(n, n+1)}(\mathcal{P}) \times \mathbb{K}-\{0,0, t\}$,

$$
V=\left\{(x, Y, t) \text { tal que }|Y| \leq c_{1} \rho(x)^{1 / 2}\right\}
$$

e $U$ é escolhido tal que $U \subset \bar{U} \subset V$. Então,

$$
\left|p(x, y, t) W_{i j}(x, y, t)\right|=\left|\frac{L_{i j}(x, t)}{\mathcal{N}_{\mathcal{H}} M_{t}}\right||p y| \leq\left|\frac{L_{i j}(x, t)}{\left(\mathcal{N}_{\mathcal{H}} M_{t}\right)}\right| \mathcal{N}_{\mathcal{H}} M_{t}^{1 / 2}
$$

o que implica a integrabilidade do campo e, conseqüentemente a $\mathcal{C}^{0}-\mathcal{H}$ - trivialidade.

Teorema 3.2.1. Seja $M_{0}:\left(\mathbb{K}^{r}, 0\right) \longrightarrow \operatorname{Mat}_{(n, n+1)}(\mathcal{P})$ um germe de matriz quase-homogêneo de tipo $(D, a) \in \operatorname{Mat}_{(n, n+1)}(\mathbb{N}) \times \mathbb{N}^{r}$, satisfazendo a condição $\mathcal{N}_{\mathcal{G}}\left(M_{0}(x)\right) \geq c|x|^{\alpha}$ para constantes c e $\alpha$. Então, deformações $M(x, t)=M_{0}(x)+t \Theta(x), \operatorname{com} f i l\left(\Theta_{i j}\right) \geq d_{\max }, \forall i, j, t \in[0,1] e$ $d_{\text {max }}=\max _{i j}\left\{d_{i j}\right\}$, são $\mathcal{C}^{0}-\mathcal{G}$-triviais.

\section{Demonstração.}

Faremos a demonstração no caso em que $n=2$. O caso geral, é feito de maneira análoga. Provamos anteriormente que,

$$
\Delta_{I} E_{i p}=\left(\frac{\partial f_{p}}{\partial x_{r}}\left(\frac{\partial f_{q}}{\partial x_{s}} E_{i p}+\frac{\partial f_{p}}{\partial x_{s}} E_{i q}\right)-\frac{\partial f_{p}}{\partial x_{s}}\left(\frac{\partial f_{q}}{\partial x_{r}} E_{i p}+\frac{\partial f_{p}}{\partial x_{r}} E_{i q}\right)\right) .
$$

Como

$$
\frac{\partial f_{p}}{\partial x_{r}} E_{i q}+\frac{\partial f_{q}}{\partial x_{r}} E_{i p}=M_{p ; q i} \frac{\partial M}{\partial x_{r}}+\sum_{j=1}^{n+1} \frac{\partial M_{p ; j i}}{\partial x_{r}} C_{q j}+\sum_{j=1}^{n+1} \frac{\partial M_{q ; j i}}{\partial x_{r}} C_{p j}-\sum_{k, j, z} \frac{\partial m_{k j}}{\partial x_{r}} R_{k z}
$$

para $p \neq q, j \neq p, q$ e $z \neq i$, sejam

$$
\begin{aligned}
& \xi_{u}(x, t)=\frac{\partial f_{p}}{\partial x_{u}} M_{p ; q i}, \quad \varsigma_{u, v}(x, t)=\frac{\partial f_{p}}{\partial x_{u}} \frac{\partial M_{p ; j i}}{\partial x_{v}}, \\
& \delta_{u, v}(x, t)=\frac{\partial f_{p}}{\partial x_{u}} \frac{\partial M_{q ; j i}}{\partial x_{v}}, \quad Z_{u}(x, t)=\frac{\partial f_{p}}{\partial x_{u}} \frac{\partial m_{k j}}{\partial x_{s}},
\end{aligned}
$$


onde $u, v \in\{r, s\}, u \neq v$. Então,

$$
\Delta_{I} E_{i p}=\left(\sum_{u, v} \xi_{u} \frac{\partial M}{\partial x_{v}}-\left(\sum_{k, j, z} \sum_{u} Z_{u} E_{k z}\right) M+M\left(\sum_{j=1}^{n+1} \sum_{u, v} \delta_{u, v} E_{j p}+\sum_{j=1}^{n+1} \sum_{u, v} \varsigma_{u, v} E_{j q}\right)\right) .
$$

Sejam

$$
\begin{aligned}
L_{p} & =\sum_{j=1}^{n+1} \sum_{u, v} \delta_{u, v} E_{j p}+\sum_{j=1}^{n+1} \sum_{u, v} \varsigma_{u, v} E_{j q} \\
R_{p} & =\sum_{k, j, z} \sum_{u} Z_{u} E_{k z} .
\end{aligned}
$$

Logo,

$$
\Delta_{I} E_{i p}=\sum_{u, v} \xi_{u} \frac{\partial M}{\partial x_{v}}-R_{p} M+M L_{p}
$$

Portanto,

$$
\Delta_{I} E_{i p}\left(\frac{\partial M}{\partial t}\right)_{i p}=\left(\frac{\partial M}{\partial t}\right)_{i p}\left(\sum_{u, v} \xi_{u} \frac{\partial M}{\partial x_{v}}-R_{p} M+M L_{p}\right)
$$

Multiplicando a expressão anterior por $\Delta_{I}^{2 \alpha_{2}-1}$ e somando em $I$, temos

$$
\mathcal{N}_{\mathcal{R}} M\left(\frac{\partial M}{\partial t}\right)_{i p} E_{i p}=\sum_{I} \Delta_{I}^{2 \alpha_{2}-1}\left(\frac{\partial M}{\partial t}\right)_{i p}\left(\sum_{u, v} \xi_{u} \frac{\partial M}{\partial x_{v}}-R_{p} M+M L_{p}\right) .
$$

Usando a Proposição (3.2.1), temos

$$
\mathcal{N}_{\mathcal{H}} M\left(\frac{\partial M}{\partial t}\right)_{i p}=M\left(\sum_{l=1}^{n+1} \sum_{k=1}^{n+1} W_{l k}(x, t) E_{l p}\right) .
$$

Somando as duas últimas expressões, obtemos

$$
\mathcal{N}_{\mathcal{G}} M\left(\frac{\partial M}{\partial t}\right)_{i p} E_{i p}=\sum_{I} \Delta_{I}^{2 \alpha_{2}-1}\left(\frac{\partial M}{\partial t}\right)_{i p}\left(\sum_{u, v} \xi_{u} \frac{\partial M}{\partial x_{v}}-R_{p} M+M\left(L_{p}+\sum_{l=1}^{n+1} \sum_{k=1}^{n+1} W_{l k}(x, t) E_{l j}\right)\right)
$$

Seja $d_{\max }=\max _{i j}\left\{d_{i j}\right\}$ e observemos que,

i) $\operatorname{fil}\left(\frac{\partial f_{p}}{\partial x_{u}}\right) \geq d_{p}-a_{u}, \operatorname{com} j, k \neq p$;

ii) $f i l\left(\frac{\partial m_{k j}}{\partial x_{v}}\right) \geq d_{k j}-a_{v}$;

iii) $\quad$ fil $\left(\frac{\partial M}{\partial t}\right)_{i p} \geq d_{\text {max }}+1$, para todo $k$ 
iv) $\operatorname{fil}\left(M_{p ; q i}\right) \geq d_{z h}$, com $z \neq i$ e $h \neq p, q$;

v) $\operatorname{fil}\left(\Delta_{I}^{2 \alpha_{I}-1}\right) \geq 2 K-\left(d_{p}-a_{u}\right)-\left(d_{q}-a_{v}\right)$.

Observemos ainda que $d_{q}=d_{z h}+d_{k j}$, onde $k \neq z$. Então,

$$
\begin{aligned}
& f i l\left(\left(\frac{\partial M}{\partial t}\right)_{i p} \Delta_{I}^{2 \alpha_{I}-1} \xi_{u}(x, t)\right)=2 K-d_{q}+d_{k j}+d_{\max }+1 \geq 2 K+1 \\
& f i l\left(\left(\frac{\partial M}{\partial t}\right)_{i p} \Delta_{I}^{2 \alpha_{I}-1} \varsigma_{u, v}(x, t)\right) \geq 2 K+d_{\max }-d_{q}+d_{k j}+1 \geq 2 K+1 \\
& f i l\left(\left(\frac{\partial M}{\partial t}\right)_{i p} \Delta_{I}^{2 \alpha_{I}-1} Z_{u}(x, t)\right) \geq 2 K-d_{q}+d_{k j}+d_{\max }+1 \geq 2 K+1 \\
& f i l\left(\left(\frac{\partial M}{\partial t}\right)_{i p} \Delta_{I}^{2 \alpha_{I}-1} \delta_{u}(x, t)\right) \geq 2 K-d_{q}+d_{z h}+d_{\max }+1 \geq 2 K+1
\end{aligned}
$$

Para finalizar, basta observar que

$$
\begin{array}{llll}
\left(\frac{\partial M}{\partial t}\right)_{i p} \frac{\Delta_{I}^{2 \alpha_{I}-1} \xi_{u}(x, t)}{\mathcal{N}_{\mathcal{G}} M} \leq c_{1}|x|, & & \left(\frac{\partial M}{\partial t}\right)_{i p} \frac{\Delta_{I}^{2 \alpha_{I}-1} \varsigma_{u, v}(x, t)}{\mathcal{N}_{\mathcal{G}} M} \leq c_{2}|x| \\
\left(\frac{\partial M}{\partial t}\right)_{i p} \frac{\Delta_{I}^{2 \alpha_{I}-1} Z_{u}(x, t)}{\mathcal{N}_{\mathcal{G}} M} \leq c_{3}|x| \text { e } & & \left(\frac{\partial M}{\partial t}\right)_{i p} \frac{\Delta_{I}^{2 \alpha_{I}-1} \delta_{u}(x, t)}{\mathcal{N}_{\mathcal{G}} M} \leq c_{4}|x|
\end{array}
$$

e pela Proposição (3.1.1) segue a $\mathcal{C}^{0}-\mathcal{G}$-trivialidade.

Corolário 3.2.1. Seja $M_{0}:\left(\mathbb{K}^{r}, 0\right) \longrightarrow \operatorname{Mat}_{(n, n+1)}(\mathcal{P})$ um germe de matriz quase-homogêneo de tipo $(D, a) \in \operatorname{Mat}_{(n, n+1)}(\mathbb{N}) \times \mathbb{N}^{r}$, tal que a variedade definida por $M_{0}$ tem singularidade isolada. Então, deformações $M(x, t)=M_{0}(x)+t \Theta(x)$, com $f i l\left(\Theta_{i j}\right) \geq d_{\max }, \forall i, j$, e $d_{\max }=$ $\max _{i j}\left\{d_{i j}\right\}$, são $\mathcal{C}^{0}-\mathcal{G}$-triviais parat suficientemente pequeno.

\section{Demonstração.}

Se $\mathbb{K}=\mathbb{C}$, pelo Teorema 2.6.1 a hipótese que $X$ tem singularidade isolada implica que $M_{0}$ é $\mathcal{G}$-finitamente determinada. Então $\mathcal{N}_{\mathcal{G}}\left(M_{0}(x)\right)$ satisfaz a condição do Teorema anterior. Portanto, o resultado segue.

Quando $\mathbb{K}=\mathbb{R}$, como as entradas de $M_{0}$ são funções analíticas reais, então é possível mostrar (ver [52], Lema 6.2, pág. 518) que $\mathcal{G}\left(M_{0}\right)$ satisfaz uma condição de Lojasiewicz e, é portanto um controle. 
Exemplo 3.2.1. Seja $M_{0}=\left(\begin{array}{ccc}z & y & x \\ x & w & y^{3}+z^{3}\end{array}\right)$. Notamos que $M_{0}$ é $\mathcal{G}$-finitamente determinada, pois $\mathcal{M} M a t_{n, n+1}(\mathbb{C}) \subset T_{\mathcal{G}} M$. Portanto, pelo corolário anterior, todas as deformações $M$ de $M_{0}$ com filtração de grau maior que três são $\mathcal{C}^{0}-\mathcal{G}$-triviais. Além disso, deformações suficientemente pequenas de $M_{0}$ de grau exatamente 3 são $\mathcal{C}^{0}-\mathcal{G}$-triviais. 


\section{Capítulo 4}

\section{Deformações de germes}

\section{Cohen-Macaulay de Codimensão 2}

A teoria de deformações é o estudo infinitesimal de uma família na vizinhança de um determinado elemento. A definição de deformação envolve a noção de deformação plana. Intuitivamente, numa família plana, os membros dependem continuamente dos pontos do espaço de parâmetros. Neste capítulo definimos a noção de deformação de um germe analítico complexo $X_{0}$ e estudamos, em particular, deformações de germes Cohen-Macaulay de codimensão 2. As principais referências deste capítulo são [16] e [45].

\subsection{Deformações Infinitesimais}

Seja $\left(X_{0}, 0\right) \subset\left(\mathbb{C}^{r}, 0\right)$ um germe de variedade analítica, com anel local $\mathcal{O}_{X_{0}, 0}$. Como estamos considerando germes na origem, freqüentemente omitiremos o ponto 0 da nossa notação. Os primeiros termos da resolução de $\mathcal{O}_{X_{0}}$ são

$$
\mathcal{O}_{r}^{l} \stackrel{h}{\longrightarrow} \mathcal{O}_{r}^{k} \stackrel{f}{\longrightarrow} \mathcal{O}_{N} \longrightarrow \mathcal{O}_{X_{0}} \longrightarrow 0
$$

As entradas do vetor linha $f=\left(f_{1}, \ldots, f_{k}\right)$ geram o ideal $I$ de $X_{0}$ e as colunas de $h$ geram as relações.

Definição 4.1.1. Uma família de variedades analíticas com fibra especial $X_{0}$ sobre um espaço base $S$, contendo o ponto 0 é o germe de uma aplicação analítica $\pi:(X, 0) \longrightarrow(S, 0)$ tal que $X_{0}$ é isomorfo à $\pi^{-1}(0)$.

Em particular, no caso local, isto significa que existem equações $F_{1}(x, s), \ldots, F_{k}(x, s)$, cujas restrições a $s=0$ geram um ideal, isomorfo ao ideal de $X_{0}$. 
Seja $\pi:(X, 0) \longrightarrow(S, 0)$ uma aplicação entre germes analíticos complexos e suponha que seja dado um mergulho da fibra $\left(\pi^{-1}(0), 0\right) \subset\left(\mathbb{C}^{r}, 0\right)$. Então, $\pi$ pode ser realizada como a composição de um mergulho $(X, 0) \subset\left(S \times \mathbb{C}^{r}\right)$ e a projeção de $S \times \mathbb{C}^{r}$ no primeiro fator (ver [45]). Fixemos tal representação e denotemos por $\left(F_{1}, \ldots, F_{k}\right)$ o ideal $I$ de $(X, 0)$ em $\mathcal{O}_{S, 0} \widehat{\otimes} \mathbb{C}\left\{z_{1}, \ldots, z_{r}\right\}^{1}$. As aplicações $f_{i}=F_{i}(0, z)$ geram o ideal $I_{0}$ de $\left(X_{0}, 0\right)$ em $\mathbb{C}\left\{z_{1}, \ldots, z_{r}\right\}$.

Definição 4.1.2. A aplicação $\pi:(X, 0) \longrightarrow(S, 0)$ é plana em 0 se toda relação $\sum f_{i} r_{i}=0$ entre os $f_{i}^{\prime}$ s pode ser levantada para uma relação $\sum F_{i} R_{i}$ entre os $F_{i}$. Uma aplicação $\pi: X \longrightarrow$ $S$ é plana se ela é plana em todo ponto de $X$.

Proposição 4.1.1. ([45], pág. 8) A aplicação $\pi:(X, 0) \longrightarrow(S, 0)$ é plana em 0 se, e somente se, $\mathcal{O}_{X, 0}$ é um $\mathcal{O}_{S, 0}$ submódulo plano.

Definição 4.1.3. Uma deformação de um germe $\left(X_{0}, 0\right)$ é um germe de aplicação plana $\pi$ : $(X, 0) \longrightarrow(S, 0)$, tal que $\left(X_{0}, 0\right)$ é isomorfo à fibra $\left(\pi^{-1}(0), 0\right)$ sob o isomorfismo $i:\left(X_{0}, 0\right) \longrightarrow$ $\left(\pi^{-1}(0), 0\right)$.

Definição 4.1.4. Sejam $\pi:(X, 0) \longrightarrow(S, 0)$ e $\pi^{\prime}:\left(X^{\prime}, 0\right) \longrightarrow(S, 0)$ duas deformações de $\left(X_{0}, 0\right)$, de mesma base $(S, 0)$ e denotemos por $i$ e $i^{\prime}$ os mergulhos de $\left(X_{0}, 0\right)$ em $(X, 0) e$ $\left(X^{\prime}, 0\right)$, respectivamente. Um morfismo entre $\pi$ e $\pi^{\prime}$ é um morfismo $g:(X, 0) \longrightarrow\left(X^{\prime}, 0\right)$ tal que $\pi^{\prime} \circ g=\pi$ e $g \circ i=i^{\prime}$.

Definição 4.1.5. Seja $\pi:(X, 0) \longrightarrow(S, 0)$ uma deformação de $\left(X_{0}, 0\right)$ e $g:(T, 0) \longrightarrow(S, 0)$ uma aplicação analítica. A deformação induzida é uma aplicação plana $g^{*}(\pi):\left(X \times{ }_{S} T, 0\right) \longrightarrow$ $(T, 0)$.

Definição 4.1.6. Uma deformação $\pi:(X, 0) \longrightarrow(S, 0)$ é chamada versal ou semi- universal se toda deformação $\rho:(Y, 0) \longrightarrow(T, 0)$ de $\left(X_{0}, 0\right)$ é isomorfa à deformação $g^{*}(\pi)$, para alguma aplicação analítica $g:(T, 0) \longrightarrow(S, 0)$.

Podemos descrever as deformações semiuniversais explicitamente usando o espaço vetorial das deformações de primeira ordem. Denotamos por $\operatorname{Der}_{\mathbb{C}}\left(\mathcal{O}_{\mathbb{C}^{r}}\right)$ o módulo dos germes de campos de vetores analíticos na origem.

Definição 4.1.7. Sejam $\left(X_{0}, 0\right) \subset\left(\mathbb{C}^{r}, 0\right)$ o germe de uma variedade analítica complexa, com singularidade isolada na origem e $\mathcal{O}_{\left(X_{0}, 0\right)}=\mathcal{O}_{r} /$ I seu anel local.

\footnotetext{
${ }^{1} \mathrm{O}$ produto tensorial $\widehat{\otimes}$ é o produto tensorial usual e a notação "^"é para enfatizar que estamos considerando a graduação dos elementos envolvidos.
} 
a) O módulo normal de $\left(X_{0}, 0\right)$ é dado por

$$
\mathcal{N}_{\left(X_{0}, 0\right)}:=\operatorname{Hom}_{\mathcal{O}_{X_{0}, 0}}\left(I / I^{2}, \mathcal{O}_{\left(X_{0}, 0\right)}\right) .
$$

b) O espaço vetorial de dimensão finita das deformações de primeira ordem de $\left(X_{0}, 0\right)$ é isomorfo à $T_{\left(X_{0}, 0\right)}^{1}$ definido pela seguinte seqüência exata:

$$
\begin{aligned}
0 \longrightarrow \operatorname{Der}_{\mathbb{C}}\left(\mathcal{O}_{\mathbb{C}^{r}}\right) \longrightarrow \mathcal{O}_{\left(X_{0}, 0\right)} \otimes \operatorname{Der}_{\mathbb{C}}\left(\mathcal{O}_{\mathbb{C}^{r}}\right) \stackrel{\Theta}{\longrightarrow} \mathcal{N}_{\left(X_{0}, 0\right)} \longrightarrow T_{\left(X_{0}, 0\right)}^{1} \longrightarrow 0 \\
\frac{\partial}{\partial x_{i}} \longmapsto\left(f_{j} \longmapsto \frac{\partial f_{j}}{\partial x_{i}}\right),
\end{aligned}
$$

isto é, $T_{X}^{1}$ é dado pelo cokernel da aplicação $\Theta$.

Assim, dados $X_{0} \subset \mathbb{K}^{r}$ um germe de variedade definida por $\left(f_{1}, \ldots, f_{k}\right) \in \mathcal{P}$ e $a_{1}, . ., a_{r}$ são elementos do módulo normal de $X_{0}$, então uma deformação de $X_{0}$ é dada por

$$
F_{i}(\underline{x}, \underline{t})=f_{i}(\underline{x})+\sum_{j=1}^{r} t_{j} a_{j} .
$$

Definição 4.1.8. Dizemos que uma deformação $F=\left(F_{1}, \ldots, F_{k}\right)$ é trivial se existe um automorfismo $\varphi(x, \epsilon)$ de $\mathbb{C}^{r} \times \operatorname{Hom}_{\mathcal{O}_{X_{0}, 0}}\left(I / I^{2}, \mathcal{O}_{\left(X_{0}, 0\right)}\right)$ tal que $F$ e $f \circ \varphi$ determinam o mesmo ideal.

Uma singularidade é rígida (ou estável) se toda deformação $\pi:(X, 0) \longrightarrow(S, 0)$ de $\left(X_{0}, 0\right)$ é isomorfa à deformação trivial $p_{0}:\left(X_{0} \times S, 0\right) \longrightarrow(S, 0)$.

\subsection{Deformações de Germes Cohen-Macaulay de Codi- mensão 2}

A seguinte proposição é uma aplicação do Teorema de Hilbert-Burch e descreve os germes Cohen-Macaulay de codimensão 2 e suas deformações mostrando a correspondência entre o espaços das deformações de primeira ordem da variedade e o espaço das deformações da matriz de representação, justificando o uso da $\mathcal{G}$-equivalência de matrizes para o estudo destes germes.

Proposição 4.2.1. ([16], pág. 3994).

1) Sejam $M$ uma matriz $(n+1) \times n$ com entradas em $\mathcal{O}_{r}$ e $f=\left(f_{1}, \ldots, f_{n+1}\right)$ seus menores maximais e, por abuso de notação, o ideal destes menores. Se codim $(V(f))$ $\geq 2$, a seguinte seqüência

$$
0 \longrightarrow \mathcal{O}_{r}^{n} \longrightarrow \mathcal{O}_{r}^{n+1} \longrightarrow \mathcal{O}_{r} /(f) \longrightarrow 0
$$


é exata. A álgebra quociente $\mathcal{O}_{r} /(f)$ é Cohen-Macaulay e codim $(V(f))=2$.

2) Se $X \subset \mathbb{C}^{r}$ é Cohen-Macaulay, codim $(X)=2$ e $X=V(I)$, então $\mathcal{O}_{r} /$ I tem uma resolução minimal do tipo

$$
0 \longrightarrow \mathcal{O}_{r}^{n} \longrightarrow \mathcal{O}_{r}^{n+1} \longrightarrow \mathcal{O}_{r} / I \longrightarrow 0 .
$$

Além disso, existe um elemento invertível $u \in \mathcal{O}_{r}$ tal que $I=u \cdot f$, onde $f$ é novamente o ideal dos menores maximais de $M$.

3. Qualquer deformação de $M$ é uma deformação de $X$;

4. Qualquer deformação de $X$ pode ser gerada por uma perturbação da matriz $M$.

Segue deste resultado que qualquer deformação de uma variedade Cohen-Macaulay de codimensão 2 pode ser dada como uma perturbação da matriz de representação. Vamos mostrar que podemos expressar os módulos $\mathcal{N}_{X}$ e $T_{1}$ em termos de matrizes e, dessa forma, podemos tratar a base de deformação semi-universal usando matrizes de representação.

Proposição 4.2.2. ([16], pág. 3996) Seja $M$ uma matriz $(n+1) \times n$ com entradas no ideal maximal de $\mathbb{C}\left\{x_{1}, \ldots, x_{r}\right\}$ e $X$ o germe definido por seus menores maximais. O módulo normal é dado por

$$
\mathcal{N}_{X} \cong \frac{\operatorname{Mat}_{(n+1, n)}\left(\mathcal{O}_{r}\right)}{\operatorname{Im}(g)}
$$

onde $g$ é a aplicação definida em 2.6.

\section{Demonstração.}

Para obter o resultado do lema é suficiente provar que a seqüência

$$
\operatorname{Mat}_{(n+1, n+1)}\left(\mathcal{O}_{r}\right) \oplus \operatorname{Mat}_{(n, n)}\left(\mathcal{O}_{r}\right) \stackrel{g}{\longrightarrow} \operatorname{Mat}_{(n+1, n)}\left(\mathcal{O}_{r}\right) \stackrel{\phi}{\longrightarrow} \mathcal{N}_{X} \longrightarrow 0
$$

é exata.

Passo 1: Definição de $\phi$ e prova da sua sobrejetividade.

Um elemento $b \in \mathcal{N}$ é usualmente identificado com uma deformação $\left(\Delta_{1}=\epsilon b_{1}, \ldots, \Delta_{n+1}=\right.$ $\left.\epsilon b_{n+1}\right)$. Se $M+\epsilon N$ é uma perturbação, a correspondente deformação de primeira ordem é dada pelos seus menores maximais

$$
\Delta_{k}+\epsilon \sum_{i=1}^{n+1} \sum_{j=1}^{n}(-1)^{i+j} N_{i j} M_{k ; i j}, \quad \forall 1 \leq k \leq n+1,
$$

onde $M_{k ; i j}$ é o menor submaximal de $M$ obtido retirando a $i$-ésima e a $k$-ésima colunas e a $j$-ésima linha e então formando o determinante. 
Como estamos considerando deformações de primeira ordem, isto é, calculando módulo $\epsilon^{2}$, nenhum termo pode conter mais que uma entrada de $N$ como fator.

Assim, podemos definir $\phi$ por

$$
\begin{aligned}
\phi: \operatorname{Mat}_{(n+1, n)}\left(\mathcal{O}_{r}\right) & \longrightarrow \mathcal{N}_{X} \\
N & \longmapsto\left(\delta_{k} \longmapsto \sum_{i=1}^{n+1} \sum_{j=1}^{n}(-1)^{i+j} N_{i j} M_{k ; i j} \forall 1 \leq k \leq n+1\right) .
\end{aligned}
$$

Ainda usando a caracterização de germes Cohen-Macaulay, sempre existe uma matriz de representação para qualquer deformação. Portanto, $\phi$ é sobrejetiva.

Passo 2: Complexo em $\operatorname{Mat}_{(n+1, n)}\left(\mathcal{O}_{r}\right)$.

Considere $\tilde{M}=\tilde{A} M+M \tilde{B}$ onde $\tilde{A} \in \operatorname{Mat}\left(n+1, n+1 ; \mathcal{O}_{r}\right)$ e $\tilde{B} \in \operatorname{Mat}\left(n, n ; \mathcal{O}_{r}\right)$ são perturbações de $A$ e $B$. Como anteriormente, $\Delta$ denota os menores maximais de $M, \Delta_{\epsilon}=$ $\left(\Delta_{\epsilon_{1}}, \ldots, \Delta_{\epsilon_{(n+1)}}\right)$ os menores de $M+\epsilon \tilde{M}$.

Então, obtemos a seguinte igualdade para o menor maximal de $M+\epsilon \widetilde{M}$, obtido eliminandose a $k$ - ésima coluna:

$$
\begin{aligned}
\Delta_{\epsilon k} & =\operatorname{det}\left(M_{i j}+\sum_{l} \epsilon A_{i l} M_{l j}+\sum_{l} \epsilon M_{i l} B_{l j}\right)_{i \in\{1, \ldots, k-1, k+1, \ldots, n+1\} ; j \in\{j \in\{1, \ldots, n\}\}}= \\
& =\left(1+\epsilon \sum_{i=1}^{n+1} A_{i i}+\epsilon \sum_{i=1}^{n} B_{i i}\right) \Delta_{k}+\epsilon \sum_{i=1}^{n+1} \Delta_{i k} \Delta_{i} .
\end{aligned}
$$

Assim, $\Delta_{\epsilon}$ e $\Delta$ geram o mesmo ideal em $\frac{\mathbb{C}[\epsilon]}{\left(\epsilon^{2}\right)\left\{x_{1}, \ldots, x_{r}\right\}}$.

Passo 3: Mostrar que a seqüência é exata em $\operatorname{Mat}_{(n+1, n)}\left(\mathcal{O}_{r}\right)$, isto é, $\operatorname{Im}(g)=\operatorname{Ker}(\phi)$.

Seja $\tilde{M}$ um elemento do $\operatorname{Ker}(\phi), \Delta$ e $\delta_{\epsilon}$ os ideais gerados pelos menores maximais de $M$ e $M+\epsilon \tilde{M}$. Como $\tilde{M}$ está no kernel de $\phi$, os ideais $\delta$ e $\Delta_{\epsilon}$ são iguais conseqüentemente, obtemos uma $\mathcal{R}$-aplicação linear $A$, onde $\mathcal{R}=\frac{\mathbb{C}[\epsilon]}{\left(\epsilon^{2}\right)\left\{x_{1}, \ldots, x_{r}\right\}}$ e o seguinte diagrama é comutativo

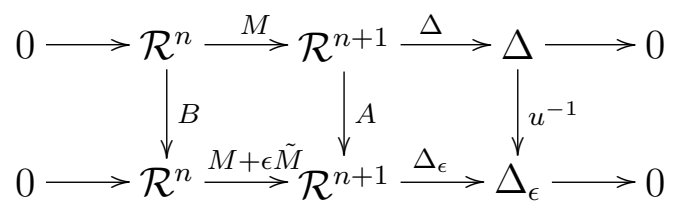

Assim, $B$ é um isomorfismo. Além disso, sabemos que

$$
\left.A^{-1}\right|_{\epsilon=0}=\left.I d_{n+1} \quad B\right|_{\epsilon=0}=I d_{n},
$$

pois, $M=M-\left.\epsilon \tilde{M}\right|_{\epsilon=0}$. Portanto,

$$
M=A^{-1}(M-\epsilon \tilde{M}) B=\left(I d_{n+1}+\epsilon \tilde{A}\right)(M-\epsilon \tilde{M})\left(I d_{n}+\epsilon \tilde{B}\right)=M-\epsilon \tilde{M}+\epsilon \tilde{A} M+\epsilon M \tilde{B} .
$$


Proposição 4.2.3. Com as mesmas notações do lema anterior

$$
T_{X}^{1}=\frac{\operatorname{Mat}_{(n+1, n)}\left(\mathcal{O}_{r}\right)}{J(M)+\operatorname{Im}(g)}
$$

onde

$$
J(M)=\left(\begin{array}{ccc}
\frac{\partial M_{11}}{\partial x_{j}} & \cdots & \frac{\partial M_{1 n}}{\partial x_{j}} \\
\vdots & \vdots & \vdots \\
\frac{\partial M_{(n+1) 1}}{\partial x_{j}} & \cdots & \frac{\partial M_{(n+1) n}}{\partial x_{j}}
\end{array}\right) \forall 1 \leq j \leq r .
$$

\section{Demonstração.}

Por definição $T_{X}^{1}$ é o cokernel de

$$
\begin{aligned}
\psi: \frac{\mathcal{O}_{r}}{(\Delta)} \otimes \operatorname{Der}_{\mathbb{C}}\left(\mathcal{O}_{\mathbb{C}^{r}}\right) & \longrightarrow \mathcal{N}_{X} \\
\frac{\partial}{\partial x_{i}} & \longmapsto\left(\frac{\partial \Delta_{1}}{\partial x_{i}}, \ldots, \frac{\partial \Delta_{n+1}}{\partial x_{i}}\right) .
\end{aligned}
$$

Por outro lado, pelas regras de diferenciação

$$
\frac{\partial \Delta_{k}}{\partial x_{i}}=\sum_{l=1}^{n+1} \sum_{j=1}^{n}(-1)^{l+j} \frac{\partial M_{l j}}{\partial x_{i}} M_{k ; j l}
$$

e é assim a $k$-ésima componente de $\phi\left(\left(\frac{\partial M_{l j}}{\partial x_{i}}\right)_{1 \leq l \leq n+1}\right)$ onde $\phi$ é a aplicação definida no lema anterior.

Conhecendo isso, podemos completar a demonstração colocando $\psi$ em notação matricial

$$
\begin{aligned}
\psi: \frac{\mathcal{O}_{r}}{(\Delta)} \otimes \operatorname{Der}_{\mathbb{C}}\left(\mathcal{O}_{\mathbb{C}^{r}}\right) & \longrightarrow \mathcal{N}_{X} \\
\frac{\partial}{\partial x_{i}} & \longmapsto\left(\frac{\partial M_{l j}}{\partial x_{i}}\right)_{1 \leq l \leq n+1} 1 \leq j \leq n
\end{aligned}
$$

Como qualquer perturbação da matriz de representação define uma perturbação da variedade, não existem obstruções para levantamentos de deformações de primeira ordem para deformações de ordem mais altas e assim, a base do espaço das deformações semi-universais é suave.

\section{$4.3 \quad \mathcal{G}$-Estabilidade de Matrizes}

Dada $X$ uma variedade determinantal, Cohen-Macaulay de codimensão 2, com singularidade isolada, estamos interessados em determinar condições para que perturbações estáveis de $X$ 
sejam variedades suaves. A condição de estabilidade é equivalente à transversalidade de $M$ com relação aos estratos da estratificação de $\operatorname{Mat}_{(n, n+1)}(\mathbb{C})$ (ver Proposição 2.4.1). Ou seja, $X$ é suave se, e somente se, a matriz de representação não intercepta o conjunto $\Delta$ das matrizes singulares. Como a codimensão de $\Delta$ é igual a 2 , isto ocorre se $r<6$.

M. Schaps estudou deformações de esquemas determinantais obtidos a partir de sua matriz de representação usando técnicas algébricas aplicadas à teoria de deformações (ver [41], [42] e [43]). Em [42], a autora mostra que, no caso especial de deformações de curvas e esquemas Cohen- Macaulay de codimensão 2, é possível obter sob certas condições, deformações planas cujas fibras genéricas são não singulares. Em [41], a autora generaliza este resultado para um esquema algébrico afim determinantal. Os mesmos resultados foram obtidos por D. Laksov em [34] usando teoremas gerais de transversalidade de aplicações.

Vamos aqui estudar o caso de existência de perturbações estáveis de variedades CohenMacaulay de codimensão 2, com singularidade isolada na origem, do ponto de vista da $\mathcal{G}$ equivalência.

Proposição 4.3.1. Seja $M$ uma matriz $n \times(n+1)$ cujas entradas estão no ideal maximal de $\mathcal{O}_{r}$. Se $r<n(n+1)$, então $M$ não é $\mathcal{G}$-estável.

\section{Demonstração.}

Como $m_{i j}(0)=0$ para todo $i, j$, então as matrizes constantes só podem ser obtidas no espaço tangente da parte $\left\{\frac{\partial M}{\partial x_{i}}\right\}$. Segue que podemos obter no máximo $r$ matrizes constantes linearmente independentes. Mas $r<n(n+1)$, então $T \mathcal{G} M \neq \operatorname{Mat}_{(n, n+1)}\left(\mathcal{O}_{r}\right)$ e, portanto, $M$ não pode ser $\mathcal{G}$ estável.

Assim, se $M$ é $\mathcal{G}$ - estável e $r<n(n+1)$, então $M(0) \neq 0$. A próxima proposição, caracteriza as variedades Cohen- Macaulay, de codimensão dois, com singularidade isolada na origem e que são $\mathcal{G}$ - estáveis.

Proposição 4.3.2. Seja $M$ uma matriz $2 \times 3$, com entradas no ideal maximal de $\mathcal{O}_{r}$ e $r \geq 6$. Então, $M$ é $\mathcal{G}$ - estável se, e somente se, $M$ é $\mathcal{G}$-equivalente a $M^{\prime}=\left(\begin{array}{lll}x_{1} & x_{2} & x_{3} \\ x_{4} & x_{5} & x_{6}\end{array}\right)$.

\section{Demonstração.}

Suponhamos inicialmente que $M$ tem 1- jato da forma $M^{\prime}$, então $M=M^{\prime}+P$, onde $P=\left(p_{i j}(x)\right) \in \mathfrak{M}^{2}$. Assim,

$$
T \mathcal{G} M+\mathfrak{M} \operatorname{Mat}_{(2,3)}\left(\mathcal{O}_{r}\right)=\operatorname{Mat}_{(2,3)}\left(\mathcal{O}_{r}\right)
$$


Aplicando o Lema de Nakayama, obtemos que $T \mathcal{G} M=\operatorname{Mat}_{2,3}\left(\mathcal{O}_{r}\right)$ e, portanto, $M$ é $\mathcal{G}$ - estável.

Reciprocamente, se $M$ é $\mathcal{G}$ - estável e $M(0)=0$, então, $T \mathcal{G} M=\operatorname{Mat}_{(2,3)}\left(\mathcal{O}_{r}\right)$. Em particular, $\operatorname{TGM}(0)=\operatorname{Mat}_{(2,3)}(\mathbb{C})$, isto é,

$$
\mathbb{C}\left\{\frac{\partial M}{\partial x_{i}}\right\}=\operatorname{Mat}_{(2,3)}(\mathbb{C})
$$

Portanto, é fácil ver que a menos de mudanças de coordenadas $M=M^{\prime}$.

Proposição 4.3.3. Seja $\mathcal{O}_{1}=\mathbb{C}\{x\}$ e $M \in \operatorname{Mat}_{(2,3)}\left(\mathcal{O}_{1}\right)$. Então, $M$ é $\mathcal{G}$ - estável se, e somente se, o posto de $M$ é igual a dois.

\section{Demonstração.}

Suponhamos inicialmente que $M$ seja $\mathcal{G}$ - estável, então

$$
\operatorname{TGM}(0)=\operatorname{Mat}_{(2,3)}(\mathbb{C}) .
$$

Observamos que neste caso a parte $\left\{\frac{\partial M}{\partial x}(0)\right\}$ contribui com no máximo uma matriz constante para o espaço tangente. Assim, o conjunto $\left\{R_{i j} M(0), C_{l k}(M)(0)\right\}$ deve ser formado por cinco matrizes constantes linearmente independentes. Agora, isso só é possível se o posto de $M$ for igual a 2 .

Reciprocamente, se $M$ tem rank 2, então $M(0)$ é $\mathcal{G}$ - equivalente a uma matriz da forma

$$
\left(\begin{array}{lll}
1 & 0 & 0 \\
0 & 1 & 0
\end{array}\right)
$$

então, $T \mathcal{G} M+\mathfrak{M M a t}_{(2,3)}\left(\mathcal{O}_{r}\right)=\operatorname{Mat}_{(2,3)}\left(\mathcal{O}_{r}\right)$. Portanto, $M$ é $\mathcal{G}$ - estável.

Proposição 4.3.4. Se X é uma variedade Cohen-Macaulay de codimensão 2, com singularidade isolada na origem, definida pelos menores $n \times n$ de uma matriz $M \in \operatorname{Mat}_{(n, n+1)}\left(\mathcal{O}_{r}\right)$, onde $\mathcal{O}_{r}=\mathbb{C}\left\{x_{1}, \cdots, x_{r}\right\}$, com $r<6$ e $\mathcal{G}_{e^{-}}$codimensão de $M$ finita. Então, uma estabilização de $X$ existe e é única a menos de difeomorfismos.

\section{Demonstração.}

Sejam $M^{\prime}$ a deformação versal de $M, \mathfrak{X} \subset \mathbb{C}^{r} \times \mathbb{C}^{k}$ o espaço das deformações versais de $X$ e

$$
\operatorname{Bif}(\mathfrak{X})=\left\{u \in \mathbb{C}^{k} \mid X_{u} \subset \mathfrak{X} \text { não é estável }\right\}
$$


o seu conjunto de bifurcação. Observemos que o conjunto Bif(X) é um conjunto analítico próprio e, portanto, seu complementar é um conjunto conexo. Além disso, se $u$ é um elemento que está no complementar do conjunto $\operatorname{Bif}(\mathfrak{X})$ e $M^{\prime}$ é a matriz que define $X_{u}$, então $M^{\prime}$ é $\mathcal{G}$ estável. Mas se $M^{\prime}$ é $\mathcal{G}$-estável, sabemos que $M^{\prime}(0) \neq 0$ e, portanto, existe $U$ vizinhança da origem em $\mathbb{C}^{r}$ tal que a singularidade definida por $M^{\prime}$ é suave. Portanto, $M^{\prime}$ é um suavização de M. A unicidade do suavização segue do fato que o conjunto $(B i f(\mathfrak{X}))^{c}$ é um conjunto conexo.

Exemplo 4.3.1. Considere a variedade $X$ determinada pelo anulamento dos menores maximais de uma matriz $M=\left(m_{i j}\right)_{n \times n+1}$, da forma

$$
\left(\begin{array}{ll}
R & 0 \\
0 & I
\end{array}\right)
$$

onde $R=\left(r_{i j}\right)$ é uma matriz $2 \times 3$ com entradas no ideal maximal de $\mathcal{O}_{6}$ e $I$ é a matriz identidade de ordem $n-2$. Observemos que $X$ tem singularidade isolada. É possível verificar, usando um resultado de T. Svanes (ver [41], exemplo 3), que um membro genérico de qualquer família plana que deforma $X$ também tem singularidade isolada. Logo, a desigualdade da proposição anterior deve ser estrita. 


\section{Capítulo 5}

\section{A Fibra de Milnor}

Neste capítulo, descrevemos alguns resultados sobre a topologia de variedades analíticas complexas com singularidade isolada, tais como a definição de bola de Milnor e de suavização. Além disso, apresentamos alguns resultados já conhecidos sobre a existência de suavização e a topologia da fibra genérica. Gostaríamos de agradecer ao professor Osamu Saeki pelas importantes sugestões que tornaram possíveis as demonstrações dos resultados deste Capítulo.

Na Proposição 5.2.1, usamos a Teoria de Morse para descrever o tipo de homotopia da fibra genérica da suavização de uma tal variedade. As principais referências deste capítulo são [24], [51] e [36].

\subsection{Suavizações e Bola de Milnor}

Seja $X_{0} \subset \mathbb{C}^{r}$ um germe de variedade analítica, $d$-dimensional com singularidade isolada na origem. Uma suavização de $X_{0}$ é uma deformação plana de $X_{0}$ cujas fibras próximas são suaves.

Definição 5.1.1. Dizemos que um germe de variedade analítica $\left(X_{0}, 0\right)$ com singularidade isolada de dimensão complexa $n \geq 1$ admite suavização, se existem uma bola aberta $B_{\epsilon}(0) \subset \mathbb{C}^{r}$ com centro em zero, um subespaço fechado $X \subset B_{\epsilon}(0) \times D$, onde $D \subset \mathbb{C}$ é um disco aberto com centro em zero e uma aplicação analítica própria

$$
F: X \longrightarrow D
$$

que é a restrição da projeção $p: B \times D \longrightarrow D$ tal que

a) Fé plana;

b) $\left(F^{-1}(0), 0\right)$ é isomorfo a $\left(X_{0}, 0\right)$; 
c) $F^{-1}(t)$ é não singular para $t \neq 0$.

Segue da definição acima que $X$ tem singularidade isolada em zero e é normal se $X_{0}$ é normal em zero. Além disso,

$$
\left.F\right|_{F^{-1}(D-0)}: F^{-1}(D-0) \longrightarrow D-\{0\}
$$

é um fibrado com fibras não singulares $X_{t}=F^{-1}(t)$.

Definição 5.1.2. Seja $\left(X_{0}, 0\right)$ um germe de singularidade isolada complexa n-dimensional. Dado $\epsilon>0$, dizemos que a bola aberta $B_{\epsilon}(0) \subset \mathbb{C}^{r}$ é uma bola de Milnor para $\left(X_{0}, 0\right)$ se as seguintes condições são verificadas:

i) Existe um representante de $\left(X_{0}, 0\right)$ definido em $\bar{B}_{\epsilon}(0)$ que intercepta $\partial \bar{B}_{\epsilon}(0)$ transversalmente nos pontos suaves;

ii) $X_{0}-\{0\}$ é não singular e intercepta $\partial \bar{B}_{\epsilon^{\prime}}(0)$ para toda $B_{\epsilon^{\prime}}(0) \subset B_{\epsilon}(0)$.

Notemos que se $B_{\epsilon}(0)$ é uma bola de Milnor para $\left(X_{0}, 0\right)$, então para todo $\epsilon^{\prime}<\epsilon, B_{\epsilon^{\prime}}(0)$ é também uma bola de Milnor para $\left(X_{0}, 0\right)$ e $X_{0}$ é contrátil em 0 .

J. Milnor mostra em [36] o seguinte resultado que afirma que qualquer germe de singularidade isolada admite uma pequena bola de Milnor.

Corolário 5.1.1. ([36], pág. 17) Para todo $\epsilon$ suficientemente pequeno, $B_{\epsilon}(0)$ intercepta $X_{0}$ em uma variedade suave.

Sejam $F: X \longrightarrow D$ uma suavização de $\left(X_{0}, 0\right)$ definida em uma vizinhança de $\bar{B}_{\epsilon}(0) \times D$, e $B_{\epsilon}(0)$ uma bola de Milnor para $\left(X_{0}, 0\right)$. Então se $D$ é suficientemente pequeno vale

iii) A fibra de $F$ em $t$ intercepta $\partial \bar{B}_{\epsilon}(0) \times\{t\}$ transversalmente para todo $t \in D$.

Denotamos por $\bar{X}$ e $\bar{X}_{t}$ os fechos de $X$ e $X_{t}$ em $\bar{B}_{\epsilon}(0) \times D$, então as condições $\left.i\right)$, ii) e $\left.i i i\right)$ implicam que $\bar{X}_{0}-\{0\}$ é uma variedade cuja fronteira, $\partial \bar{X}_{0}$, é difeomorfa à fronteira de $X_{t}$, para todo $t \in D-\{0\}$. Além disso, pelo teorema de Fibração de Ehresmann

$$
F: \bar{X}-\bar{X}_{0} \longrightarrow D-\{0\}
$$

é um fibrado $C^{\infty}$ localmente trivial.

Definição 5.1.3. Se $B_{\epsilon}(0)$ e $D$ são escolhidos de tal maneira que as condições i) - iii) se verifiquem, então $B \times D$ é chamado tubo de Milnor e $X_{t}=F^{-1}(t), t \neq 0$, são as fibras de Milnor da suavização. 
Dado $(X, 0)$ um germe de variedade, um problema importante é determinar em que condições temos a existência e unicidade de suavização para $(X, 0)$.

Sabemos que no caso de hipersuperfícies e intersecções completas sempre existe uma suavização única pois o espaço base de suas deformações semiuniversais é conexo. Contudo, existem singularidades que não admitem suavização e outras singularidades de superfícies que admitem suavização, mas que para diferentes suavizações a característica de Euler das fibras são distintas $([51])$.

Para singularidades determinantais, a existência e unicidade da suavização não se verificam em geral. Mas vale o seguinte resultado:

Teorema 5.1.1. ([51], pág. 241.) Suponha que $(X, 0)$ é determinantal com singularidade isolada definida por menores $t \times t$ de uma matriz $M, n \times p$, com entradas em $\mathcal{O}_{r}$ com $2 \leq t \leq$ $n \leq p$. Se $\operatorname{dim}(X)<n+p-2 t+3$, então existe uma suavização para $X$.

Em particular, se $(X, 0)$ é Cohen-Macaulay com codimensão menor ou igual a 2 e $\operatorname{dim}(X, 0) \leq$ 3 , então $(X, 0)$ admite uma suavização. Observemos ainda que para singularidades CohenMacaulay, de codimensão menor ou igual a 2 não existem obstruções para o levantamento para deformações de segunda ordem, a base das deformações semiuniversais é suave ([16]).

Para curvas reduzidas, a topologia da fibra genérica já foi bastante estudada ([6]). Por exemplo temos o seguinte resultado:

Teorema 5.1.2. ([6].) Seja $f: Y \longrightarrow D$ um bom representante de uma família plana $f:$ $(Y, 0) \longrightarrow(D, 0)$ de curvas reduzidas. Então, para todo $t \in D$ a fibra $Y_{t}$ é conexa.

De maneira geral temos o seguinte resultado obtido por Greuel e Steenbrink em [24].

Teorema 5.1.3. ([24], pág. 17.) Sejam (X,0) um espaço analítico complexo, $n$-dimensional com singularidade isolada e $X_{t}$ a fibra de Milnor de uma suavização de $(X, 0)$. Então, $\Pi_{i}\left(X_{t}\right)=$ 0 para $i \leq \operatorname{dim} X-\operatorname{codim} X$.

Corolário 5.1.2. Se $(X, 0) \subset\left(\mathbb{C}^{r}, 0\right)$ é uma variedade Cohen-Macaulay de codimensão 2 com singularidade isolada, então sua fibra de Milnor é (dimX - 2)- conexa.

Denotemos por $b_{i}\left(X_{t}\right)$ o $i$ - ésimo número de Betti de $X_{t}$.

Teorema 5.1.4. ([24], pág. 540) Seja $X_{t}$ uma fibra de Milnor de uma singularidade normal, então $b_{1}\left(X_{t}\right)=0$. 


\subsection{A Topologia de Variedades com Singularidade Iso- lada}

Seja $(X, 0) \subset\left(\mathbb{C}^{r}, 0\right)$ uma variedade $d$ - dimensional com singularidade isolada na origem. Suponhamos que exista uma suavização para $X$, isto é, uma família plana

$$
\Pi: \mathfrak{X} \longrightarrow D \subset \mathbb{C}
$$

restrição da projeção $B_{\epsilon}(0) \times D \longrightarrow D$, tal que $X_{t}=\Pi^{-1}(t)$ é suave para $t \neq 0$ e $X_{0}=X$.

Notemos que $\mathfrak{X}$ também tem singularidade isolada na origem. Sejam $p$ uma função analítica complexa definida em $X$, com singularidade isolada na origem. Seja

$$
\begin{aligned}
\tilde{p}: \mathfrak{X} \subset \mathbb{C}^{r} \times \mathbb{C} & \longrightarrow \mathbb{C} \\
(x, t) & \longrightarrow \widetilde{p}(x, t),
\end{aligned}
$$

tal que $\widetilde{p}(x, 0)=p(x)$ e tal que para todo $t \neq 0, \widetilde{p}(\cdot, t)=p_{t}$ seja uma função de Morse em $X_{t}$ (ver Teorema 1.2.1).

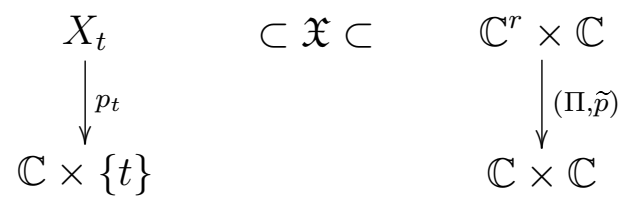

Notemos que o número de pontos críticos de $p_{t}$ é finito. De fato, $x$ é um ponto crítico de $p_{t}$ se, e somente se, $x$ é um ponto crítico da função $R e\left(p_{t}\right): X_{t} \longrightarrow \mathbb{R}$. Como a parte real de $p_{t}$ é uma função algébrica em $X_{t}$, o número de pontos críticos de $R e\left(p_{t}\right)$ e, conseqüentemente de $p_{t}$, é finito.

Proposição 5.2.1. Sejam $X$ uma variedade d-dimensional com singularidade isolada na origem que admite uma suavização $\Pi e, p_{t}: X_{t} \longrightarrow \mathbb{C}, p_{t}=\widetilde{p}(\cdot, t)$ como acima. Então,

a) $S e t \neq 0$

$$
X_{t} \simeq p_{t}^{-1}(0) \dot{\cup}\{\text { células de dimensão } d\},
$$

onde $\dot{\cup}$ indica a colagem dos dois espaços $e \simeq$ indica que os espaços têm mesmo tipo de homotopia.

b)

$$
\mathcal{X}\left(X_{t}\right)=\mathcal{X}\left(\left(p_{t}\right)^{-1}(0)\right)+(-1)^{d} n_{\sigma},
$$

onde $n_{\sigma}$ é o número de pontos críticos de $p_{t}$ e $\mathcal{X}\left(X_{t}\right)$ é a característica de Euler de $X_{t}$. 


\section{Demonstração.}

Sejam $x_{1}, \ldots, x_{\nu}$ os pontos críticos de $p_{t}$ e $y_{i}=p_{t}\left(x_{i}\right), 1 \leq i \leq \nu$, seus valores críticos. Suponhamos ainda que 0 seja um valor regular de $p_{t}$, para todo $t \neq 0$. Denotemos por $E_{i}$ os segmentos que ligam os pontos $y_{i}$ à 0 que têm somente $0=p_{t}(0)$ como ponto em comum e por $E$, a união destes segmentos. Tome $\eta>0$ suficiente pequeno tal que $y_{i} \in D_{\eta}(0)$ para todo $1 \leq i \leq \nu$.
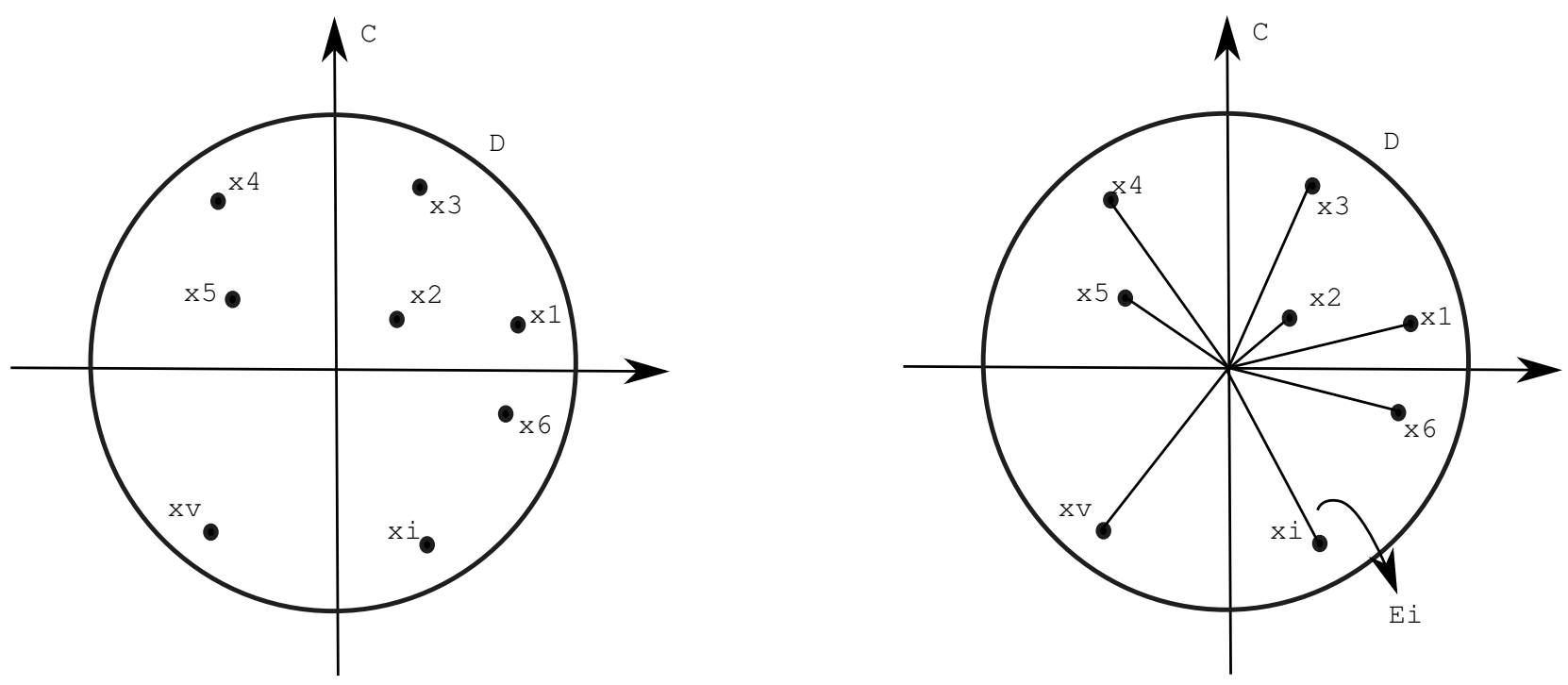

Figura 5.1:

Observemos que $D_{\eta}(0)$ é uma vizinhança regular de $E$ que retrai em $E$. Podemos realizar
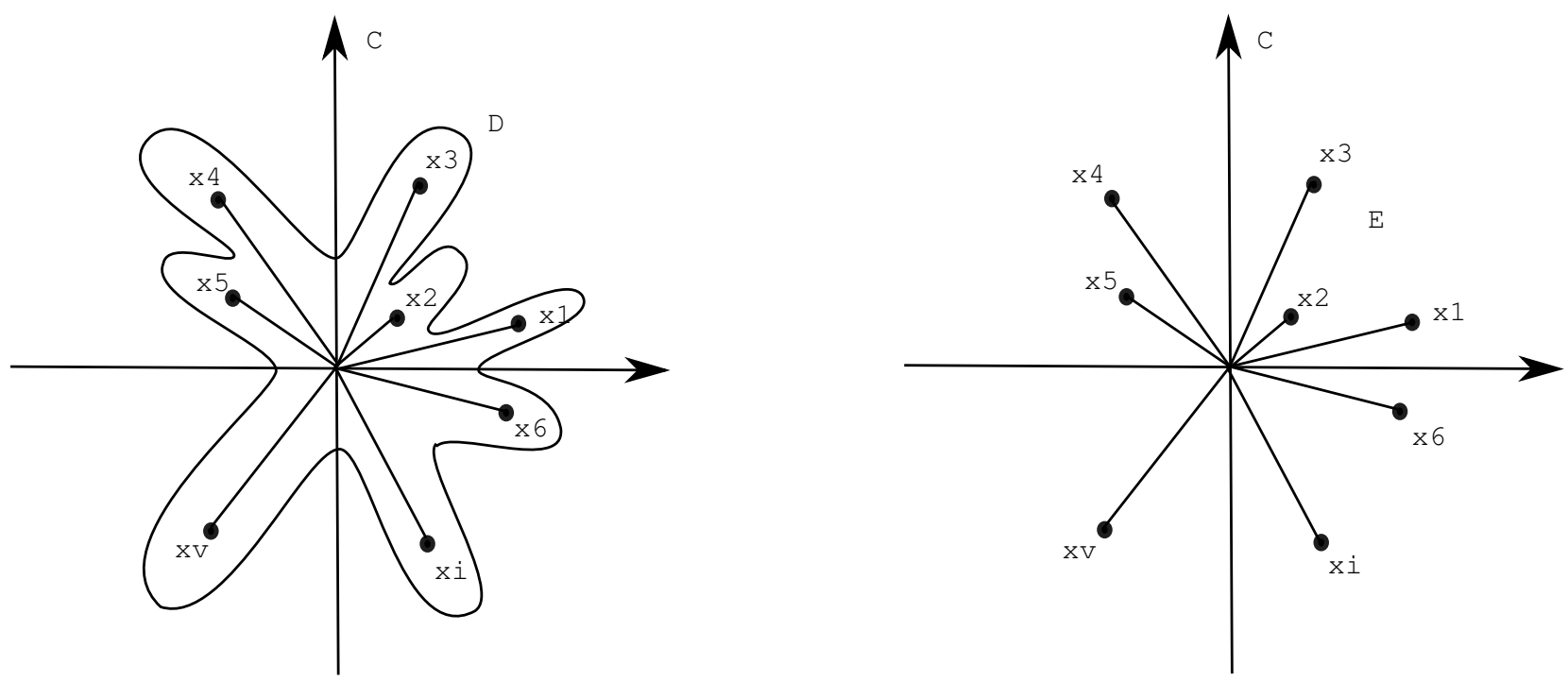

Figura 5.2:

esta retração através de um campo suave de vetores que pode ser levantado para o espaço $X_{t}$. 
Integrando este campo de vetores, o espaço $p_{t}^{-1}\left(D_{\eta}\right)$ retrai por deformação em $p_{t}^{-1}(E)$.

Assim,

$$
X_{t}=p_{t}^{-1}\left(D_{\eta}\right) \simeq p_{t}^{-1}(E)=p_{t}^{-1}\left(\bigcup_{i} E_{i}\right)=\bigcup_{i}\left(p_{t}^{-1}\left(E_{i}\right)\right)=p_{t}^{-1}(0) \cup \overline{\left(\bigcup_{i} p_{t}^{-1}\left(E_{i}-\{0\}\right)\right)}
$$

Notemos primeiramente que $x_{i}$ é ponto crítico da restrição de $p_{t}$ a $p_{t}^{-1}\left(E_{i}-\{0\}\right)$ se, e somente se, $x_{i}$ é ponto crítico da restrição da parte real de $p_{t}$ à $p_{t}^{-1}\left(E_{i}-\{0\}\right)$. Logo, segue da Teoria Clássica de Morse (ver [37]) que

$$
X_{t}=p_{t}^{-1}(0) \cup \overline{\left(\bigcup_{i} p_{t}^{-1}\left(E_{i}-\{0\}\right)\right)} \simeq p_{t}^{-1}(0) \dot{\cup}\{\text { células de dimensão } d\}
$$

onde $\dot{U}$ indica a colagem dos dois espaços $\mathrm{e} \simeq$ indica que os espaços têm mesmo tipo de homotopia.

Como a característica de Euler é um invariante homotópico, usando a decomposição (5.1) temos

$$
\mathcal{X}\left(X_{t}\right)=\mathcal{X}\left(\left(p_{t}\right)^{-1}(0)\right)+(-1)^{d} n_{\sigma},
$$

onde $n_{\sigma}$ é o número de pontos críticos de $p_{t}$.

Uma conseqüência da decomposição (5.1) é que somente $\overline{p_{t}^{-1}\left(E_{i}-\{0\}\right)}$ contribui para a parte livre de $H_{n}\left(X_{t}, \mathbb{Z}\right)$. Assim, obtemos o seguinte corolário:

Corolário 5.2.1. O d-ésimo número de Betti de $X_{t}$ é menor ou igual ao número de pontos críticos de $p_{t}$, isto é $b_{d}\left(X_{t}\right) \leq n_{\sigma}$. 


\section{Capítulo 6}

\section{Invariantes de Variedades Determinantais}

J. Milnor, em [36], obtém resultados importantes sobre a topologia de hipersuperfícies com singularidade isolada. As fibras de Milnor do germe de função analítica complexa $f:\left(\mathbb{C}^{r}, 0\right) \longrightarrow$ $(\mathbb{C}, 0)$, na vizinhança de um ponto singular isolado, têm o tipo de homotopia de um bouquet de esferas de dimensão real igual à dimensão complexa da fibra. O número de esferas deste bouquet é chamado número de Milnor de $f$ e denotado por $\mu(f)$.

O número de Milnor tem um papel fundamental na teoria de singularidades de hipersuperfícies e pode ser caracterizado de diversas maneiras. Em particular, $\mu(f)$ pode ser calculado algebricamente como $\operatorname{dim} \frac{\mathcal{O}_{r}}{J(f)}$, onde $J(f)$ é o ideal Jacobiano de $f$.

Os resultados de J. Milnor foram estendidos por H. Hamm para intersecções completas com singularidade isolada.

Um dos problemas atuais em teoria de Singularidades é a extensão do conceito de número de Milnor para classes mais gerais de conjuntos analíticos. Neste capítulo, consideramos esta questão para variedades Cohen-Macaulay de codimensão 2, com singularidade isolada na origem. O resultado principal deste capítulo é o Teorema 6.1.1, no qual obtemos uma fórmula do tipo Lê- Greuel para superfícies determinantais em $\mathbb{C}^{4}$. Na seção 6.2, consideramos a definição de índice de uma 1- forma diferenciável definida em uma variedade determinantal introduzida por W. Ebeling e S. M. Gusein-Zade e, comparamos os resultados de [13] com os resultados da seção 6.1. Na seção 6.3, calculamos o número de Milnor de algumas singularidades simples de superfícies em $\mathbb{C}^{4}$, classificadas por A. Frühbis-Krüger, A. Neumer em [18]. Com base nestes cálculos, mostramos que a relação $\tau(X)=\mu(X)+1$ se verifica para estes exemplos. 


\subsection{Variedades Determinantais}

Uma intersecção completa com singularidade isolada (ICIS) $X$ admite uma deformação versal $\mathfrak{X}$ cuja fibra genérica $X_{t}$ é suave e tem o tipo de homotopia de um bouquet de esferas [29]. O número de esferas do bouquet é chamado de número de Milnor da ICIS.

Lê D. T. [50] e Greuel [25] estabeleceram uma fórmula indutiva que permite calcular o número de Milnor de uma ICIS. Sejam $f=\left(f_{1}, \ldots, f_{k-1}, f_{k}\right):\left(\mathbb{C}^{r}, 0\right) \longrightarrow\left(\mathbb{C}^{k}, 0\right)$ e $g=$ $\left(f_{1}, \ldots, f_{k-1}\right):\left(\mathbb{C}^{r}, 0\right) \longrightarrow\left(\mathbb{C}^{k-1}, 0\right)$. Indiquemos por $X=g^{-1}(0)$ e $Y=f^{-1}(0)=X \cap f_{k}^{-1}(0)$. Denotemos por $J\left(f_{1}, \ldots, f_{k}\right)$ o ideal de todos os menores $k \times k$ da matriz jacobiana de $f$. Então,

$$
\mu(X, 0)+\mu(Y, 0)=\operatorname{dim}_{\mathbb{C}} \frac{\mathcal{O}_{r}}{\left\langle f_{1}, \ldots, f_{k-1}, J\left(f_{1}, \ldots, f_{k}\right)\right\rangle} .
$$

Por outro lado,

$$
\operatorname{dim}_{\mathbb{C}} \frac{\mathcal{O}_{r}}{\left\langle f_{1}, \ldots, f_{k-1}, J\left(f_{1}, \ldots, f_{k}\right)\right\rangle}
$$

é igual ao número de pontos críticos, contando a multiplicidade, de $f_{k}$ restrita à fibra de $g$.

Nesta seção, consideramos um problema análogo para as variedades determinantais que são Cohen-Macaulay de codimensão dois e que têm singularidade isolada na origem. Nesta classe, estão as superfícies determinantais em $\mathbb{C}^{4}$ e as 3 -variedades em $\mathbb{C}^{5}$. Para estas variedades, se verificam a existência e unicidade da suavização, o que possibilita a seguinte definição:

Definição 6.1.1. Sejam $(X, 0) \subset\left(\mathbb{C}^{r}, 0\right)$, um germe de variedade determinantal de codimensão 2, com singularidade isolada na origem de dimensão $d=2,3$. Definimos o número de Milnor de $X$ como sendo $\mu(X)=b_{d}\left(X_{t}\right)$, onde $X_{t}$ é a fibra genérica de $X$ e $b_{d}\left(X_{t}\right)$ é o d-ésimo número de Betti de $X_{t}$.

Seja $p: X \longrightarrow \mathbb{C}$ uma função analítica complexa com singularidade isolada na origem. Então, $Y=X \cap p^{-1}(0)$ é uma variedade de dimensão $d-1$, com singularidade isolada em 0 .

Em particular, quando $p: \mathbb{C}^{r} \longrightarrow \mathbb{C}$ é uma função linear, temos a partir da matriz de representação que $Y$ é também uma variedade determinantal de dimensão $d-1 \mathrm{em} \mathbb{C}^{r-1}$.

No teorema a seguir, obtemos uma fórmula do tipo Lê- Greuel para germes de superfícies determinantais, Cohen-Macaulay de codimensão 2 com singularidade isolada na origem.

Teorema 6.1.1. Seja $(X, 0) \subset\left(\mathbb{C}^{4}, 0\right)$ o germe de uma superfície determinantal com singularidade isolada definida como acima. Seja $p:\left(\mathbb{C}^{4}, 0\right) \longrightarrow(\mathbb{C}, 0)$ uma função linear cuja restrição $\grave{a} X$ tem singularidade isolada na origem. Então,

$$
m_{2}(X, p)=\mu(X, p)+\mu(X),
$$


onde $\mu(X, p)$ é o número de Milnor da curva determinantal $Y=X \cap p^{-1}(0)$ e $m_{2}(X, p)$ é a multiplicidade polar de $X$ relativa à $p$.

\section{Demonstração.}

Denotemos por $\mathfrak{X}$ o espaço total de uma suavização de $X$ cuja fibra genérica denotamos por $X_{t}$ e $\widetilde{p}: \mathfrak{X} \longrightarrow \mathbb{C}$ uma função analítica complexa tal que $\widetilde{p}(x, 0)=p(x)$ e para todo $t \neq 0$, $\widetilde{p}(\cdot, t)=p_{t}$ é uma função de Morse em $X_{t}$. Usando a decomposição 5.4, temos

$$
\mathcal{X}\left(X_{t}\right)=\mathcal{X}\left(\left(p_{t}\right)^{-1}(0)\right)+(-1)^{2} n_{\sigma},
$$

onde $n_{\sigma}$ é o número de pontos críticos de $p_{t}$. Além disso,

$$
\mathcal{X}\left(X_{t}\right)=b_{0}\left(X_{t}\right)-b_{1}\left(X_{t}\right)+b_{2}\left(X_{t}\right)
$$

Logo, usando (5.4) obtemos

$$
b_{0}\left(X_{t}\right)-b_{1}\left(X_{t}\right)+b_{2}\left(X_{t}\right)=\mathcal{X}\left(\left(p_{t}\right)^{-1}(0)\right)+n_{\sigma} .
$$

Observemos que $p_{t}^{-1}(0) \cap X_{t}$ é a fibra genérica da variedade determinantal $p^{-1}(0) \cap X$.

Como $X$ é uma superfície Cohen-Macaulay de codimensão 2, sabemos que $p_{t}^{-1}(0) \cap X_{t}$ é a fibra genérica da curva determinantal $p^{-1}(0) \cap X$. Portanto, $p_{t}^{-1}(0)$ tem o tipo de homotopia de um bouquet de esferas de dimensão real 1. Sejam $C=p^{-1}(0) \cap X$ e $C_{t}=p_{t}^{-1}(0) \cap X_{t}$ a fibra genérica de $C$. Então, $C$ é uma curva reduzida determinantal e $\mathcal{X}\left(C_{t}\right)=1-\mu(C, 0)$, onde $\mu(C, 0)$ é o número de Milnor da curva.

Notemos que $n_{\sigma}$ coincide com a $d$-ésima multiplicidade polar da variedade $P_{d}(f, p)$, isto é, $n_{\sigma}=m_{2}(X, p)$. Como variedades determinantais são normais, sabemos que $b_{1}\left(X_{t}\right)=0$. Além disso $X_{t}$ é conexa. Portanto,

$$
1+b_{2}\left(X_{t}\right)=1-\mu\left(p^{-1}(0) \cap X\right)+m_{2}(X, p) .
$$

Logo

$$
m_{2}(X, p)=\mu\left(p^{-1}(0) \cap X\right)+\mu(X) .
$$

Quando $p: \mathbb{C}^{r}, 0 \longrightarrow \mathbb{C}$, 0 é uma função linear genérica, $\mu(X, p)=\mu^{(1)}(X)$ é o número de Milnor de uma seção genérica de $X$ e $m_{2}(X, p)=m_{2}(X)$ a segunda multiplicidade polar de $X$.

Neste caso, a fórmula acima relaciona invariantes da variedade $X$. Temos, portanto o corolário: 
Corolário 6.1.1. Seja $(X, 0) \subset\left(\mathbb{C}^{4}, 0\right)$ o germe de uma superfície determinantal com singularidade isolada e $p: X \longrightarrow(\mathbb{C}, 0)$ uma função linear genérica com singularidade isolada em $X$. Então,

$$
m_{2}(X)=\mu^{(1)}(X)+\mu(X)
$$

onde $\mu^{(1)}(X)$ é o número de Milnor da curva determinantal $Y=X \cap p^{-1}(0)$ e $m_{2}(X)$ é a multiplicidade polar de $X$.

Podemos provar, de maneira análoga ao que foi feito para superfícies determinantais, resultados semelhantes para 3 - variedades de codimensão $2 \mathrm{em}\left(\mathbb{C}^{5}, 0\right)$ com singularidade isolada na origem. Entretanto, não obtemos uma fórmula do tipo de Lê- Greuel porque não sabemos se o segundo número de Betti se anula ou não na fibra genérica.

Proposição 6.1.1. Sejam $(X, 0) \subset\left(\mathbb{C}^{5}, 0\right)$ o germe de uma variedade determinantal de codimensão 2 com singularidade isolada na origem e $\mathfrak{X}$ o espaço total de uma suavização de $X$ cuja fibra genérica, denotamos por $X_{t}$. Se $p:\left(\mathbb{C}^{5}, 0\right) \longrightarrow(\mathbb{C}, 0)$ é uma função linear cuja restrição a $X$ tem singularidade isolada na origem, então

a)

$$
m_{3}(X, p)=\mu\left(p^{-1}(0) \cap X, p\right)+\mu(X)-b_{2}\left(X_{t}\right),
$$

onde $m_{3}(X, p)$ é a multiplicidade polar de $X$ relativa à $p$.

b) Se p é uma função linear genérica, então

$$
m_{3}(X)=\mu^{(1)}(X)+\mu(X)-b_{2}\left(X_{t}\right)
$$

onde $m_{3}(X)$ é a multiplicidade polar de $X$.

\section{2 Índice de 1-Formas em Variedades Determinantais}

Em [13], W. Ebeling e S. M. Gusein- Zade definem índices de 1- formas em variedades determinantais (que têm singularidade essencialmente isoladas, EIDS). Estes índices são extensões do índice de Poincaré- Hopf e podem ser calculados usando diferentes resoluções de tais variedades. Para variedades determinantais $X$ com singularidade isolada todos os índices definidos em [13] 
coincidem. Vamos aqui apresentar somente a definição que usa a transformada de Nash, $\widehat{\Delta_{t}}$, da variedade $\Delta_{t} \subset \operatorname{Mat}_{(p, m)}(\mathbb{C})$.

Iniciamos com a definição de suavização essencial. Seja $(X, 0) \subset\left(\mathbb{C}^{r}, 0\right)$ uma variedade determinantal com singularidade isolada e $F:\left(\mathbb{C}^{r}, 0\right) \longrightarrow\left(\operatorname{Mat}_{(n, p)}(\mathbb{C}), 0\right)$ tal que $X=F^{-1}\left(\Delta_{t}\right)$ e para todo $i \leq t, F$ é transversal a $\Delta_{i} \backslash \Delta_{i-1}$ em todos os pontos $x$ de uma vizinhança da origem em $\mathbb{C}^{r}$.

Definição 6.2.1. Uma suavização essencial $\mathcal{X}$ de $(X, 0)$ é uma subvariedade de uma vizinhança $U$ da origem em $\mathbb{C}^{r}$ definida por uma perturbação $\widetilde{F}: U \longrightarrow \operatorname{Mat}_{(n, p)}(\mathbb{C})$ do germe $F$, transversal a todos os estratos $\Delta_{i} \backslash \Delta_{i-1}$ para todo $i \leq t$.

Pelo Teorema de Transversalidade de Thom, uma perturbação genérica define uma suavização essencial de $(X, 0)$. Em termos da teoria de singularidades de matrizes que desenvolvemos anteriormente, (ver 2.4.1), uma suavização essencial ocorre quando $F$ admite uma estabilização com singularidade isolada. Além disso, uma suavização essencial é uma suavização no sentido usual se, e somente se, $r<(n-t+2)(p-t+2)$.

Dada uma variedade $Z \subset U \subset \mathbb{C}^{r}$ analítica complexa qualquer de dimensão pura $d$, definimos a transformada de Nash por

$$
\widehat{Z}=\overline{\left\{(x, W) \mid x \in Z_{r e g}, W=T_{x} Z_{r e g}\right\}}
$$

onde $Z_{\text {reg }}$ é a parte regular de $Z$. Aqui, o fecho é tomado em $Z \times G r\left(d, \mathbb{C}^{r}\right)$, onde $G r\left(d, \mathbb{C}^{r}\right)$ é a Grassmanniana de $d$ planos em $\mathbb{C}^{r}$. A transformada de Nash é, em muitos casos, singular.

Para construir a transformada de Nash para a variedade $\Delta_{t} \operatorname{denotamos}$ por $d=\operatorname{dim}\left(\Delta^{t}\right)=$ $n p-(n-t+1)(p-t+1)$ e $\operatorname{Gr}\left(d, \operatorname{Mat}_{(n, p)}(\mathbb{C})\right)$ a variedade Grassmanniana de subespaços de dimensão $d$ de $\operatorname{Mat}_{(n, p)}(\mathbb{C})$.

Seja $A \in \operatorname{Mat}_{(n, p)}(\mathbb{C})$ uma matriz de posto $t-1$. Podemos considerar a matriz $A$ como um operador $\mathbb{C}^{n} \longrightarrow \mathbb{C}^{p}$. O espaço tangente a $\Delta_{t}$ no ponto $A$ é dado por

$$
T_{A} \Delta_{t}=\left\{B \in \operatorname{Mat}_{(n, p)}(\mathbb{C}) \mid B(k e r(A)) \subset \operatorname{Im}(A)\right\} .
$$

Consideremos a aplicação

$$
\begin{aligned}
\alpha: G r(n-t+1, n) \times G r\left(t-1, \mathbb{C}^{p}\right) & \left.\longrightarrow \operatorname{Gr}_{\left(d, \operatorname{Mat}_{(n,}, \mathbb{C}^{p}\right)}(\mathbb{C})\right) \\
\left(W_{1}, W_{2}\right) & \longmapsto B
\end{aligned}
$$

onde $B \in \operatorname{Mat}_{(n, p)}(\mathbb{C})$ é tal que $B\left(W_{1}\right) \subset W_{2}$. 
Observemos que o subespaço $\left\{B \in \operatorname{Mat}_{(n, p)}(\mathbb{C}) \mid B\left(W_{1}\right) \subset W_{2}\right\}$ tem dimensão $d$ e a aplicação $\alpha$ é um mergulho. O fecho de

$$
\left\{A \in \operatorname{Mat}_{(n, p)} \mid \operatorname{ker}(A)=W_{1}, \operatorname{Im}(A)=W_{2}\right\}
$$

consiste de todos os operadores $A$ com $\operatorname{Ker}(A) \supset W_{1}$ e $\operatorname{Im}(A) \subset W_{2}$.

Portanto, a transformada de Nash de $\Delta_{t}$ é dada por

$$
\widehat{\Delta_{t}}=\left\{(A, W) \mid W=\alpha\left(W_{1}, W_{2}\right), \operatorname{Ker}(A) \supset W_{1}, \operatorname{Im}(A) \subset W_{2}\right\} .
$$

Isto significa que a projeção $\widehat{\Delta_{t}}$ no segundo fator é a projeção de um fibrado vetorial de posto $(t-1)^{2}$ sobre $\alpha\left(G r\left(n-t+1, \mathbb{C}^{n}\right) \times G r\left(t-1, \mathbb{C}^{p}\right)\right)$. Assim, a transformada de Nash dá uma resolução $\pi: \widehat{\Delta_{t}} \longrightarrow \Delta_{t}$ da variedade $\Delta_{t}$.

Sejam $(X, 0)$ uma variedade determinantal com singularidade isolada e $\mathcal{X}$ uma suavização essencial de $(X, 0)$. Se $\bar{X}=\Delta_{t} \times \mathcal{X}$ então a aplicação $\Pi: \bar{X} \longrightarrow \mathcal{X}$ é uma resolução da variedade $\mathcal{X}$.

Tomemos $\omega$ o germe de uma 1 -forma analítica complexa em $\left(\mathbb{C}^{r}, 0\right)$ cuja restrição a $(X, 0)$ tem ponto singular isolado na origem. A 1- forma $\omega$ define uma seção não nula $\widehat{\omega}$ no fibrado de Nash dual $\widehat{\mathbb{T}}^{*}$ sobre a pre- imagem de $\mathcal{X} \cap \mathcal{S}_{\epsilon}$.

Definição 6.2.2. O indice de Poincaré- Hopf (PH-indice), $\operatorname{ind}_{P H N} \omega=\operatorname{ind}_{P H}(\omega ; X, 0)$ da 1 forma $\omega$ numa $\operatorname{EIDS}(X, 0)$ é a obstrução para estender a seção não nula $\widehat{\omega}$ do dual do fibrado de Nash $\widehat{\mathbb{T}}^{*}$ da pre-imagem da fronteira $S_{\epsilon}=\partial B_{\epsilon}$ para a pré-imagem de seu interior, isto é, para a variedade $\widehat{\Delta_{t}} \times \mathcal{X}$.

Proposição 6.2.1. ([13], pág. 7) O PH-índice, ind ${ }_{P H} \omega$ da 1-forma $\omega$ numa EIDS $(X, 0)$ é igual ao número de pontos singulares não-degenerados de uma deformação genérica $\widetilde{\omega}$ de uma 1-forma $\omega$ sobre $\mathcal{X}_{\text {reg }}$, que é a parte regular da variedade $\mathcal{X}$.

Segue desta proposição que o PH- índice está intimamente relacionado com a $d$-ésima multiplicidade polar.

É possível também relacionar o $i_{n d} d_{P H}$ com a noção de índice radial estudada em [14] por W. Ebeling e S. M. Guzein- Zade. Sejam $(X, 0) \subset\left(\mathbb{C}^{r}, 0\right)$ o germe de uma variedade complexa analítica de dimensão pura $n$ e $\omega$ uma 1-forma complexa (genericamente contínua) numa vizinhança da origem de $\mathbb{C}^{r}$.

Definição 6.2.3. O indice radial da 1-forma $\omega$ em $X$ na origem, ind rad $_{\text {rad }}(\omega, 0)$, é dado por

$$
i n d_{\text {rad }}(\omega ; X, 0)=(-1)^{n} i n d_{r a d}(\operatorname{Re}(\omega) ; X, 0),
$$

onde $\operatorname{Re}(\omega)$ denota a parte real da 1- forma $\omega$. 
Sejam $f:(X, 0) \longrightarrow(\mathbb{C}, 0)$ o germe de uma função analítica complexa, com singularidade isolada na origem e $\omega$ a diferencial de $f$, então podemos relacionar o índice radial com a característica de Euler da fibra Milnor de $f$ da seguinte maneira:

Teorema 6.2.1. ([14], pág. 237) Sejam $f:(X, 0) \longrightarrow(\mathbb{C}, 0)$ o germe de uma função analítica complexa com singularidade isolada na origem, então

$$
\operatorname{ind}_{\text {rad }}(d f ; X, 0)=(-1)^{n} \overline{\mathcal{X}}\left(f^{-1}(t)\right)
$$

onde $t \in \mathbb{C}, t \neq 0$ e $\overline{\mathcal{X}}\left(f^{-1}(t)\right)=\mathcal{X}\left(f^{-1}(t)\right)-1$.

Para variedades determinantais com singularidade isolada, a relação entre PH-índice e o índice radial, (ver [14]), é dada por

$$
\operatorname{ind}_{P H}(\omega ; X, 0)=\operatorname{ind}_{\text {rad }}(\omega ; X, 0)+(-1)^{\operatorname{dim}(X)} \overline{\mathcal{X}}(X, 0),
$$

onde $\overline{\mathcal{X}}(X, 0)=\mathcal{X}(X, 0)-1$.

Na próxima proposição, relacionamos os resultados de W. Ebeling e S. M. Gusein-Zade e os resultados obtidos na seção anterior, no caso em que $\omega=d f$, onde $f$ é uma projeção linear genérica.

Proposição 6.2.2. Sejam $(X, 0) \subset \mathbb{C}^{4}$ uma superfície determinantal, Cohen- Macaulay, com singularidade isolada na origem. Então, $\operatorname{ind}_{P H}(\omega ; X, 0)=m_{2}(X)$, onde $\omega=d p$ para p projeção genérica e $m_{2}(X)$ é a segunda variedade polar de $X$.

\section{Demonstração.}

Sejam $p:(X, 0) \longrightarrow \mathbb{C}$ uma projeção linear genérica e $\epsilon>0$ pequeno o suficiente tal que a restrição de $p$ a $X \cap B_{\epsilon}(0)$ tenha ponto crítico isolado na origem. Então, pelo Teorema 3 de $[14]$

$$
\operatorname{ind}_{\text {rad }}(d p ; X, 0)=(-1)^{d} \overline{\mathcal{X}}\left(p^{-1}(t)\right)
$$

onde $t \neq 0$ e $\overline{\mathcal{X}(X)}=\mathcal{X}(X)-1$. Assim,

$$
\operatorname{ind}_{P H}(d p ; X, 0)=(-1)^{2} \overline{\mathcal{X}}\left(p^{-1}(t)\right)+(-1)^{2} \overline{\mathcal{X}}(X, 0) .
$$

Logo,

$$
\operatorname{ind}_{P H}(d p ; X, 0)=\left(\mathcal{X}\left(p^{-1}(t)\right)-1\right)+(\mathcal{X}(X, 0)-1)=\mu(C)+\mu(X),
$$

onde $C=p^{-1}(0) \cap X$.

Usando o Teorema 6.1.1 e resultado anterior, temos: 
Corolário 6.2.1. Sejam $(X, 0) \subset \mathbb{C}^{4}$ uma superfície determinantal, Cohen- Macaulay, com singularidade isolada na origem, $p:(X, 0) \longrightarrow \mathbb{C}$ uma projeção linear genérica e $\omega=d p$. Então,

$$
\mu(X)+\mu(C)=\operatorname{ind}_{P H}(\omega ; X, 0)
$$

onde $C=p^{-1}(0) \cap X$.

Observação 6.2.1. a) Este resultado é útil nos cálculos para determinar $\mu(X)$, uma vez que em muitos casos é possivel utilizar métodos geométricos para calcular ind ${ }_{P H}(\omega ; X, 0)$. Este procedimento será usado nos cálculos dos exemplos da próxima seção.

b) Um problema importante que não abordamos neste trabalho é a determinação de uma fórmula algébrica semelhante à que aparece na fórmula de Lê- Greuel para ICIS que facilita o cálculo do número de Milnor. Em [21], T. Gaffney e N. Grulha Jr. determinam uma relação entre indices de famílias de formas e a multiplicidade de pares de módulos.

\subsection{Exemplos}

Nesta seção, verificamos que o número de Tjurina $\tau(X)$ e o número de Milnor $\mu(X)$ satisfazem a relação $\tau(X)=\mu(X)+1$, para algumas superfícies determinantais simples $X \subset \mathbb{C}^{4}$.

Relações entre estes dois invariantes são conhecidas para diferentes classes de singularidades. Um resultado importante obtido por K. Saito em 1971, estabelece que uma hipersuperfície com singularidade isolada é quase-homogênea se, e somente se, o número de Milnor é igual ao número de Tjurina ([44]). O resultado de K. Saito foi estendido para intersecções completas com singularidade isolada de dimensão 1, por G. M. Greuel, B. Martin e G. Pfister [26]. Utilizando técnicas diferentes J. Wahl prova resultado análogo para ICIS de dimensão 2 em [54].

Para a classe de variedades determinantais, D. Mond e V. Goryunov mostram que a relação $\mu(X)=\tau(X)$ se verifica para germe de matrizes quase- homogêneas simétricas de qualquer ordem, para matrizes quadradas arbitrárias dependendo de até três parâmetros e, para matrizes anti- simétricas de ordem $2 n \times 2 n$ em 5 variáveis, $n \in \mathbb{N}$. Neste artigo, o número de Tjurina $\tau$, é a dimensão do espaço das deformações de primeira ordem de uma matriz $M \in M a t_{m}(\mathcal{P})$ e $\mu$ é o número de Milnor da variedade $X=\operatorname{det}^{-1}(0)$, onde det denota a função determinante da matriz $M$. 
Para curvas determinantais simples, classificadas por A. Frühbis-Krüger a relação $\tau(X)=$ $\mu(X)+1$ pode ser observada nas tabelas em [16].

Seja $M$ um germe simples de matriz que define uma superfície $X \subset \mathbb{C}^{4}$ e $\omega$ uma forma linear genérica em $X$. Para verificar a relação $\tau(X)=\mu(X)+1$ para os germes simples de superfícies em $\mathbb{C}^{4}$, é suficiente determinar $\tau(X)$ e $\mu(X)$ para cada forma normal da lista de [18]. O número de Tjurina é a $\mathcal{G}_{e^{-}}$codimensão de $M$ e, pode ser encontrado em [18], pág. 9. Para calcular o número de Milnor, utilizamos a fórmula $m_{2}(X)=\mu^{(1)}(X)+\mu(X)$, obtida no Corolário 6.1.1, onde $\mu^{(1)}(X)$ é o número de Milnor da curva $X \cap p^{-1}(0)$, onde $p: X \longrightarrow \mathbb{C}$ é uma projeção linear genérica.

Usando o corolário 6.2.1, sabemos que $m_{2}(X)=i n d_{P H}(\omega ; X, 0)$. Por outro lado, se $X$ é simples e $p$ é uma projeção linear genérica, $Y=X \cap p^{-1}(0)$ é uma curva determinantal simples seu número de Milnor pode ser calculado ou podemos usar diretamente a tabela de curvas simples em [16], pág. 4008- 4009.

Para encontrar $m_{2}(X)$, ou equivalentemente, $\operatorname{ind}_{P H}(\omega ; X, 0)$, podemos utilizar em cada caso um dos seguintes procedimentos:

a) Usamos o algoritmo proposto por W. Ebeling e S. M. Gusein-Zade em [13] para calcular o ind $_{P H} \omega$ através das seguintes etapas:

- Obter uma curva $Y=X \cap p^{-1}(0)$, tal que $Y$ é uma curva determinantal reduzida e $p: \mathbb{C}^{4} \longrightarrow \mathbb{C}$ é uma projeção linear genérica.

- Calcular o desdobramento versal $\mathcal{N}$ da matriz $N, 2 \times 3$, que define $Y$ e obter em $\mathcal{N}$ uma suavização $X_{\lambda}$ de $X$. Para cada $\lambda$ fixo a superfície $X_{\lambda}$ é uma deformação a um parâmetro da curva $Y$.

- Contar o número de pontos para os quais a família a um parâmetro intercepta o discriminante de $\mathcal{N}$.

b) Calculamos o número de pontos singulares não degenerados da forma linear $\omega$ definida em uma suavização de $X$.

c) Obtemos uma perturbação $X_{t}$ de $X$ com pontos singulares $p_{1}, \ldots, p_{l}$ e usamos o fato que

$$
\mu(X)=\sum_{i=1}^{l} \mu\left(X_{t}, p_{i}\right)
$$

A seguinte tabela, apresenta as formas normais dos germes simples de superfícies determinantais classificadas por A. Frühbis-Krüger, A. Neumer em [18]. O número de Tjurina, $\tau(X)$ foi calculado em [18]. Nossa contribuição é o cálculo do número de Milnor. 


\begin{tabular}{|c|c|c|c|c|c|}
\hline Tipo do jato & Matriz de Representação & & $\tau$ & $\mu$ & $\begin{array}{c}\text { Nome do ponto } \\
\text { triplo em [46] }\end{array}$ \\
\hline$J^{(4,1)}$ & $\left.\begin{array}{ccc}w & y & x \\
z & w & y\end{array}\right)$ & & 2 & 1 & $A_{0,0,0}$ \\
\hline$J^{(4,2)}$ & $\left(\begin{array}{ccc}w & y & x \\
z & w & y^{k}\end{array}\right)$ & $k \geq 2$ & $k+1$ & $k$ & $A_{0,0, k-1}$ \\
\hline$J^{(4,3)}$ & $\left(\begin{array}{ccc}w^{l} & y & x \\
z & w & y^{k}\end{array}\right)$ & $k \geq l \geq 2$ & $k+l$ & $k+l-1$ & $A_{0, l-1, k-1}$ \\
\hline \multirow[t]{4}{*}{$J^{(4,4)}$} & $\left(\begin{array}{ccc}z & y & x \\
x & w & y^{2}+z^{k}\end{array}\right)$ & $k \geq 2$ & $k+3$ & $k+2$ & $C_{k+1,0}$ \\
\hline & $\begin{array}{l}\left(\begin{array}{ccc}z & y & x \\
x & w & y z+y^{k} w\end{array}\right) \\
\left(\begin{array}{ccc}z & y & x \\
x & w & y z+y^{k}\end{array}\right)\end{array}$ & $\begin{array}{l}k \geq 1 \\
k \geq 3\end{array}$ & $\begin{array}{l}2 k+4 \\
2 k+1\end{array}$ & $2 k+3$ & $\begin{array}{l}B_{2 k+2,0} \\
B_{2 k-1,0}\end{array}$ \\
\hline & $\left(\begin{array}{ccc}z & y & x \\
x & w & z^{2}+y w\end{array}\right)$ & & 7 & 6 & $D_{0}$ \\
\hline & $\left(\begin{array}{ccc}z & y & x \\
x & w & z^{2}+y^{3}\end{array}\right)$ & & 8 & 7 & $F_{0}$ \\
\hline$J^{(3,1)}$ & $\left(\begin{array}{ccc}z & y+w^{l} & w^{m} \\
w^{k} & y & x\end{array}\right.$ & $k, l, m \geq 2$ & $k+l+m-1$ & & $A_{k-1, l-1, m-1}$ \\
\hline \multirow[t]{4}{*}{$J^{(3,2)}$} & $\left(\begin{array}{ccc}z & y & x^{l}+w^{2} \\
w^{k} & x & y\end{array}\right)$ & $k, l \geq 2$ & $k+l+2$ & $k+l+1$ & $C_{l+1, k-1}$ \\
\hline & $\left(\begin{array}{ccc}z & y+w^{l} & x w \\
w^{k} & x & y \\
z & y & x w+w^{l} \\
w^{k} & x & y\end{array}\right)$ & $\begin{array}{c}k, l \geq 2 \\
k \geq 2, l \geq 3\end{array}$ & $\begin{array}{c}k+2 l+1 \\
k+2 l\end{array}$ & & $\begin{array}{c}B_{2 l, k-1} \\
B_{2 l+1, k-1}\end{array}$ \\
\hline & $\left(\begin{array}{ccc}z & y+w^{2} & x^{2} \\
w^{k} & x & y\end{array}\right)$ & $k \geq 2$ & $k+6$ & $k+5$ & $D_{k-1}$ \\
\hline & $\left(\begin{array}{ccc}z & y & x^{2}+w^{3} \\
w^{k} & x & y\end{array}\right.$ & $k \geq 2$ & $k+7$ & $k+6$ & $F_{k-1}$ \\
\hline$J(3,3)$ & $\begin{array}{l}\left(\begin{array}{ccc}z & y & x w+w^{k} \\
y & x & z\end{array}\right) \\
\left(\begin{array}{ccc}z & y & x w \\
y & x & z+w^{k}\end{array}\right) \\
\left(\begin{array}{ccc}z & y & x w \\
y+w^{k} & x & z\end{array}\right)\end{array}$ & & $\begin{array}{l}3 k+1 \\
3 k+2 \\
3 k+3\end{array}$ & & $\begin{array}{c}H_{3 k} \\
H_{3 k+1} \\
H_{3 k+2}\end{array}$ \\
\hline
\end{tabular}




\begin{tabular}{|l|l|l|l|l|l|}
\hline & $\left(\begin{array}{ccc}z & y & w^{2} \\
y & x & z+x^{2}\end{array}\right)$ & 8 & & \\
\hline$\left(\begin{array}{ccc}z & y & x^{3}+w^{2} \\
y & x & z\end{array}\right)$ & & 9 & & \\
\hline$\left(\begin{array}{ccc}z & y & x^{2} \\
y & x & z+w^{2}\end{array}\right)$ & & 9 & & \\
\hline
\end{tabular}

Tabela 6.1: Formas normais de superfícies simples em $\mathbb{C}^{4}$

Para facilitar o entendimento dos cálculo acrescentamos também a primeira parte da tabela com as formas normais das curvas determinantais simples obtida em ([16]).

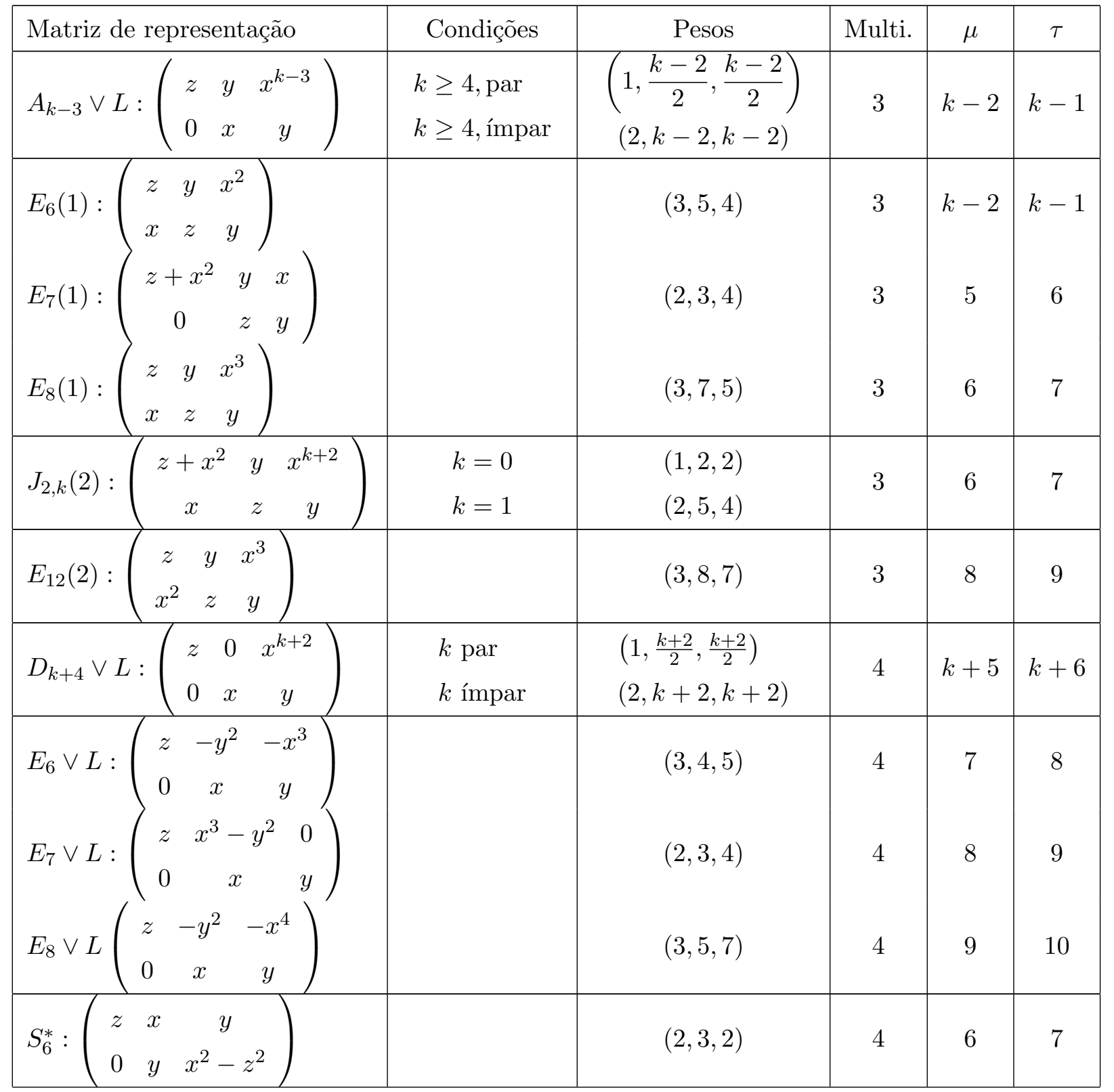




\begin{tabular}{|l|l|l|l|l|l|}
\hline$T_{7}^{*}:\left(\begin{array}{ccc}z & x & y \\
0 & y & x^{2}-z^{3}\end{array}\right)$ & $(6,9,4)$ & 4 & 7 & 8 \\
\hline$U_{7}^{*}:\left(\begin{array}{ccc}z & x y & x^{2} \\
x & z & y\end{array}\right)$ & & $(3,4,5)$ & 4 & 7 & 8 \\
\hline$W_{8}^{*}:\left(\begin{array}{ccc}z & y^{2} & x^{2} \\
x & z & y\end{array}\right)$ & & $(4,5,7)$ & 4 & 8 & 9 \\
\hline
\end{tabular}

Tabela 6.2: Formas Normais de curvas simples em $\mathbb{C}^{3}$

A seguir apresentamos um resumos dos cálculos feitos para calcular o número de Milnor de algumas formas normais de superfícies determinantais.

\section{- $1^{\text {o }}$ Forma Normal:}

Sejam $p: \mathbb{C}^{4} \longrightarrow \mathbb{C}$ e $\omega=d p$. Consideremos a curva espacial $(C, 0)$ determinada pela matriz

$$
N=\left(\begin{array}{lll}
z & y & x \\
0 & x & y
\end{array}\right) .
$$

O desdobramento versal de $(C, 0)$ é dado por

$$
\left(\begin{array}{ccc}
z & y+b & x+c \\
a & x & y
\end{array}\right),
$$

cujo discriminante é $a\left(b^{2}-c^{2}\right)=0$.

Podemos obter $M$ a partir da deformação versal de $N$ tomando $a=w, b=c=0$ e, além disso, uma suavização para $M$ é obtida tomando $a=b=w, c=\lambda \neq 0$. Para cada $\lambda$ fixo, a correspondente família a 1- parâmetro em $w$ intercepta o discriminante em 3 pontos distintos, onde a função em $p(x, y, z, w)=w$ tem pontos críticos não- degenerados. Usando 6.1.1, obtemos

$$
3=\mu(X)+\mu(C),
$$

como $\mu(C)=2$, segue que $\mu(X)=1$.

\section{- $2^{\text {o }}$ Forma Normal:}

Para a segunda forma normal, sejam $p: X \longrightarrow \mathbb{C}$ a projeção linear genérica, dada por $p(x, y, z, w)=w, \omega=d p$ e $(C, 0)$ a curva determinantal dada por

$$
\left(\begin{array}{lll}
z & x & y^{k} \\
0 & y & x
\end{array}\right), k \geq 1 .
$$


que tem desdobramento versal dado por

$$
\left(\begin{array}{ccc}
z & x+b & y^{k}+\sum_{i=0}^{k-1} c_{i} x^{i} \\
a & y & x
\end{array}\right) .
$$

Uma suavização da superfície determinantal $X$ é obtida tomando $c_{0}=\lambda \neq 0, a=b=w$ e $c_{i}=0$ para $i \neq 0$. Denotemos por $M_{\lambda}$ a matriz obtida desta forma.

Para cada $\lambda$ fixo, sejam $f_{\lambda}: \mathbb{C}^{4} \longrightarrow \mathbb{C}^{3}$ a aplicação determinada pelos menores $2 \times 2$ de $M_{\lambda}, J f_{\lambda}$ a matriz jacobiana de $f_{\lambda}$ e, por $\left[J f_{\lambda}, \omega\right]$ a matriz $4 \times 4$ cujas três primeiras linhas são as linhas de $J f_{\lambda}$ e a última linha são os coeficientes da forma $\omega$.

Para determinar o número de pontos críticos não- degenerados de $\omega$ na superfície determinada por $M_{\lambda}$, precisamos determinar o número de soluções das equações

$$
\begin{aligned}
& z x-y w-w^{2}=0 \\
& z y-w x^{k}-\lambda w=0 \\
& y^{2}+y w-x^{k+1}-\lambda x=0 \\
& z^{2}-k x^{k 1} w^{2}=0 \\
& z y+k x^{k} w=0 \\
& w y+z x=0 \\
& -k x^{k-1} w(2 y+w)+z\left((k+1) x^{k}+\lambda\right)=0 \\
& (k+1) x^{k} y+\lambda y=0 \\
& 2 y^{2}+y w=0 \\
& z(2 y+w)-w\left((k+1) x^{k}+\lambda\right)=0 \\
& (k+1) x^{k+1}+\lambda x=0 \\
& 2 y x+x w=0
\end{aligned}
$$

as três primeiras equações acima são determinadas pelos menores $2 \times 2$ da matriz $M_{\lambda}$, as outras são obtidas pelos menores $3 \times 3$ de $\left[J f_{\lambda}, \omega\right]$. Neste caso, temos $2 k$ soluções da forma $\left(x,\left(k x^{k+1}\right)^{\frac{1}{2}}, 2 k x^{k},-2\left(k x^{k+1}\right)^{\frac{1}{2}}\right)$ e $\lambda=(k+1) x^{k}$. É fácil verificar que estas singularidades correspondem a pontos singulares da primeira forma normal de curvas.

Portanto, $\operatorname{ind}_{P H}(\omega)=2 k$.

Logo,

$$
\mu(X)=2 k-(k+1)=k .
$$

\section{- $\underline{3^{\circ} \text { e } 4^{\circ} \text { Formas Normais: }}$}


Sejam $p_{i}: \mathbb{C}^{4} \longrightarrow \mathbb{C}, i=1,2$, projeções lineares dadas por $p_{1}(x, y, z, w)=w-x$ e $p_{2}(x, y, z, w)=y$ para a terceira e quarta formas normais, respectivamente.

Em ambos os casos, a curva determinantal simples $C=X \cap p^{-1}(0)$, é definida pela matriz

$$
\left(\begin{array}{ccc}
z & x & y^{k} \\
0 & y & x
\end{array}\right), k \geq 1 .
$$

que é a primeira forma normal de curvas simples encontrada em [16]. Procedendo de maneira análoga ao que foi feito para a segunda forma normal, podemos mostrar que nestes casos, $\tau(X)=\mu(X)+1$.

- $5^{\mathbf{o}}$ Forma Normal: Para determinar o número de Milnor desta forma normal usaremos o terceiro procedimento e indução sobre $k$. Denotamos por $M$ a matriz de representação da $5^{\circ}-(a)$ forma normal. Consideremos, inicialmente, o caso $k=1$, isto é

$$
M=\left(\begin{array}{ccc}
z & y & x \\
x & w & y z+y w
\end{array}\right) .
$$

Sejam $p: \mathbb{C}^{4} \longrightarrow \mathbb{C}$ dada por $p(x, y, z, w)=y-z$ e $\omega=d p$. Neste caso, a curva determinantal dada por $C=X \cap p^{-1}(0)$ é definida pela matriz

$$
\left(\begin{array}{lll}
w & x & z^{2} \\
0 & z & x
\end{array}\right)
$$

que é também a primeira forma normal de [16] $\operatorname{com} \mu(C)=3$. Uma suavização de $X$ é obtida por

$$
\left(\begin{array}{ccc}
w & x+\lambda & t z^{2}+y z+y w \\
y & z & x
\end{array}\right)
$$

onde $t \in \mathbb{C}$, e $t \neq 0$.

Precisamos determinar o número de soluções comuns das equações

$$
\begin{aligned}
& w z-y(x+\lambda)=0 \\
& w x-y\left(t z^{2}+y z+y w\right)=0 \\
& x(x+\lambda)-z\left(t z^{2}+y z+y w\right)=0
\end{aligned}
$$

que são as equações de definição de $X$ e das equações determinadas pelos menores $3 \times 3$ da matriz

$$
\left[J f_{\lambda}, \omega\right]=\left(\begin{array}{cccc}
-y & -x-\lambda & w & z \\
w & -2 y w-2 y z-t z^{2} & -y^{2}-2 t z y & x-y^{2} \\
2 x-y^{2}+\lambda & -2 y(x+\lambda)-z^{2} & -2 z y-3 t z^{2} & 0 \\
0 & 1 & -1 & 0
\end{array}\right)
$$


Resolvendo este sistema de equações, obtemos as seguintes soluções:

$$
\begin{aligned}
& (0,0,0, \lambda) \\
& \left(-\lambda, \pm \sqrt{-\lambda}, 0, w_{i}^{+}\right) \\
& \left(-\lambda, \pm \sqrt{-\lambda}, 0, w_{i}^{-}\right)
\end{aligned}
$$

onde $i=1,2, w_{i}^{+}, w_{i}^{-}$são soluções das equações

$$
\begin{aligned}
& w^{2} \mp 2(-\lambda) w \pm \lambda \sqrt{-\lambda}=0 \\
& w^{2} \pm 2(-\lambda) w \mp \lambda \sqrt{-\lambda}=0 .
\end{aligned}
$$

As outras soluções satisfazem $x=\frac{y^{2}-\lambda}{2}$ e são obtidas das equações

$$
\begin{aligned}
& (1+3 t) z^{2}+2 y z+y\left(y^{2}+\lambda\right)=0 \\
& w\left(\lambda+y^{2}\right)+2 y\left(y z+t z^{2}\right)=0 \\
& \left(y^{2}+\lambda\right)^{2}+4 z\left(y z+t z^{2}\right)=0
\end{aligned}
$$

É possível mostrar que neste caso, conseguimos mais 3 soluções distintas da forma

$$
\left(\frac{y^{2}-\lambda}{2}, y, \mu_{3} \sqrt[3]{\frac{\left(y^{2}+\lambda\right)\left(y^{2}+\lambda-2 y\right)}{2(1+t)}}, w(y)\right)
$$

onde $w(y)$ é solução da equação

$$
\frac{y^{2}\left(y^{2}+\lambda\right)-y \mu_{3}^{2} \rho(y)(1+t)}{y^{2}+\lambda},
$$

com

$$
\rho(y)=\sqrt[3]{\frac{\left(y^{2}+\lambda\right)\left(y^{2}+\lambda-2 y\right)}{2(1+t)}} .
$$

Logo, $\operatorname{ind}_{P H}(X ; \omega, 0)=8$. Portanto, $\mu(X)=5=\tau(X)-1$.

Suponhamos que para $k-1$, o número de Milnor de $M$ é $2 k+1$. Vamos mostrar que para $k, \mu(X)=\tau(X)-1=2 k+3$. Consideremos a seguinte deformação a 1 - parâmetro de $M$ dada por

$$
M_{t}=\left(\begin{array}{ccc}
z & y & x \\
x & w & y z+y^{k} w+t y^{k-1} w
\end{array}\right),
$$

onde $t \in \mathbb{C}$ e $t \neq 0$. A variedade $X_{t}$ definida pelos menores maximais de $M_{t}$ é singular na origem e nos pontos $\left(0,-t, 0, \pm \sqrt{-t^{3}}\right)$. Logo,

$$
\mu(X)=\mu\left(X_{t}, 0\right)+\mu\left(X_{t}, u_{1}\right)+\mu\left(X_{t}, u_{2}\right)
$$


onde 0 representa a origem em $\mathbb{C}^{4}, u_{i}=\left(0,-t, 0,(-1)^{i} \sqrt{-t^{3}}\right)$.

No ponto $x=y=z=0, y \neq-t$, então $t+y$ é uma unidade em $\mathcal{P}$. Logo,

$$
\begin{aligned}
\left(\begin{array}{ccc}
z & y & x \\
x & w & y z+w y^{k-1}(t+y)
\end{array}\right) & \sim\left(\begin{array}{ccc}
z & y & x /(t+y) \\
x & w & \frac{y z}{t+y}+w y^{k-1}
\end{array}\right) \sim \\
\left(\begin{array}{ccc}
z(t+y) & y & x /(t+y) \\
x & w & y z+w y^{k-1}
\end{array}\right) & \sim\left(\begin{array}{ccc}
z & y & x \\
x & w & y z+w y^{k-1}
\end{array}\right) .
\end{aligned}
$$

Pela hipótese de indução $\mu\left(X_{t}, 0\right)=2 k+1$.

Quando $x=z=0, y=-t$ e $w= \pm \sqrt{-t^{3}}$, a matriz é equivalente à

$$
\left(\begin{array}{ccc}
x & z y+a y^{k}+t w y^{k-1} & w \\
z & x & y
\end{array}\right) .
$$

Calculando o 1- jato de $z y+a y^{k}+t w y^{k-1}$, temos que

$$
\left(\begin{array}{ccc}
x & c y+d z & w \\
z & x & y
\end{array}\right)
$$

com $c, d \neq 0$, que é portanto, equivalente à primeira forma normal cujo o número de Milnor é 1 .

Portanto, $\mu(X)=2 k+1+1+1=2 k+3$.

\section{- $6^{\text {o }}$ Forma Normal:}

Denotamos por $M$ a matriz que representa a $6^{\circ}$ forma normal e consideremos a projeção

$$
\begin{aligned}
p: \quad X & \longrightarrow \mathbb{C} \\
(x, y, z, w) & \longmapsto y .
\end{aligned}
$$

Neste caso, se $X$ é a variedade determinantal definida por $M$, então consideramos a curva determinantal $C=X \cap p^{-1}(0)$ dada por

$$
M=\left(\begin{array}{lll}
w & x & z^{2} \\
0 & z & x
\end{array}\right)
$$

que é a primeira forma normal obtida em [16], com $k=5$. Procedendo de forma análoga ao feito para a segunda forma normal, encontramos uma suavização

$$
M_{\lambda}=\left(\begin{array}{ccc}
w & x+\lambda & z^{2}+\lambda \\
y & z & x
\end{array}\right)
$$


para $M$, onde $\lambda \in \mathbb{C}, \lambda \neq 0$. Se $\omega=d p$, resolvemos o sistema de equações determinado por $f_{\lambda}$ e pelos menores $3 \times 3$ da matriz $\left[J f_{\lambda}, \omega\right], f_{\lambda}$ é a aplicação determinada pelos menores $2 \times 2$ de $M_{\lambda}$, obtemos o $\operatorname{ind}_{P H}(\omega ; X, 0)=9$. Uma vez que $\mu(C)=3$, segue que $\mu(X)=6$.

- $7^{\mathrm{o}}, 9^{\mathrm{o}}, 11^{\mathrm{o}}$ e $12^{\mathrm{o}}$ Formas Normais:

Sejam $p_{i}: \mathbb{C}^{4} \longrightarrow \mathbb{C}, i=1,2$, projeções lineares dadas por $p_{1}(x, y, z, w)=y$ para a sétima forma normal e $p_{2}(x, y, z, w)=w$ as demais. Em todos os casos, a curva determinantal simples $C=X \cap p_{i}^{-1}(0)$ é definida pela matriz

$$
M=\left(\begin{array}{lll}
w & x & z^{2} \\
0 & z & x
\end{array}\right),
$$

que é a primeira forma normal obtida em [16], com $k=5$. Usando procedimento análogo ao feito para a primeira forma normal, mostramos que nestes casos, $\mu(X)=\tau(X)-1$.

Observação 6.3.1. Seja $(X, 0)$ um germe de superfície determinantal, quase- homogênea de codimensão 2. Conjecturamos, que a fórmula $\tau(X)=\mu(X)+1$, seja verdadeira para tais variedades. 


\section{Referências Bibliográficas}

[1] E. Arbarello, M. Cornalba, P. A. Griffiths, J. Harris, Geometry of Algebraic Curves, v.1 Grundlehren der Mathematischen Wissenschaften [Fundamental Principles of Mathematical Sciences], Springer- Verlang, New York, (1985).

[2] V. I. Arnold, Matrices depending on parameters, Russian Math. Surveys 26 (1971), no. 2, 29-46.

[3] J. W. Bruce, Families of symmetric matrices, Moscow Math. J., 3, no 2, (2003), 335-360.

[4] J. W. Bruce, R. M. Roberts, Critical points of functions on analytic varieties, Topology, 27, no 1, (1988), 57-90.

[5] J. W. Bruce and F. TARi, On families of square matrices, Proc. London Math. Soc. (3), 89, (2004),738-762.

[6] R.-O. G. Buchweitz And G.- M. Greuel, The Milnor Number and Deformations of Complex Curve Singularities, Inventiones Mathematicae, 58, (1980), 241- 281.

[7] W. Bruns e U. Vetter, Determinantal Rings, Springer- Verlang, New York, (1998).

[8] W. Bruns e J. Herzog, Cohen-Macaulay Rings, Revised edition, Cambridge University Press, New York, (1998).

[9] J. Damon, Deformations of sections of singularities and Gorenstein surface singularities, Amer. J. Math., 109 (1987),no. 4, 695-721. MR 88i:58013.

[10] J. DAmon, The unfolding and determinancy theorems for subgroups of $\mathcal{A}$ and $\mathcal{K}$, Memoirs of the American Mathematical Society, Providence RI, 1984.

[11] J. A. Eagon, D. G. Northcott D. G., Ideals defined by matrices and a certain complex associated with them, Proc. Roy. Soc. London Ser. A ; 1811, 269,(1962), 188-204. 
[12] J. A. EAGON, Ideals generated by subdeterminants of a matrix, Thesis, University of Chicago,(1961).

[13] W. Ebeling E S. M. Gusein-Zade, On indices of 1-forms on determinantal singularities, Singularities and Applications, 267, 119- 131, (2009).

[14] W. Ebeling e S. M. Gusein-Zade, Radial Index and Euler Obstruction of a 1-form on singular varieties, Geometriae Dedicata, 113, 231- 241 (2005).

[15] D. Eisenbud, Commutative algebra with a view toward algebraic geometry, Graduate text in mathematics; 150, Spriger-Verlang New York, (1995).

[16] A. FrüHbis-KrüGer, Classification of Simple Space Curves Singularities, Comm. in Alg., 27 (8), (1999), 3993-4013.

[17] A. Frühbis-Krüger, Moduli Spaces for Space Curve Singularities, Universität Kaiserslautern, Ph. D, (2000).

[18] A. Frühbis-Krüger, A. Neumer, Simple Cohen-Macaulay Codimension 2 Singularities, arXiv:0808.2439v2, (2008).

[19] T. Gaffney, Polar multiplicities and equisingularity of map germs, Topology, 32,(1993), 185- 223.

[20] T. Gaffney, Properties of finitely determined germs, Brandeis University, Ph. D., 107, (1976), Mathematics.

[21] T. Gaffney, N. Grulha JR., The multiplicity polar theorem, collections of 1-forms and Chern numbers, em preparação.

[22] C. G. Gibson, Singular points of smooth mappings, Research notes in mathematics, 25, London; San Francisco : Pitman (1979).

[23] C. G. Gibson, K. Wirthmuller, A. A. Du Plessis e E. J. Looijenga, Topological Stability of Smooth Mappings, Lectures Notes in Mathematics, $\mathbf{5 5 2}$.

[24] G. M. Greuel, J. Steenbrink, On the Topology of Smoothable Singularities, Proceedings of Symposia in Pure Mathematics, 40, (1983), Part 1, 535- 545.

[25] G. M. Greuel, Der Gauss- Manin Zusammenhang isolierter Singularitäten von vollständigen Durchschnitten, Math. Ann., 214, (1975), 235- 266. 
[26] G.-M. Greuel, B. Martin e G. Pfister, Numerische Charakterisierung quasihomogener Gorenstein-Kurvensingularitäten, Math. Nachr., 124, (1985), 123-131.

[27] M. Hoschter, J. A. EAgon, Cohen- Macaulay rings, invariant theory, and generic perfection of determinantal loci, Amer. J. Math. , 93,(1971), 1020-1058.

[28] G. J. Hashinger, Families of skew-simmetric matrices, University of Liverpool Thesis, 2001.

[29] H. A. HАмm, Lokale topologische Eigenschaften komplexer Räume, Math. Ann., 191, (1972), 235- 252 .

[30] R. Hartshorn, Algebraic Geometry, Graduate Texts on Mathematics, 52, SpringerVerlag, New York, Heidelberg Berlin.

[31] M. Hochester, J. A. Eagon, A class of perfect determinantal ideals, Bull. Amer. Math. Soc., 76, No 5 (1970), 1026-1029.

[32] M. Hochester, J. A. EAgon, Cohen-Macaulay rings, invariant theory, and the generic perfection of determinantal locci, Amer. Math. Soc., 93, No 5 (1971), 1020-1058.

[33] T. C. KuO, On $\mathcal{C}^{0}$-sufficiency of jets of potential functions, Topology, 8,(1969), 167-171.

[34] D. Laksov, Deformation of determinantal schemes, Compositio Mathematics; 30, (1975), No. 3, 273-292.

[35] A. Markoe, A Characterization of Normal Analytic Spaces by the Homological Codimension of the Structure Sheaf, Pacific Journal of Matehmatics, v. 52, no. 2, (1974).

[36] W. J. Milnor, Singular Points of Complex Hypersurfaces, Annals of Mathematics Studies, 25, New Jersey, (1968).

[37] W. J. Milnor, Morse Theory - Based on lecture notes by M. Spivak and R. Wells, Annals of Mathematics Studies, 51, New Jersey, (1963).

[38] M. A. S. RuAs, M. J. SAIA, $\mathcal{C}^{l}$ - determinacy of weighted homogeneous germs, Hokkaido Mathematical Journal 26, (1997), 89-99.

[39] M. A. S. Ruas E J. N. Tomazella, Topological triviality of families of functions on analytic varieties, Nagoya Math. J. 175 (2004), 39-50. 
[40] M. A. S. Ruas e J. N. Tomazella, An infinitesimal criterion for topological triviality of families of sections of analytic varieties, Advanced Studies in Pure Mathematcs (Amsterdam), v. 43, pág. 421-436, 2006.

[41] M. Schaps, Nonsingular Deformations of a Determinantal Scheme, Pacific Journal of Mathematics, 65, No 1, (1976), pág. 209-215.

[42] M. Schaps, Deformations of Cohen-Macaulay Schemes of Codimension 2 and NonSingular Deformations of Space Curves, American Journal of Mathematics, 99, No 4, (1977), pág. 669-685.

[43] M. Schaps, Versal Determinantal Deformations, Pacific Journal of Mathematics, 107, No 1, (1983), pág. 213-221.

[44] K. SAIto, Quasihomogene Isolierte Singularitäten von Hyperflächen, Invent. Math., 14, (1971), 123-142.

[45] J. Stevens, Deformation of Singularities, Lecture Notes in Mathematics; 1811, SpringerVerlang Berlin Heidelberg New York, (2003).

[46] TuURina, G. N., Absolute isolatedness of rational singularities and triple rational points, Func. Anal. Appl., 2, 324- 333,(1968).

[47] B. Tessien, Variéties polaires 2: Multiplicités polaires, sections planes, et conditions de Whitney. Actes de la conference de géometrie algébrique à la Ràbida, Springer Lecture Notes, \# 961, pág. 314- 491 (1981).

[48] LÊ D. T., B. Tessier, Variétés polaires locales et classes de Chern des variétés singulières, Ann. of Math., (1992), 83-100.

[49] LÊ D. T. , Complex Analytic Functions With Isoleted Singularities, J. Algebraic Geometry, 2, 114, (1981), No 3, 457- 491.

[50] LÊ D. T. , Calculation of Milnor Number of an Isolated Singularity of Complete Intersection, Funct. Anal. and Appl., 8, (1974), 127-131.

[51] J. WAHL, Smoothings of normal surface singularities, Topology, 20, (1981), 219- 246.

[52] C. T. WALl, Finite determinacy of smooth map-germs, Bull. London Math. Soc. 13 (1981), no 6, 481-539. MR 83i:58020. 
[53] H. Whitney, Tangents to an Analytic Variety, Ann. Math., 93, No 5 (1971), 1020-1058.

[54] J. WAHL, A characterization of quasi-homogeneous Gorenstein surface singularities, Compositio Math., 55, (1985), 269-288. 